\title{
$\beta-N$-Acetylhexosaminidases for Carbohydrate Synthesis via Trans-Glycosylation
}

\author{
Jan Muschiol ${ }^{\circ}$, Marlene Vuillemin, Anne S. Meyer * and Birgitte Zeuner ${ }^{(\mathbb{C}}$ \\ Department of Biotechnology and Biomedicine (DTU Bioengineering), Technical University of Denmark, Søltofts \\ Plads 221, 2800 Kongens Lyngby, Denmark; jmus@dtu.dk (J.M.); mavu@dtu.dk (M.V.); bzeu@dtu.dk (B.Z.) \\ * Correspondence: asme@dtu.dk
}

Received: 10 March 2020; Accepted: 26 March 2020; Published: 29 March 2020

\begin{abstract}
N$-acetylhexosaminidases (EC 3.2.1.52) are retaining hydrolases of glycoside hydrolase family 20 (GH20). These enzymes catalyze hydrolysis of terminal, non-reducing $N$-acetylhexosamine residues, notably $N$-acetylglucosamine or $N$-acetylgalactosamine, in $N$-acetyl- $\beta$-D-hexosaminides. In nature, bacterial $\beta-N$-acetylhexosaminidases are mainly involved in cell wall peptidoglycan synthesis, analogously, fungal $\beta-N$-acetylhexosaminidases act on cell wall chitin. The enzymes work via a distinct substrate-assisted mechanism that utilizes the 2-acetamido group as nucleophile. Curiously, the $\beta-N$-acetylhexosaminidases possess an inherent trans-glycosylation ability which is potentially useful for biocatalytic synthesis of functional carbohydrates, including biomimetic synthesis of human milk oligosaccharides and other glycan-functionalized compounds. In this review, we summarize the reaction engineering approaches (donor substrate activation, additives, and reaction conditions) that have proven useful for enhancing trans-glycosylation activity of GH20 $\beta$ - $N$-acetylhexosaminidases. We provide comprehensive overviews of reported synthesis reactions with GH20 enzymes, including tables that list the specific enzyme used, donor and acceptor substrates, reaction conditions, and details of the products and yields obtained. We also describe the active site traits and mutations that appear to favor trans-glycosylation activity of GH20 $\beta-N$-acetylhexosaminidases. Finally, we discuss novel protein engineering strategies and suggest potential "hotspots" for mutations to promote trans-glycosylation activity in GH20 for efficient synthesis of specific functional carbohydrates and other glyco-engineered products.
\end{abstract}

Keywords: glycoside hydrolase family 20; GH20; lacto- $N$-biosidase; human milk oligosaccharides; reverse hydrolysis; enzyme engineering; reaction engineering; regioselectivity; $\mathrm{N}$-acetylglucosamine; oxazoline

\section{Introduction}

$\mathrm{N}$-acetylhexosamines are important constituents in several biological and biochemically significant structures. The most abundant representative is the 2-acetamido-2-deoxy derivative of glucose, namely $\mathrm{N}$-acetylglucosamine (GlcNAc), which is the main constituent of chitin-the second most abundant biopolymer on earth. Chitin is found in the exoskeletons of arthropods (e.g., shrimp, crabs, and insects) and in fungal cell walls [1,2]. Furthermore, both GlcNAc and its epimer N-acetylgalactosamine (GalNAc) are essential constituents of protein glycosylation structures in eukaryotes as they form part of the glycan core structures.

$\beta-N$-acetylhexosaminidases (EC 3.2.1.52) of glycoside hydrolase family 20 (GH20) catalyze the hydrolytic removal of $N$-acetylhexosamines from the non-reducing end of $\mathrm{N}$-acetyl- $\beta$-D-hexosaminides and may act on either $\mathrm{N}$-acetylglucosides or $\mathrm{N}$-acetylgalactosides (including chains of mixed glucosides as long as either GlcNAc or GalNAc is in the non-reducing end) [3]. 
Until recently, the $\beta-N$-acetylhexosaminidases were studied mainly due to their importance in the pathogenesis of the hereditary neuro-metabolic disorders Tay-Sachs and Sandhoff diseases, that both result from mutations in hexosaminidases $[3,4]$. The microbial $\beta$ - $N$-acetylhexosaminidases are mainly involved in cell wall synthesis and growth, i.e., in bacteria they act on the peptidoglycan, and in fungi on the chitin. The microbial $\beta-N$-acetylhexosaminidases have lately received attention due to their potential usefulness in new biosynthetic processes via their ability to catalyze reverse hydrolytic and trans-glycosylation reactions. Such reactions are of significance in novel industrial renewable carbon upcycling and biomimetic glycan synthesis processes.

GlcNAc is probably the hexosamine of the biggest industrial interest since it can be isolated from large industrial waste streams, e.g., from shrimp and crab waste (because chitin is a main constituent of the shrimp and crab shells) and either serve as a renewable carbon and nitrogen source for bio-ethanol production [5] or be used as a constituent for higher value functional applications, e.g., in medicine, high-value food ingredients, or cosmetics [6]. Prominent examples of such high value GlcNAc-containing food-ingredient products are the human milk oligosaccharides (HMOs, Figure 1) [7,8]. Apart from the simplest HMOs such as $2^{\prime}-$ and 3-fucosyllactose as well as $3^{\prime}$ - and $6^{\prime}$-sialyllactose, all HMOs contain at least one GlcNAc moiety [9]. Because of their beneficial effects for infant development, health, and nutrition [10] and the fact that infant formulae based on cow's milk lack HMOs, several companies have recently started specific research for production of HMOs (e.g., Glycom A/S, Denmark; Abbott Laboratories, USA; Jennewein Biotechnologie GmbH, Germany). In 2016, the first infant formula with a biosynthetically produced GlcNAc-containing HMO (Lacto- $N$-neotetraose (LNnT, Figure 1) produced by Glycom A/S) was successfully brought to the market by Nestle [11].

Other possible GlcNAc-containing products are non-reducing oligosaccharides as found in some antibiotics (e.g., tunicamycin [12] or orthosomycins [13]), chitooligomers, for which antioxidative [14,15] and antiangiogenic effects [16] have been demonstrated, and branched glycans/oligosaccharides (e.g., HMOs) (Figure 1). Alkylated GlcNAc moieties are part of molecules that can be used as glycosurfactants $[17,18]$ or bivalent lectin ligands $[19,20]$, GlcNAc-modified drugs and vitamins have a higher solubility in water [21,22], and GlcNAc-modified amino acids and glyco-engineered proteins are important for optimal bioactivity $[23,24]$.

In summary, the portfolio of GlcNAc-containing interesting molecules is broad and it would therefore also require a large set of chemical methods and catalysts to synthesize all of them. Especially the chemical synthesis of glycan suffers significantly from laborious protection and de-protection steps. As a result, biocatalytic synthesis involving reverse hydrolytic or trans-glycosylation reactions of GH20 $\beta-N$-acetylhexosaminidases have recently gained increased attention. 

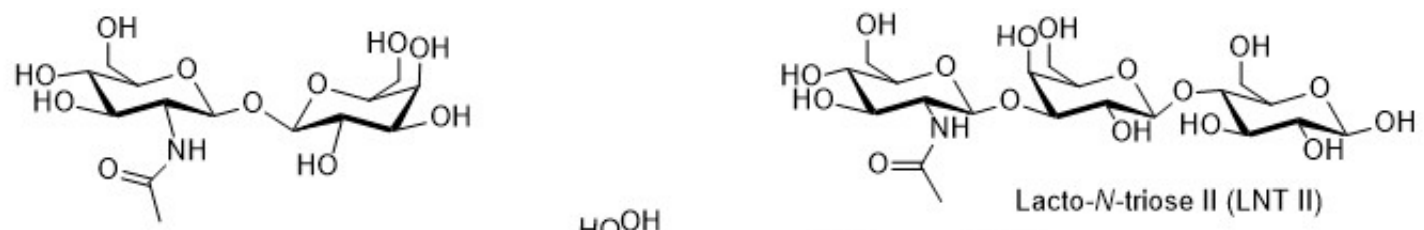

Non-reducing oligosaccharides
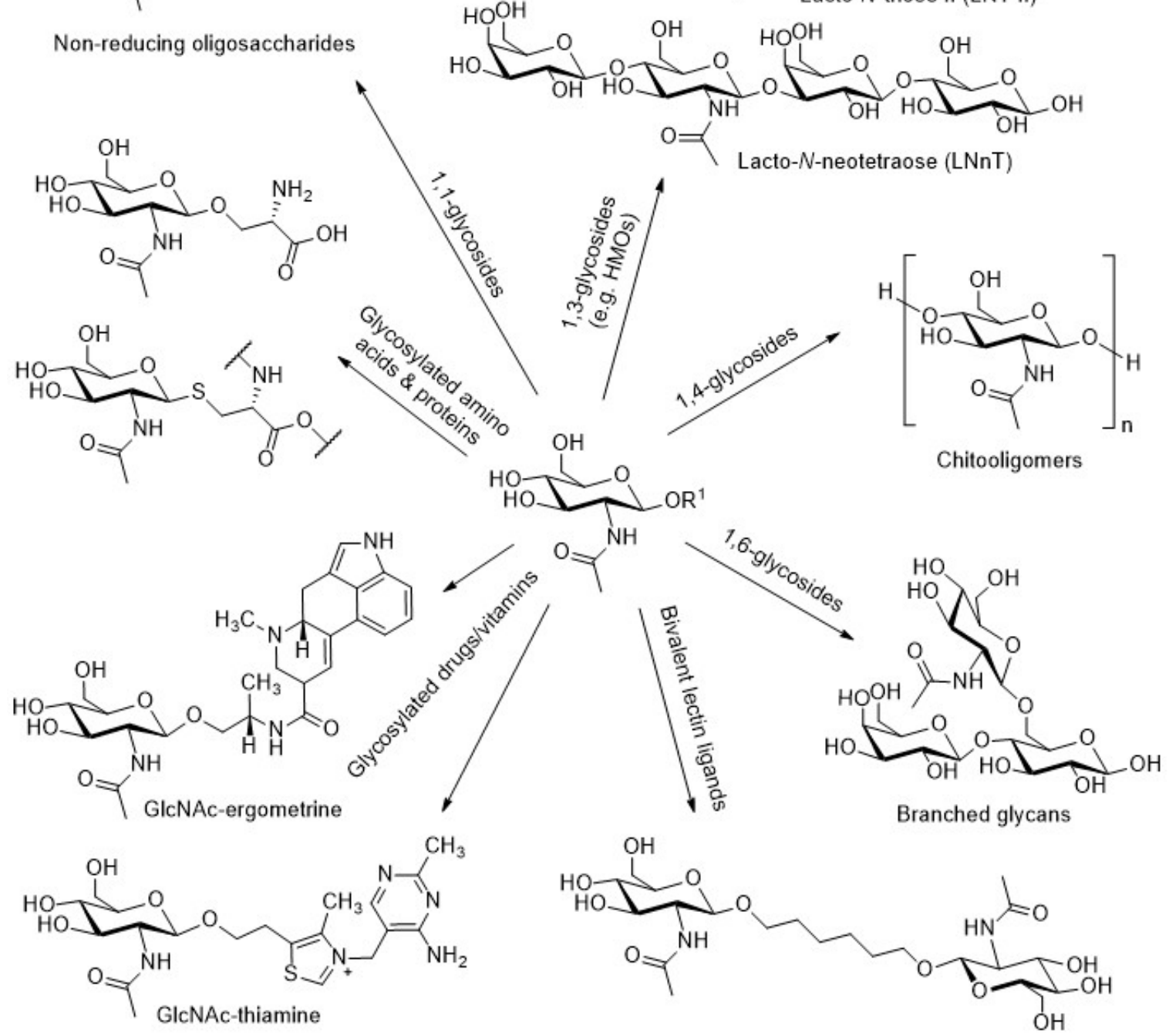

Figure 1. Products from GH20-catalyzed trans-glycosylation reactions.

\section{GH20 $\beta-N$-Acetylhexosaminidases}

High-value GlcNAc-containing oligosaccharides or glycosylated molecules can be synthesized using GH20 $\beta$-N-acetylhexosaminidases. These enzymes can be found throughout the whole tree of life. However, in the CAZy database [25] there are 16 times more bacterial GH20 $\beta$ - $N$-acetylhexos-aminidase sequences than from any other domain of life. In contrast, there are significantly more studies using fungal $\beta-N$-acetyl hexosaminidases than of any other origin, which is due to the eminent contribution to this field by Vladimír Křen and colleagues.

Furthermore, GH20 $\beta-N$-acetylhexosaminidases are usually well-expressed in a recombinant host (either cytosolic or secreted) or can be easily purified from wild-type cultivations since these enzymes are often secreted by their natural host organism. This facilitates simple purification of the enzymes for biocatalytic purposes, which was applied in $>95 \%$ of the literature studied for this article.

Mechanistically, enzymes from family GH20 are special. In contrast to most other glycoside hydrolases (GHs), the hydrolytic cleavage of the glycosidic linkage to the GlcNAc residues is catalyzed via a so-called substrate-assisted mechanism (Figure 2) [26-30]. In this case, the reaction intermediate is not bound to the enzyme as is the case for the classical Koshland mechanism [31]. The reactive 
intermediate is created with assistance of the substrate's 2-acetamido group, which participates in formation of a glucoxazolinium ion intermediate upon cleavage of the glycosidic bond (Figure 2). Whether the intermediate in the GH20 catalysis is actually the oxazolinium ion as proposed for GH84 $\mathrm{O}-\mathrm{GlcNA}$ ases [32] or the uncharged glucoxazoline (Glc-oxa) as proposed for GH18 chitinases [33] is not fully clarified yet. However, it is tempting to speculate that due to the close resemblance of the catalytic mechanisms and motifs of enzymes from families GH20 and GH84 (adjacent Asp-Glu pair), and their proposed substrate-assisted catalytic mechanism, the formation of a glucoxazolinium ion is likely. Nucleophilic attack of water then leads to release of GlcNAc as a reaction product (hydrolysis, Figure 2: $R^{1} \neq H, R^{2}=H$ ). During this attack the water molecule is stabilized in the active site by a conserved Tyr residue [34]. However, it is a general feature of many retaining GHs that other nucleophiles such as carbohydrates or alcohols are also accepted, which then leads to a glycosylated product (trans-glycosylation, Figure $2: \mathrm{R}^{1} \neq \mathrm{H}, \mathrm{R}^{2} \neq \mathrm{H}$ ) [35]. Most of the CAZymes acting on GlcNAc residues follow this mechanism (e.g., chitinases (GH18), O-GlcNAcases (GH84), endo- $\beta$ - $N$-acetylglucosaminidases (GH85)) [3,36-38]. Only hexosaminidases from family GH3 [39] and the recently discovered GH136 lacto-N-biosidase LnbX from Bifidobacterium longum subsp. longum [40] were shown to catalyze cleavage of their substrates utilizing the classical double-displacement mechanism involving an enzyme-coupled intermediate.

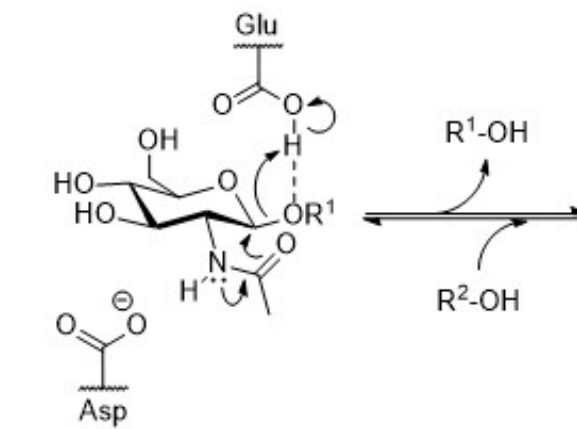

GIcNAc: $\mathrm{R}^{1}=\mathrm{H}$

activated GIcNAc: $\mathrm{R}^{1}$ = carbohydrate, alkyl

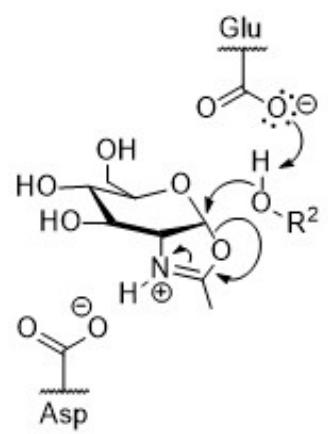

Glucoxazolinium ion

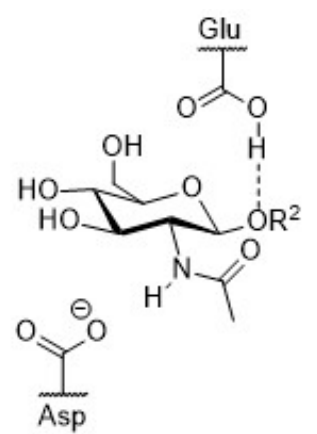

Hydrolysis: $\mathrm{R}^{2}=\mathrm{H}$

Transglycosylation: $\mathrm{R}^{2}$ = carbohydrate, alkyl

Figure 2. Proposed substrate-assisted mechanism of GH20 $\beta-N$-acetylhexosaminidases [29]. Indication of the intermediate as a Glc-oxazolinium ion is based on recent computational studies on a GH84 O-GlcNAcase probably utilizing the same mechanism [32].

In nature, glycans, oligo- and polysaccharides as well as other glycosylated molecules are synthesized by glycosyltransferases (GTs), which strictly require a nucleotide-activated derivative as substrate. Therefore, these enzymes are not very attractive for biocatalytic reactions, because the prices for nucleotide-activated sugars are exceptionally high $(1,220,000 € / \mathrm{kg}(795,000 € / \mathrm{mol})$ for UDP-GlcNAc at Carbosynth) [41]. Furthermore, functional expression of GTs can be challenging. In contrast, GHs with trans-glycosylase activity are more attractive for large-scale in vitro synthesis of such valuable products. GHs have a broader substrate acceptance and can use non-natural activated carbohydrates or natural disaccharides as donor molecules. However, challenges are also encountered with GH trans-glycosylases. Firstly, the regioselectivity of GH-catalyzed trans-glycosylation reactions can be rather low, which leads to a mixture of the desired product and undesired side products. Secondly, GH trans-glycosylases are in fact hydrolytic enzymes or are derived from such. They are usually still able to hydrolyze both the substrate and the desired product (secondary hydrolysis) [35,42]. However, as outlined in the following, several strategies may be employed to increase trans-glycosylation activity and/or diminish hydrolytic activity in general. 


\section{Increased Trans-Glycosylation Activity by Reaction Engineering}

Since synthesis using GHs can be compromised by secondary hydrolysis and low regioselectivity, a number of reaction engineering strategies, notably including various donor molecule activation approaches, have been reported to help increase yields of GH20-catalyzed trans-glycosylation reactions. Surprisingly, immobilization or continuous product removal approaches, which have been successfully applied for other GH-catalyzed trans-glycosylation reactions [42], have not yet been used to optimize GH20 catalyzed trans-glycosylation reactions.

\subsection{Reverse Hydrolysis VS. Trans-Glycosylation}

In general, GH-catalyzed synthesis of oligosaccharides or other glycosylated products can be carried out in two different modes of reaction: reverse hydrolysis or trans-glycosylation.

In a reverse hydrolysis reaction, the GH catalyzes a condensation reaction of two carbohydrates (where the activated donor cannot be distinguished among the two reactants) to yield specific disaccharides (Table 1, Figure 2: $R^{1}=H$ and $R^{2} \neq H$ ). The formation of glycosylated amino acids and alkyl glycosides by enzyme-catalyzed reverse hydrolysis have also been described (Table 1). To the best of our knowledge, the term reverse hydrolysis was first used in a review from 1986 [43] and later in the context of glucose-disaccharide formation by $\beta$-glucosidase from almond [44], where the authors demonstrated that a high substrate load is required and that a high reaction temperature favors product formation. Ten years later, the first reports on GH20-catalyzed reactions involving the hexosaminidases from Bacillus circulans (BcHex) and Aspergillus oryzae (AoHex) via reverse hydrolysis setup were published $[45,46]$. Clear disadvantages of such reverse hydrolysis reactions are the long reaction times (72-360 h, Table 1) and the low yields, which rarely exceed 15\% isolated yield [23] though higher yields (up to $46 \%$, Table 1) have been reported for non-isolated product outcomes [47].

In contrast, significantly higher yields can be achieved using shorter reaction times in trans-glycosylation reactions (Tables 2-9), where a carbohydrate moiety is transferred from an activated donor molecule to an acceptor molecule containing a free hydroxyl group. The presence of a good leaving group on $\mathrm{C} 1$ of the activated donor molecule and a high concentration of acceptor drive the reaction. This approach was used in the first trans-glycosylation reaction reported for a GH20 enzyme by Mega et al. in 1972 [48]. In that pioneering study, the trans-glycosylation ability of the hexosaminidase from Aspergillus oryzae ( $\mathrm{AoHex}$ ) was demonstrated in an auto-condensation reaction of phenyl-activated GlcNAc (Figure 3: GlcNAc-Ph), which mainly led to the formation of GlcNAc- $\beta-1,4-G l c N A c-\beta-P h$ (Table 2). The auto-condensation reaction is a special case of trans-glycosylation, since it involves only one substrate, which acts as both donor and acceptor. 
Table 1. Synthetic approaches using GH20 enzymes in reverse hydrolysis reactions. New bonds are highlighted in bold.

\begin{tabular}{|c|c|c|c|c|c|c|c|c|c|}
\hline Enzyme (Organism) & $\begin{array}{l}\text { Acceptor } \\
(c[\mathrm{mM}])\end{array}$ & $\begin{array}{l}\text { Donor } \\
(c[\mathrm{mM}])\end{array}$ & $\begin{array}{l}\text { A:D } \\
\text { Ratio }\end{array}$ & Product(s) & $\mathrm{pH}$ & $T\left[{ }^{\circ} \mathrm{C}\right]$ & $t[\mathrm{~h}]$ & $\begin{array}{l}\text { Yield } \\
\text { [\% }^{1}\end{array}$ & Ref. \\
\hline \multirow[t]{2}{*}{$\begin{array}{l}\text { AnHex (Aspergillus } \\
\text { niger CCIM K2) }\end{array}$} & \multicolumn{2}{|c|}{ GalNAc $(1500)^{2}$} & $1: 1$ & $\begin{array}{l}\text { GalNAc- } \alpha-1,3-G a l N A c \text { (I), } \\
\text { GalNAc- } \alpha-1,6-G a l N A c \text { (II) }\end{array}$ & 3.6 & 35 & 120 & $15(\mathrm{I}+\mathrm{II})^{3}$ & [23] \\
\hline & $t$-Boc-Ser, $t$-Boc-Thr (1130) & $\alpha$-GalNAc (1130) & $1: 1$ & $\begin{array}{l}\text { GalNAc- } \alpha-O-S e r(\mathrm{I}) \\
\text { GalNAc- } \alpha-O \text {-Thr (II) }\end{array}$ & 4.8 & 35 & 168 & 7.4 (I), 3.6 (II) ${ }^{3,4}$ & {$[23]$} \\
\hline $\begin{array}{l}\text { AoHex (Aspergillus } \\
\text { oryzae) }\end{array}$ & $\begin{array}{l}\text { GlcNAc- } \beta-N-A c(1115) \\
\text { GlcNAc- } \beta-N-\operatorname{Pr}(754)\end{array}$ & GlcNAc (113/75) & 10:1 & $\begin{array}{l}\text { GlcNAc- } \beta-1,6-G l c N A c-\beta-N-A c \text { (I), } \\
\text { GlcNAc- } \beta-1,6-G l c N A c-\beta-N-P r \text { (II) }\end{array}$ & 5.0 & 37 & 120,168 & $13(\mathrm{I}), 8$ (II) $)^{3,5}$ & [49] \\
\hline $\begin{array}{c}\text { AoHex (Aspergillus } \\
\text { oryzae CCF 1066) }\end{array}$ & \multicolumn{2}{|c|}{$\operatorname{GlcNAc}(1500)^{2}$} & $1: 1$ & GlcNAc- $\beta-1,6-G l c N A c$ & 4.9 & 39 & 144 & $1.9-14.5^{3,6}$ & [46] \\
\hline & Alcohols and diols & GlcNAc & n.a. & Alkyl glycosides ${ }^{7}(\beta-O)$ & 4.2 & 37 & 72 & n.a. ${ }^{6}$ & [46] \\
\hline $\begin{array}{l}\text { AoHex (Aspergillus } \\
\text { oryzae RIB40) }\end{array}$ & Lac (1218) & GlcNAc (565) & $2: 1$ & $\begin{array}{c}\text { LNT II }(\beta-1,3)(\mathrm{I}) \\
\text { GlcNAc- } \beta-1,6-\text { Lac (II) }\end{array}$ & 6.0 & 45 & 96 & $0.36(\mathrm{I}), 0.72(\mathrm{II})^{3}$ & {$[50]$} \\
\hline $\begin{array}{l}\text { LnbB (Bifidobacterium } \\
\text { bifidum JCM1254) }\end{array}$ & Lac (1000) & LNB (100) & 10:1 & $\begin{array}{c}\text { LNT }(\beta-1,3) \text {, one unidentified } \\
\text { regioisomer } 8\end{array}$ & 4.5 & 40 & n.a. & n.d. ${ }^{9}$ & {$[51]$} \\
\hline $\begin{array}{l}\text { BcHex (Bacillus } \\
\text { circulans) }\end{array}$ & Man (9436) & GlcNAc (2260) & $4: 1$ & $\begin{array}{l}\text { GlcNAc- } \boldsymbol{\beta}-\mathbf{1}, \mathbf{2}-\operatorname{Man}(\mathrm{I}) \\
\text { GlcNAc- } \boldsymbol{\beta}-\mathbf{1 , 6}-\mathrm{Man} \text { (II) }\end{array}$ & 5.0 & 37 & 360 & $0.3(\mathrm{I}), 2(\mathrm{II})^{3}$ & {$[45]$} \\
\hline $\begin{array}{l}\text { PfHex (Penicillium } \\
\text { funiculosus CCF 1994) }\end{array}$ & \multicolumn{2}{|c|}{$\operatorname{GlcNAc}(1031)^{2}$} & $1: 1$ & $\begin{array}{l}\text { GlcNAc- } \boldsymbol{\beta}-\mathbf{1}, \mathbf{3}-G l c N A c \text { (I), } \\
\text { GlcNAc- } \boldsymbol{\beta}-\mathbf{1 , 4}-\text { GlcNAc (II), } \\
\text { GlcNAc- } \boldsymbol{\beta} \mathbf{- 1 , 6 - G l c N A c ~ ( I I I ) ~}\end{array}$ & 5.0 & 37 & 192 & $\begin{array}{l}3.8 \text { (I), } 1.7(\mathrm{II}), 10 \\
\quad(\mathrm{III}){ }^{3,5}\end{array}$ & [52] \\
\hline $\begin{array}{l}\text { PgHex (Phoma } \\
\text { glomerata) }\end{array}$ & $\mathrm{MeOH}(4944)$ & $\begin{array}{l}\text { GlcNAc, GalNAc } \\
\text { (90 each) }\end{array}$ & $55: 1$ & $\begin{array}{l}\text { GlcNAc- } \beta-O-M e(I) \\
\text { GalNAc- } \beta-O-M e(I I)\end{array}$ & 7.4 & 37 & 168 & 37.8 (I), 46 (II) ${ }^{9}$ & [47] \\
\hline
\end{tabular}

${ }^{1}$ donor based; ${ }^{2}$ auto-condensation reaction; ${ }^{3}$ isolated yield(s); ${ }^{4}$ isolated as de-protected conjugates; ${ }^{5}$ isolated as peracetylated derivates; ${ }^{6}$ depended on water activity by addition of 0.12-1.17 M $\left(\mathrm{NH}_{4}\right)_{2} \mathrm{SO}_{4}$ or $0.5 \mathrm{M} \mathrm{LiCl} ;{ }^{7}$ product formation only with benzyl alcohol; ${ }^{8}$ probably LNB- $\beta-1,6-\mathrm{Lac} ;{ }^{9}$ product(s) not isolated.

Table 2. Auto-condensation reactions of carbohydrate substrates catalyzed by GH20 $\beta$-N-acetylhexosaminidases. New bonds are highlighted in bold.

\begin{tabular}{|c|c|c|c|c|c|c|c|}
\hline Enzyme (Organism) & $\begin{array}{l}\text { Substrate } \\
(c[\mathrm{mM}])\end{array}$ & Product(s) & $\mathrm{pH}$ & $T\left[{ }^{\circ} \mathrm{C}\right]$ & $t[\mathrm{~h}]$ & $\begin{array}{l}\text { Yield } \\
{[\%]^{1}}\end{array}$ & Ref \\
\hline AoHex (A. oryzae) & pNP-GlcNAc (34) & GlcNAc- $\beta-1,3-G l c N A c-\beta-O-p N P$ & 7.0 & 50 & n.a. & $5.5^{2}$ & [53] \\
\hline 3 & pNP-GlcNAc (24) & $\begin{array}{l}\text { GlcNAc- } \beta-1,4-G l c N A c-\beta-O-p N P(I), \\
\text { GlcNAc- } \beta-1,6-G l c N A c-\beta-O-p N P \text { (II) }\end{array}$ & 6.0 & 35 & 6 & 8 (I), 1.5 (II) ${ }^{2}$ & [54] \\
\hline 3 & $(\mathrm{GlcNAc})_{2}(864)$ & $(\mathrm{GlcNAc})_{3}(\mathrm{I}),(\mathrm{GlcNAc})_{4}$ (II) $($ all $\beta-\mathbf{1}, \mathbf{4})$ & 6.5 & 30 & 25 & $23.3(\mathrm{I}), 7.5$ (II) ${ }^{2}$ & {$[55]$} \\
\hline 3 & pNP-GlcNAc (112) ${ }^{16}$ & $\begin{array}{l}\text { GlcNAc- } \beta-1,4-G l c N A c-\beta-O-p N P(I) \\
\text { GlcNAc- } \beta-1,6-G l c N A c-\beta-O-p N P \text { (II) }\end{array}$ & 5.5 & 37 & 2.5 & $22.2(\mathrm{I}), 3.8$ (II) 2,4 & {$[56]$} \\
\hline 3 & $\begin{array}{c}(\mathrm{GlcNAc})_{4}(161),(\mathrm{GlcNAc})_{3} \\
(122)\end{array}$ & $\left(\mathrm{GlcNAc}_{4}(\mathrm{I}),(\mathrm{GlcNAc})_{5}(\mathrm{II}),(\mathrm{GlcNAc})_{6}(\mathrm{III})(\right.$ all $\boldsymbol{\beta}-\mathbf{1 , 4})$ & 6.5 & 30 & 54,52 & $\begin{array}{l}16 \text { (II) } 2,5,9.2 \text { (III) } 2,5 ; 13.5 \text { (I) } \\
2,6,12.7 \text { (II) } 2,6,6.1 \text { (III) }{ }^{2,6}\end{array}$ & [57] \\
\hline
\end{tabular}


Table 2. Cont.

\begin{tabular}{|c|c|c|c|c|c|c|c|}
\hline Enzyme (Organism) & $\begin{array}{l}\text { Substrate } \\
(c[\mathrm{mM}])\end{array}$ & Product(s) & $\mathrm{pH}$ & $T\left[{ }^{\circ} \mathrm{C}\right]$ & $t[\mathrm{~h}]$ & $\begin{array}{l}\text { Yield } \\
{[\%]^{1}}\end{array}$ & Ref \\
\hline 7 & GlcNAc- $\beta$-O-Ph (38) & 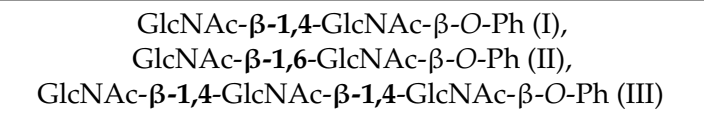 & 6.0 & 37 & 22 & 2.4 (I), 0.2 (II), 0.2 (III) ${ }^{2}$ & {$[48]$} \\
\hline 7 & $\begin{array}{l}\text { 3-O-Me-GlcNAc- } \beta-O-P h \\
\text { 6-O-Me-GlcNAc- } \beta \text {-O-Ph, } \\
\text { GalNAc- } \beta \text {-O-Ph (each 33) }\end{array}$ & $\begin{array}{l}\text { 3-O-Me-GlcNAc- } \beta-\mathbf{1}, \mathbf{4}-3-O-M e-G l c N A c-\beta-O-P h(I), \\
\text { 3-O-Me-GlcNAc- } \beta-\mathbf{1} \text { 6-3-O-Me-GlcNAc- } \beta-O-P h \text { (II), } \\
\text { 6-O-Me-GlcNAc- } \beta-\mathbf{1}, \mathbf{4}-6-O-M e-G l c N A c-\beta-O-P h \text { (III), } \\
\text { GalNAc- } \beta-\mathbf{1}, \text { ?-GalNAc- } \beta \text {-O-Ph (IV) }\end{array}$ & 4.5 & 37 & 1,5 & $12(\mathrm{I}+\mathrm{II}), 42(\mathrm{III}), 7.9(\mathrm{IV})^{2}$ & [48] \\
\hline $\begin{array}{l}\text { AoHex (A. oryzae CCF } \\
1066)\end{array}$ & $\begin{array}{l}\text { Mixture of GlcNAc and } \\
(\text { GlcNAc })_{2}-(\text { GlcNAc })_{7}\end{array}$ & $\begin{array}{l}\text { Mixture enriched in }(\mathrm{GlcNAc})_{2},(\mathrm{GlcNAc})_{6},(\mathrm{GlcNAc})_{7} \\
\text { and }(\mathrm{GlcNAc})_{8}(\text { all } \beta-\mathbf{1}, \mathbf{4})\end{array}$ & 4.5 & 37 & 9 & n.a. & {$[58]$} \\
\hline $\begin{array}{l}\text { Hex99 (Alteromonas sp. } \\
\left.\text { O-7 }{ }^{20}\right)\end{array}$ & $(\mathrm{GlcNAc})_{2}(24)$ & GlcNAc- $\beta-\mathbf{1}, \mathbf{6}-$ GlcNAc & 7.5 & 50 & 0.5 & n.a. & [59] \\
\hline $\begin{array}{l}\mathrm{NoHex}(\mathrm{N} \text {. orientalis } \\
\left.\quad \mathrm{IFO} 12806^{8}\right)\end{array}$ & $(\mathrm{GlcNAc})_{2}(590)$ & GlcNAc- $\beta-\mathbf{1}, 6-G l c N A c(\mathrm{I}),(\operatorname{GlcNAc})_{3}(\boldsymbol{\beta}-\mathbf{1}, \mathbf{4})(\mathrm{II})$ & 5.0 & 30 & 40,6 & 25 (I), 13 (II) ${ }^{9}$ & {$[60]$} \\
\hline $\begin{array}{l}\text { PoHex (P. oxalicum CCF } \\
\text { 2315) }\end{array}$ & pNP-GlcNPr $(46)^{17}$ & GlcNPr- $\beta-1,4-G l c N P r-\beta-O-p N P$ & 5.0 & 37 & 16 & $24^{2}$ & {$[61]$} \\
\hline $\begin{array}{l}\text { SmHex (S. marcescens } \\
\text { YS-1) }\end{array}$ & $(\mathrm{GlcNAc})_{2}(94)^{18}$ & $(\mathrm{GlcNAc})_{3}(\beta-\mathbf{1}, \mathbf{4})$ & 6.0 & 40 & 24 & $10.7^{9,10}, 14.3^{9,11}, 26.7^{9,12}$ & {$[62]$} \\
\hline \multirow[t]{7}{*}{$\begin{array}{l}\text { Tf Hex (Talaromyces flavus } \\
\text { CCF 2686) }\end{array}$} & pNP-GlcANAc (281) & GlcANAc- $\beta-1,4-$-GlcANAc- $\beta-O-p N P$ & 5.0 & 35 & 3.5 & $16^{2}$ & [63] \\
\hline & $\begin{array}{l}\text { 6-SO } \mathrm{SO}_{3}-\mathrm{GlcANAc}-\beta-\mathrm{O}-\mathrm{pNP} \\
(100)\end{array}$ & 6-SO ${ }_{3}$-GlcANAc- $\beta-1,4-6-\mathrm{SO}_{3}$-GlcANAc- $\beta-O-\mathrm{pNP}$ & 5.0 & 35 & 4 & $28^{2}$ & [63] \\
\hline & pNP-GlcNAc (50) & $\begin{array}{c}(\mathrm{GlcNAc})_{2},(\mathrm{GlcNAc})_{2}-\beta-\mathrm{O}-\mathrm{pNP},(\mathrm{GlcNAc})_{3}-\beta-\mathrm{O}-\mathrm{pNP} \\
(\text { all } \beta-\mathbf{1 , 4})\end{array}$ & 5.0 & 35 & 6 & $12^{2,13}$ & {$[64]$} \\
\hline & $\begin{array}{l}\text { 4-deoxy-GlcNAc- } \beta-O-P h \\
\text { (200) }\end{array}$ & 4-deoxy-GlcNAc- $\beta-1,6-4-$ deoxy-GlcNAc-O-Ph & 5.0 & 35 & 2 & $14^{2}$ & {$[65]$} \\
\hline & pNP-GlcNFo (50) ${ }^{17}$ & GlcNFo- $\beta-1,4-G l c N F o-\beta-O-p N P$ & 5.0 & 37 & 3 & $16^{2}$ & {$[61]$} \\
\hline & pNP-GlcNGl (50) ${ }^{19}$ & GlcNGl- $\beta$-1,4-GlcNGl- $\beta$-O-pNP & 5.0 & 37 & 1.7 & $78^{2}$ & [61] \\
\hline & GlcNAc- $\beta-N_{3}(600)$ & $\begin{array}{l}\text { GlcNAc- } \beta-\mathbf{1}, \mathbf{4}-\text { GlcNAc- } \beta-\mathrm{N}_{3} \text { (I), } \\
\text { GlcNAc- } \beta-\mathbf{1}, \mathbf{6}-\text { GlcNAc- } \beta-\mathrm{N}_{3} \text { (II) }\end{array}$ & 5.0 & 35 & 7.5 & 32 (I), 16 (II) ${ }^{2}$ & [66] \\
\hline a) $\mathrm{Y} 470 \mathrm{~F}$ & pNP-GlcNAc (50) & $\begin{array}{c}(\mathrm{GlcNAc})_{2}-\beta-O-\mathrm{pNP},(\mathrm{GlcNAc})_{3}-\beta-O-\mathrm{pNP} \\
(\mathrm{GlcNAc})_{4}-\beta-O-\mathrm{pNP}(\text { all } \beta-\mathbf{1}, \mathbf{4})\end{array}$ & 5.0 & 35 & 6 & $41^{2,14}$ & {$[64]$} \\
\hline b) $\mathrm{Y} 470 \mathrm{H}$ & pNP-GlcNAc (50) & $\begin{array}{l}(\mathrm{GlcNAc})_{2}-\beta-O-\mathrm{pNP},(\mathrm{GlcNAc})_{3}-\beta-\mathrm{O}-\mathrm{pNP} \\
(\mathrm{GlcNAc})_{4}-\beta-O-\mathrm{pNP}(\text { all } \beta-\mathbf{1}, \mathbf{4})\end{array}$ & 5.0 & 35 & 6 & $26^{2,14}$ & [64] \\
\hline c) $\mathrm{Y} 470 \mathrm{~N}$ & pNP-GlcNAc (50) & $(\text { GlcNAc) })_{7}-\beta-O-p N P$ and longer $($ all $\beta-\mathbf{1}, \mathbf{4})$ & 5.0 & 35 & 6 & n.a. ${ }^{14}$ & {$[64]$} \\
\hline VsHex (Vibrio sp. P-6-1) & $\begin{array}{l}(\mathrm{GlcNAc})_{2},(\mathrm{GlcNAc})_{3} \\
(\mathrm{GlcNAc})_{4}(2 \text { each })\end{array}$ & Unidentified oligosaccharides (all $\boldsymbol{\beta}-\mathbf{1}$, ?) ${ }^{15}$ & 6.5 & 40 & 0.5 & n.a. ${ }^{15}$ & [67] \\
\hline
\end{tabular}

${ }^{1}$ donor based; ${ }^{2}$ isolated yield(s); ${ }^{3}$ purified from $\beta$-galactosidase preparation (grade XI, Sigma-Aldrich); ${ }^{4}$ isolated from reaction with MeCN; ${ }^{5}$ from $(\mathrm{GlcNAc})_{4} ;{ }^{6}$ from $(\mathrm{GlcNAc})_{3}$; ${ }^{7}$ purified from Taka-diastase (Sankyo); ${ }^{8}$ or Amycolatopsis orientalis IFO12806T; ${ }^{9}$ product(s) not isolated; ${ }^{10}{ }^{\text {w/o }}$ co-solvent; ${ }^{11}$ co-solvent: $1,2,4$-butanetriol; ${ }^{12}$ co-solvent: 1,3 -butanediol;

${ }_{13}$ overall yield; ${ }^{14}$ no product hydrolysis; ${ }^{15}$ only detected by TLC; ${ }^{16} 491 \mathrm{mM}\left(\mathrm{NH}_{4}\right)_{2} \mathrm{SO}_{4}$, MeCN or dioxane (20\% (v/v) each) added; ${ }^{17} 45 \%(v / v)$ MeCN added; ${ }^{18} 8 \%(v / v) 1,3$-butanediol or 1,2,4-butanetriol added; ${ }^{19}$ \% $(v / v)$ MeCN added; ${ }^{20}$ or Pseudoalteromonas piscicida. 
<smiles>CC(=O)NCC(COC(O)C(O)O)Oc1ccc([N+](=O)[O-])cc1</smiles>

pNP-GIcNAc<smiles>CC(=O)NC1COC(O)C(O)C(O)C1N</smiles>

GICNAC- $\mathrm{N}_{3}$<smiles>CC1=NC2OC(O)C(O)C(O)C1O2</smiles><smiles>CC(=O)NC1COC(O)C(O)C(O)C1Oc1ccccc1</smiles>

GlcNAc-Ph<smiles>CC(=O)NC(NC(C)=O)C(O)COC(O)C1COC(O)C(O)C(O)C1O</smiles>

$(\mathrm{GlcNAc})_{2}$<smiles>CC(=O)NC1COC(Oc2ncccc2[N+](=O)[O-])C2COC1C(O)O2</smiles>

GlcNAc-NPy<smiles>CC(=O)NC1COC(Oc2ccccc2[N+](=O)[O-])C2COC(O)C(O)C1O2</smiles>

oNP-GIcNAc

Figure 3. GlcNAc donor substrates used in GH20-catalyzed trans-glycosylation reactions.

\subsection{1. $p$-Nitrophenyl Activated Donors}

The $p$-nitrophenyl (pNP) derivates of hexosaminides (Figure 3: pNP-GlcNAc) are by far the most popular donor molecules for trans-glycosylation reactions (Tables 3-6). The pNP-glycosides were developed as colorimetric substrates to study hydrolytic GH reactions, because the released pNP can be detected photometrically at $\lambda=405 \mathrm{~nm}$. However, due to their low price $(47,250 € / \mathrm{kg}$ $(16,000 € / \mathrm{mol})$ for pNP-GlcNAc at Carbosynth) [68] and not least the fact that pNP is a good leaving group, they have become attractive as donor molecules for trans-glycosylation reactions. Furthermore, the pNP-hexosaminides are accepted by a wide range of GH20 enzymes as substrate or donor molecule, including fungal (Tables 3 and 4), bacterial (Table 5), and enzymes of other origin (Table 6). Indeed, the first attempt at synthesizing a GlcNAc-containing HMO (lacto- $N$-tetraose (LNT)) utilized a pNP-activated lacto-N-biose as donor (Table 5) [69]. However, despite their popularity, the pNP-hexosaminides are not suitable as substrates for synthesis of food products (e.g., HMOs) or in other highly regulated fields, due to the toxicity of the released pNP [70].

\subsubsection{Other Activated Donors}

In addition to the phenyl-activated donors [48,65,71], other synthetic hexosaminide donor derivatives (Tables 2 and 7) have been used. These have mainly been employed to increase solubility of the donor (e.g., the 2-hydroxy-3-nitro-pyridyl activated GlcNAc (Figure 3: GlcNAc-NPy; Table 7)) [72] or to increase yield and regioselectivity (e.g., the o-nitrophenyl (oNP) derivate of $\alpha$-GlcNAc (Figure 3: oNP-GlcNAc; Table 7)) [73]. Moreover, the glycosyl azide of GlcNAc (Figure 3: GlcNAc-N ${ }_{3}$ ) has been demonstrated to be a superior donor giving higher yields compared to the conventional pNP-GlcNAc, due to its higher solubility (Tables 2 and 7) [66]. However, since the released byproducts from these donor molecules may be irritant (e.g., 2-hydroxy-3-nitro-pyridine) [74], pose an environmental hazard (e.g., oNP) [75], or be acutely toxic (e.g., phenol from Ph-activated [76] and azide from $\mathrm{N}_{3}$-activated donors [77]), none of these activated donor molecules are feasible for use in synthesis of food components or in similarly regulated applications. 
Table 3. Trans-glycosylation reactions catalyzed by GH20 $\beta$-N-acetylhexosaminidases from Aspergillus oryzae using pNP-activated carbohydrate donor substrates. New bonds are highlighted in bold.

\begin{tabular}{|c|c|c|c|c|c|c|c|c|c|}
\hline $\begin{array}{c}\text { Enzyme } \\
\text { (Organism) }\end{array}$ & $\begin{array}{l}\text { Acceptor } \\
(c[\mathrm{mM}])\end{array}$ & $\begin{array}{l}\text { Donor } \\
(c[\mathrm{mM}])\end{array}$ & $\begin{array}{l}\text { A:D } \\
\text { Ratio }\end{array}$ & Product(s) & $\mathrm{pH}$ & $T\left[{ }^{\circ} \mathrm{C}\right]$ & $t[\mathrm{~h}]$ & $\begin{array}{l}\text { Yield } \\
{[\%]^{1}}\end{array}$ & Ref \\
\hline $\begin{array}{l}\text { AoHex }(A . \\
\text { oryzae })\end{array}$ & $\begin{array}{c}\text { 6-benzyl-Gal- } \beta \text {-S-Et (118, } \\
73)\end{array}$ & $\begin{array}{l}\text { pNP-GlcNAc (96), } \\
\text { pNP-GalNAc (67) }\end{array}$ & $1: 1$ & $\begin{array}{l}\text { GlcNAc- } \beta-1,3-6-\text { benzyl-Gal- } \beta \text {-S-Et (I), } \\
\text { GalNAc- } \beta-1,3-6-\text { benzyl-Gal- } \beta \text {-S-Et (II) }\end{array}$ & 7.0 & 25 & $2.5,30$ & 2.8 (I), 5.7 (II) ${ }^{2}$ & [53] \\
\hline 3 & $\begin{array}{c}\text { Glc- } \alpha-O-p N P(41) \\
\text { GlcNAc- } \alpha-O-p N P(124)\end{array}$ & $\begin{array}{c}\text { 6-SO } \mathrm{SO}_{3}-\mathrm{GllNAc} \beta-\mathrm{O}-\mathrm{pNP} \\
(10 / 25)\end{array}$ & $\begin{array}{l}4: 1 \\
5: 1\end{array}$ & $\begin{array}{c}\text { 6-SO }{ }_{3} \text {-GlcNAc- } \beta-1,4-G l c-\alpha-O-p N P(I), \\
\text { 6-SO }{ }_{3} \text {-GlcNAc- } \beta-1,4-G l c N A c-\alpha-O-p N P \text { (II) }\end{array}$ & 6.0 & 35 & $\begin{array}{l}148, \\
168\end{array}$ & 35 (I), 94 (II) 2,4 & {$[54]$} \\
\hline 3 & $\begin{array}{l}\text { 6-SO } \mathrm{SO}_{3}-\mathrm{GlcNAc}-\beta-\mathrm{O}-\mathrm{pNP} \\
(190)\end{array}$ & pNP-GlcNAc (30) & $6: 1$ & GlcNAc- $\beta-1,4-6-\mathrm{SO}_{3}-\mathrm{GlcNAc}-\beta-O-\mathrm{pNP}$ & 6.0 & 35 & 7 & $13.4^{2}$ & {$[54]$} \\
\hline 3 & GlcNAc (1157/1112) & pNP-GlcNAc (117/111) & $10: 1$ & $\begin{array}{l}\text { GlcNAc- } \beta-\mathbf{1}, \mathbf{4}-G l c N A c \text { (I), } \\
\text { GlcNAc- } \boldsymbol{\beta}-\mathbf{1}, \mathbf{6}-\text {-GlcNAc (II) }\end{array}$ & 6.5 & 30 & 40,215 & $55(\mathrm{I})^{5}, 22(\mathrm{II})^{2}$ & [55] \\
\hline 3 & $\begin{array}{l}\text { GlcNAc- } \alpha-O-M e(531) \\
\text { GlcNAc- } \beta-O-M e(567)\end{array}$ & pNP-GlcNAc (104/97) & $\begin{array}{l}5: 1 \\
6: 1\end{array}$ & $\begin{array}{l}\text { GlcNAc- } \beta-1,4-G l c N A c-\alpha-O-M e(I), \\
\text { GlcNAc- } \beta-1,4-G l c N A c-\beta-O-M e(I I)\end{array}$ & 6.5 & 30 & 120,48 & 51 (I), 24 (II) ${ }^{2}$ & {$[55]$} \\
\hline 3 & $\begin{array}{l}\alpha-\text { Glc }^{6}, \text { Glc- } \alpha-O-R ; \\
\alpha \text {-GlcNAc, GlcNAc- } \alpha-O-R \\
\quad(\text { each ca. } 1300)^{7}\end{array}$ & $\begin{array}{c}\text { 6-SO } \mathrm{SO}_{3}-\mathrm{GlcNAc}-\beta-\mathrm{O}-\mathrm{pNP} \\
(250)\end{array}$ & $5: 1$ & $\begin{array}{c}\text { 6-SO } \mathrm{SO}_{3} \text {-GlcNAc- } \beta-\mathbf{1}, 4-\mathrm{Glc}-\alpha-\mathrm{O}-\mathrm{R}\left(\mathrm{I}=\mathrm{R}^{1}, \mathrm{II}\right. \\
\left.=\mathrm{R}^{3}, \mathrm{III}=\mathrm{R}^{6}\right) \\
6-\mathrm{SO}_{3} \text {-GlcNAc- } \beta-1,4-\mathrm{GlcNAc}-\alpha-\mathrm{R}\left(\mathrm{IV}=\mathrm{R}^{5}\right. \\
\left.\mathrm{V}=\mathrm{R}^{1}, \mathrm{VI}=\mathrm{R}^{2}, \mathrm{VII}=\mathrm{R}^{3}, \mathrm{VIII}=\mathrm{R}^{4}\right)^{7}\end{array}$ & 6.0 & 35 & $120-144$ & $\begin{array}{c}17 \text { (I), } 34 \text { (II), } 36 \text { (III), } \\
38 \text { (IV), } 51 \text { (V), } 87 \text { (VI), } \\
92 \text { (VII), } 93 \text { (VIII) }{ }^{2}\end{array}$ & {$[78]$} \\
\hline 3 & $\begin{array}{l}\text { Glc- } \alpha-O-R^{3}, \text { GlcNAc- } \alpha- \\
O-R^{3}, \text { GlcNAc, } \\
\text { Glc- } \alpha-O-R^{1}, \text { Gal- } \alpha-O-R^{3} \\
(\text { each ca. } 1700)^{7}\end{array}$ & $\begin{array}{c}\text { 6-SO } \mathrm{SO}_{3}-\mathrm{GlcNAc}-\beta-\mathrm{O}-\mathrm{pNP} \\
(178)\end{array}$ & $10: 1$ & 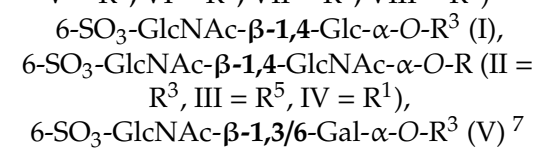 & 6.0 & 35 & $\begin{array}{l}74,66 \\
370,71 \\
48\end{array}$ & $\begin{array}{l}38 \text { (I), } 92 \text { (II), } 38 \text { (III) } \\
17 \text { (IV), } 25(\mathrm{~V})^{2}\end{array}$ & [79] \\
\hline & GlcNAc- $\beta-N-A c(206)$ & pNP-GlcNAc (21) & $10: 1$ & $\begin{array}{l}\text { GlcNAc- } \beta-1,4-G l c N A c-\beta-N-A c \\
\text { GalNAc- } \beta-1,3 / 4-G l c-\beta-O-M e(I=\beta-1,3 \\
\text {, II }\end{array}$ & 5.0 & 37 & 4 & $17^{2,12}$ & [49] \\
\hline 3 & $\begin{array}{c}\text { Glc- } \beta \text {-O-Me, Glc- } \alpha-O-M e \\
\text { (603 each) }\end{array}$ & $\begin{array}{l}\text { pNP-GalNAc, } \\
\text { pNP-GlcNAc (60) }\end{array}$ & $10: 1$ & $\begin{array}{c}=\beta-1,4), \text { GalNAc- } \beta-1,4 / 6-G l c-\alpha-O-M e(I I I \\
=\beta-1,4, \text { IV }=\beta-1,6) \\
\text { GlcNAc- } \beta-1,3 / 4-G l c-\beta-O-M e(V=\beta-1,3, \text { VI } \\
=\beta-1,4), \text { GlcNAc- } \beta-1,4 / 6-G l c-\alpha-O-M e(V I I \\
=\beta-1,4, \text { VII }=\beta-1,6)\end{array}$ & 6.5 & 28 & 24 & $\begin{array}{c}49(\mathrm{I}+\mathrm{II}), 36 \text { (II I+ IV) } \\
23(\mathrm{~V}+\mathrm{VI}), 17 \text { (VII + } \\
\text { VIII) }{ }^{2}\end{array}$ & {$[80]$} \\
\hline 3 & $\begin{array}{c}\text { GlcNAc- } \beta-O-M e \\
\text { GlcNAc- } \alpha-O-M e(568 \\
\text { each) }\end{array}$ & pNP-GalNAc (104) & $6: 1$ & $\begin{array}{c}\text { GalNAc- } \beta-\mathbf{1}, \mathbf{4} / 6-\text { GlcNAc- } \alpha \text {-O-Me }(\mathrm{I}= \\
\beta-\mathbf{1}, \mathbf{4}, \mathrm{II}=\beta-\mathbf{1}, \mathbf{6}) \\
\text { GalNAc- } \beta-\mathbf{1}, \mathbf{4}-\mathrm{GlcNAc}-\beta-\mathrm{O}-\mathrm{Me}(\mathrm{III}), \text { one } \\
\text { unidentified isomer }\end{array}$ & 4.5 & 30 & 96 & $89(\mathrm{I}+\mathrm{II})^{2}$ & {$[81]$} \\
\hline 3 & $\begin{array}{c}\text { GlcNAc, GlcNAc- } \beta-O-M e \\
\text { GlcNAc- } \alpha-O-M e(772 \\
\text { each) }\end{array}$ & pNP-GlcNAc (76) & $10: 1$ & $\begin{array}{c}\text { GlcNAc- } \beta-\mathbf{1}, \mathbf{4} / \mathbf{6}-\text {-GlcNAc }(\mathrm{I}=\boldsymbol{\beta}-\mathbf{1}, \mathbf{4}, \mathrm{II}= \\
\beta-\mathbf{1} \mathbf{6}), \text { GlcNAc- } \beta-\mathbf{1}, \mathbf{4}-\mathrm{GlcNAc}-\alpha-\mathrm{O}-\mathrm{Me}(\mathrm{III}), \\
\text { GlcNAc- } \beta-\mathbf{1}, \mathbf{4}-\mathrm{GlcNAc}-\beta-O-M e(\mathrm{IV}), \text { one } \\
\text { unidentified isomer }\end{array}$ & 6.5 & 30 & n.a. & $\begin{array}{l}55 \text { (I) }{ }^{8}, 22 \text { (II) }{ }^{8}, 55 \\
\text { (III), } 24 \text { (IV) }{ }^{2}\end{array}$ & {$[82]$} \\
\hline 3 & Man (3996) & pNP-GlcNAc $(38+29)^{9}$ & $105: 1$ & $\begin{array}{c}\text { GlcNAc- } \beta-1,1 / 3 / 4 / 6-\operatorname{Man}(\mathrm{I}=\boldsymbol{\beta - 1 , 1}, \mathrm{II}= \\
\beta-1,3, \mathrm{III}=\boldsymbol{\beta}-1,4, \mathrm{IV}=\boldsymbol{\beta}-\mathbf{1}, \mathbf{6})\end{array}$ & 5.0 & 37 & 4 & $24.6(\mathrm{I}+\mathrm{II}+\mathrm{III}+\mathrm{IV})^{2}$ & [83] \\
\hline
\end{tabular}


Table 3. Cont

\begin{tabular}{|c|c|c|c|c|c|c|c|c|c|}
\hline $\begin{array}{c}\text { Enzyme } \\
\text { (Organism) }\end{array}$ & $\begin{array}{l}\text { Acceptor } \\
(c[\mathrm{mM}])\end{array}$ & $\begin{array}{l}\text { Donor } \\
(c[\mathrm{mM}])\end{array}$ & $\begin{array}{l}\text { A:D } \\
\text { Ratio }\end{array}$ & Product(s) & $\mathrm{pH}$ & $T\left[{ }^{\circ} \mathrm{C}\right]$ & $t[\mathrm{~h}]$ & $\begin{array}{l}\text { Yield } \\
{[\%]^{1}}\end{array}$ & Ref. \\
\hline 3 & GalNAc (988/983) & $\begin{array}{l}\text { pNP-GlcNAc (140), } \\
\text { pNP-GalNAc (141) }\end{array}$ & $7: 1$ & $\begin{array}{l}\text { GlcNAc- } \boldsymbol{\beta}-\mathbf{1}, \mathbf{6}-\text { GalNAc (I), } \\
\text { GlcNAc- } \boldsymbol{\beta}-\mathbf{1} \mathbf{4}-\text {-GlcNAc (II), } \\
\text { GalNAc- } \boldsymbol{\beta}-\mathbf{1}, \mathbf{6}-\text { GalNAc (III) }\end{array}$ & $\begin{array}{l}6.5 \\
4.5\end{array}$ & 30 & 30,47 & $26(\mathrm{I})^{5}, 38(\mathrm{III})^{2}$ & [84] \\
\hline \multirow[t]{7}{*}{$\begin{array}{l}\text { AoHex }(A \text {. } \\
\text { oryzae CCF } \\
1066)\end{array}$} & $\begin{array}{c}\text { GlcNAc- } \beta \text {-linker- } \beta \text {-GlcNAc } \\
(315,118)\end{array}$ & $\begin{array}{l}\text { pNP-GlcNAc (146), } \\
\text { pNP-GalNAc (292) }\end{array}$ & $\begin{array}{l}2: 1 \\
1: 2\end{array}$ & $\begin{array}{l}\text { GlcNAc- } \beta-\mathbf{1}, 4-G l c N A c-\beta \text {-linker- } \beta \text {-GlcNAc } \\
\text { (I), } \\
\text { GalNAc- } \beta-1,4-G l c N A c-\beta \text {-linker- } \beta \text {-GlcNAc } \\
\text { (II) }\end{array}$ & 5.0 & 35 & 4.5 & 7 (I), 6 (II) $)^{2}$ & [85] \\
\hline & GlcNAc (230) & pNP-GalNAc (60) & $4: 1$ & GalNAc- $\beta-1,4-G l c N A c$ & 5.0 & 37 & 1 & $84.5^{2}$ & [86] \\
\hline & $\begin{array}{l}\text { GlcNAc- } \alpha-O-R^{3}(115) \\
\text { Gal- } \beta-1,4-\text { GlcNAc- } \alpha- \\
\quad O-R^{3}(83)^{7}\end{array}$ & pNP-GalNAc (29) & $\begin{array}{l}4: 1 \\
3: 1\end{array}$ & $\begin{array}{l}\text { GalNAc- } \beta-1,4-G l c N A c-\alpha-O-R^{3}(\mathrm{I}), \\
\text { GalNAc- } \beta-\mathbf{6} \text {-GalNAc- } \beta-1,4-G \text {-GlcNAc- } \alpha-O-R^{3} \\
\text { (II) }{ }^{7}\end{array}$ & 5.0 & 37 & $4,1.5$ & 78 (I), 17 (II) ${ }^{2}$ & [87] \\
\hline & $\begin{array}{c}\text { GlcNAc- } \beta-1,4-\mathrm{ManNAc} \\
(96)\end{array}$ & pNP-GalNAc (67) & $1: 1$ & GalNAc- $\beta-\mathbf{1}, 4-G l c N A c-\beta-1,4-M a n N A c$ & 5.0 & 37 & 2 & $41^{2,10}$ & [88] \\
\hline & $\begin{array}{l}\text { Gal (223), GalNAc (197), } \\
\text { Lac (189) }\end{array}$ & pNP-GlcNAc (28/38/24) & $\begin{array}{l}8: 1 \\
5: 1\end{array}$ & $\begin{array}{c}\text { GlcNAc- } \beta-\mathbf{1}, \mathbf{1}-G a l \text { (I), } \\
\text { GlcNAc- } \beta-\mathbf{1}, \mathbf{4}-\text { GlcNAc- } \beta-\mathbf{1}, \mathbf{1}-\text { Gal (II), } \\
\text { GlcNAc- } \beta-\mathbf{1}, \mathbf{6}-\text {-GalNAc (III), } \\
\text { Lac- } \alpha / \beta-1,1-G l c N A c\end{array}$ & 5.0 & 37 & $4.5,5$ & 17 (I), 7 (II), 14 (III) ${ }^{2}$ & [89] \\
\hline & $\begin{array}{l}\text { GlcNAc- } \beta-1,4-M a n N A c \\
(104 / 96)\end{array}$ & $\begin{array}{l}\text { pNP-GlcNAc (65), } \\
\text { pNP-GalNAc (67) }\end{array}$ & $\begin{array}{l}2: 1 \\
1: 1\end{array}$ & $\begin{array}{l}\text { GlcNAc- } \beta-\mathbf{1}, 4-G l c N A c-\beta-1,4-M a n N A c \text { (I), } \\
\text { GalNAc- } \beta-\mathbf{1 , 4}-\text {-GlcNAc- } \beta-1,4-M a n N A c \text { (II) }\end{array}$ & 5.0 & 37 & $0.8,2$ & 36 (I), 41 (II) $)^{2,10}$ & [90] \\
\hline & pNP-GlcNAc (24) & pNP-GlcNPr (46) & $1: 2$ & $\begin{array}{l}\text { GlcNPr- } \beta-\mathbf{1}, 4-G l c N P r-\beta-O-p N P(I), \\
\text { GlcNPr- } \beta-\mathbf{1}, 4-G l c N A c-\beta-O-p N P \text { (II) }\end{array}$ & 5.0 & 37 & 15.5 & 4.3 (I), 1.8 (II) $)^{2,11}$ & [61] \\
\hline
\end{tabular}

${ }^{1}$ donor based; ${ }^{2}$ isolated yield(s); ${ }^{3}$ purified from $\beta$-galactosidase preparation (grade XI, Sigma-Aldrich); ${ }^{4} 10 \%(v / v)$ MeCN added $;{ }^{5}$ undesired regioisomer digested; ${ }^{6}$ no product formation; ${ }^{7} \mathrm{R}^{1}=\mathrm{Me}, \mathrm{R}^{2}=\mathrm{Pr}, \mathrm{R}^{3}=$ allyl, $\mathrm{R}^{4}=\mathrm{pNP}, \mathrm{R}^{5}=\mathrm{OH}, \mathrm{R}^{6}=\mathrm{Ph}^{8}{ }^{8}$ first product I formed, later product II; ${ }^{9}$ donor added stepwise; ${ }^{10} 9 \%(v / v) \mathrm{MeCN}$ added; ${ }^{11} 45 \%(v / v) \mathrm{MeCN}$ added; 12 isolated as peracetylated derivate(s) 
Table 4. Trans-glycosylation reactions catalyzed by fungal GH20 $\beta$-N-acetyl hexosaminidases using pNP-activated carbohydrate donor substrates. New bonds are highlighted in bold.

\begin{tabular}{|c|c|c|c|c|c|c|c|c|c|}
\hline $\begin{array}{c}\text { Enzyme } \\
\text { (Organism) }\end{array}$ & $\begin{array}{l}\text { Acceptor } \\
(c[\mathrm{mM}])\end{array}$ & $\begin{array}{c}\text { Donor } \\
(c[\mathrm{mM}])\end{array}$ & $\begin{array}{l}\text { A:D } \\
\text { Ratio }\end{array}$ & Product(s) & $\mathrm{pH}$ & $T\left[{ }^{\circ} \mathrm{C}\right]$ & $t[\mathrm{~h}]$ & $\begin{array}{l}\text { Yield } \\
{[\%]^{1}}\end{array}$ & Ref. \\
\hline $\begin{array}{l}\text { AfHex } \\
\text { (Aspergillus } \\
\text { flavofurcatis } \\
\text { CCF 3061) }\end{array}$ & $\begin{array}{l}\text { Gal (223), GalNAc (197), } \\
\text { Lac (189) }\end{array}$ & pNP-GlcNAc (28/38/24) & $\begin{array}{l}8: 1 \\
5: 1\end{array}$ & 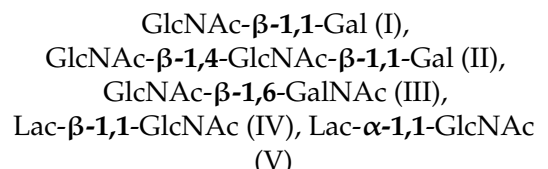 & 5.0 & 37 & $\begin{array}{l}4.5,5 \\
7\end{array}$ & $\begin{array}{l}22 \text { (I) }{ }^{2}, 23.5(\mathrm{II})^{2}, 20 \\
\text { (III) }^{2}, 10(\mathrm{IV})^{2,3} \\
9(\mathrm{~V})^{2,3}\end{array}$ & [89] \\
\hline $\begin{array}{c}\text { AtHex } \\
\text { (Aspergillus } \\
\text { tamarii CCF } \\
\text { 1665) }\end{array}$ & $\begin{array}{l}\text { Gal (223), GalNAc (197), } \\
\text { Lac (189) }\end{array}$ & pNP-GlcNAc (28/38/24) & $\begin{array}{l}8: 1 \\
5: 1\end{array}$ & 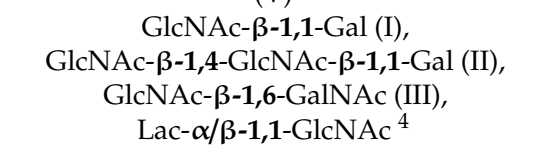 & 5.0 & 37 & $4.5,5$ & 12 (I), 6 (II), 17.5 (III) ${ }^{2}$ & [89] \\
\hline $\begin{array}{l}\text { PbHex } \\
\text { (Penicillium } \\
\text { brasilianum } \\
\text { CCF 2155) }\end{array}$ & $\begin{array}{l}\text { 6-Ac-GlcNAc (380), } \\
\text { GlcNAc (452) }\end{array}$ & $\begin{array}{c}\text { pNP-GlcNAc (42), } \\
\text { 6-Ac-GlcNAc- } \beta-O-p N P \\
(40)\end{array}$ & $\begin{array}{r}9: 1 \\
11: 1\end{array}$ & $\begin{array}{l}\text { GlcNAc- } \beta-1,4-6-O-A c-G l c N A c \text { (I), } \\
\text { 6-O-Ac-GlcNAc- } \beta-1,4-G l c N A c \text { (II) }\end{array}$ & 5.5 & 37 & $2,4.5$ & 16.5 (I), 21 (II) ${ }^{2,5}$ & [91] \\
\hline $\begin{array}{l}\text { PoHex }(P . \\
\text { oxalicum })\end{array}$ & GlcNAc (300) & pNP-GlcNAc (30) & $10: 1$ & GlcNAc- $\beta-\mathbf{1}, \mathbf{4}-$ GlcNAc & 5.0 & 35 & 5.25 & $13^{2}$ & [92] \\
\hline & GalNAc (500) & pNP-GalNAc $(30+22)^{6}$ & $17: 1$ & GalNAc- $\beta-1,6-G a l N A c$ & 5.0 & 35 & 6.25 & $34^{2}$ & [92] \\
\hline $\begin{array}{l}\text { PoHex }(P . \\
\text { oxalicum } \\
\text { CCF 2430) }\end{array}$ & $\begin{array}{c}\text { GlcNAc (877), GalNAc } \\
\text { (877) }\end{array}$ & pNP-GalNAc (175/292) & $\begin{array}{l}5: 1 \\
3: 1\end{array}$ & $\begin{array}{l}\text { GalNAc- } \boldsymbol{\beta - 1} \mathbf{1 , 4 - G l c N A c} \text { (I), } \\
\text { GalNAc- } \boldsymbol{\beta}-\mathbf{1} \mathbf{6} \text {-GlcNAc (II), } \\
\text { GalNAc- } \boldsymbol{\beta} \mathbf{- 1 , 6 - G a l N A c ~ ( I I I ) ~}\end{array}$ & 4.5 & 37 & 3.5 & 26.5 (I), 19 (II), 87 (III) & {$[87]$} \\
\hline \multirow[t]{4}{*}{$\begin{array}{l}T f \operatorname{Hex}(T \text {. } \\
\text { flavus CCF } \\
2686)\end{array}$} & GlcNAc (350) & $\begin{array}{l}\text { 6-OH-GlcNAc- } \beta-O-p N P \\
(50)\end{array}$ & $7: 1$ & GlcANAc- $\beta-1,4-G l c N A c$ & 5.0 & 37 & 4 & $23^{2,8}$ & [63] \\
\hline & GlcNAc (300) & $\begin{array}{c}6-\mathrm{SO}_{3}-\mathrm{GlcNAc}-\beta-\mathrm{O}-\mathrm{pNP} \\
(75)\end{array}$ & $4: 1$ & 6-SO ${ }_{3}-\mathrm{GlcNAc}_{-} \beta-1,4-\mathrm{GlcNAc}$ & 5.0 & 35 & 6.5 & $33^{2}$ & [63] \\
\hline & GlcNAc (300) & $\begin{array}{c}\text { 6-OH-GlcNAc- } \beta-O-p N P \\
(30)\end{array}$ & $10: 1$ & GlcANAc- $\beta-1,4-G l c N A c$ & 5.0 & 37 & 4.5 & $37^{2,8}$ & [93] \\
\hline & $\begin{array}{c}\text { GlcNAc- } \beta-1,4-M a n N A c \\
(30)\end{array}$ & $\begin{array}{l}\text { 6-OH-GalNAc- } \beta-O-p N P \\
\text { (73) }\end{array}$ & $1: 2$ & $\begin{array}{l}\text { GalANAc- } \beta-\mathbf{1}, \mathbf{4}-\text { GlcNAc- } \beta-1,4-M a n N A c \\
\text { (I), GalANAc- } \beta-\mathbf{1}, \mathbf{4}-\text { GlcNAc (II) }\end{array}$ & 5.0 & 35 & 5 & $\begin{array}{l}35(\mathrm{I}), \\
39 \text { (II) }\end{array}$ & {$[88]$} \\
\hline $\begin{array}{l}T f \operatorname{Hex}(T \\
\text { flavus CCF } \\
2686)\end{array}$ & $\begin{array}{l}\text { GlcNac, GalNAc, Glc, Gal } \\
(300)\end{array}$ & $\begin{array}{c}\text { pNP-GlcNAc, } \\
\text { pNP-GalNAc (50) }\end{array}$ & $6: 1$ & $\begin{array}{c}\text { GlcNAc- } \beta-\mathbf{- 1 , 4 - G l c N A c}(\mathrm{I}), \\
\text { GalNAc- } \beta-\mathbf{1 , 6} \text {-GalNAc (II), } \\
\text { GlcNAc- } \boldsymbol{\beta}-\mathbf{1 , 1 - G l c}(\mathrm{III}), \text { GlcNAc- } \boldsymbol{\beta}-\mathbf{1 , 1 - G a l} \\
\text { (IV) }\end{array}$ & 5.0 & 35 & 5 & $\begin{array}{l}24 \text { (I), } 31 \text { (II), } 14 \text { (III) } \\
10 \text { (IV) }\end{array}$ & [94] \\
\hline a) $\mathrm{Y} 470 \mathrm{~F}$ & GlcNAc- $\beta$-linker (150) & pNP-GalNAc $(50+50)^{6}$ & $3: 1$ & GalNAc- $\beta-1,4$-GlcNAc- $\beta$-linker & 5.0 & 35 & 8.5 & $51^{2,9}$ & [95] \\
\hline
\end{tabular}


Table 4. Cont

\begin{tabular}{|c|c|c|c|c|c|c|c|c|c|}
\hline $\begin{array}{l}\text { Enzyme } \\
\text { (Organism) }\end{array}$ & $\begin{array}{l}\text { Acceptor } \\
(c[\mathrm{mM}])\end{array}$ & $\begin{array}{l}\text { Donor } \\
(c[\mathrm{mM}])\end{array}$ & $\begin{array}{l}\text { A:D } \\
\text { Ratio }\end{array}$ & Product(s) & $\mathrm{pH}$ & $T\left[{ }^{\circ} \mathrm{C}\right]$ & $t[\mathrm{~h}]$ & $\begin{array}{l}\text { Yield } \\
{[\%]^{1}}\end{array}$ & Ref. \\
\hline b) $\mathrm{Y} 470 \mathrm{H}$ & GlcNAc- $\beta-\mathrm{N}_{3}(200)$ & pNP-GalNAc (50) & $4: 1$ & $\begin{array}{l}\text { GalNAc- } \beta-1,4-G l c N A c-\beta-N_{3} \\
\text { GlcNAc- } \beta-1,4-G l c N A c-\beta-N_{3}\end{array}$ & 5.0 & 45 & 6.5 & n.a. ${ }^{4,10}$ & [96] \\
\hline \multirow{4}{*}{ c) $Y 470 \mathrm{~N}$} & $\begin{array}{c}\text { GlcNAc }(200) \\
\text { GlcNAc- } \beta-O-\mathrm{EtN}_{3}(150) \\
\text { GlcNAc- } \beta-\mathrm{N}_{3}(200)\end{array}$ & pNP-GalNAc (50) & $\begin{array}{l}4: 1 \\
3: 1\end{array}$ & $\begin{array}{c}\text { GalNAc- } \beta-\mathbf{1}, 4-G l c N A c(\mathrm{I}), \\
\text { GalNAc- } \beta-\mathbf{1}, \mathbf{4}-\mathrm{GlcNAc}-\beta-\mathrm{O}-\mathrm{EtN}_{3} \text { (II), } \\
\text { GalNAc- } \beta-\mathbf{1}, \mathbf{4}-\mathrm{GlcNAc}-\beta-\mathrm{N}_{3} \text { (III) }\end{array}$ & 5.0 & 45 & 4 & 58 (I), 48 (II), 35 (III) & [97] \\
\hline & MurNAc- $\beta-O-\operatorname{Pr}(100)$ & pNP-GalNAc (50) & 2:1 & GalNAc- $\beta-1,6-$ MurNAc- $\beta-O-P r$ & 5.0 & 35 & 5 & 1 & [94] \\
\hline & GlcNAc- $\beta$-linker (50) & pNP-GlcNAc $(50+25)^{6}$ & $1: 1$ & $\begin{array}{l}(\mathrm{GlcNAc})_{2}-\beta \text {-linker }(\mathrm{I}),(\mathrm{GlcNAc})_{3}-\beta \text {-linker } \\
(\mathrm{II}),(\mathrm{GlcNAc})_{4}-\beta \text {-linker (III) (all } \beta \text {-1,4) } \\
\left.(\mathrm{GlcNAc})_{2}-\beta \text {-linker (I), (GlcNAc) }\right)_{3}-\beta \text {-linker }\end{array}$ & 5.0 & 35 & 8 & $26.8(\mathrm{I}+\mathrm{II}+\mathrm{III})^{2}$ & [64] \\
\hline & GlcNAc- $\beta-O-\mathrm{EtN}_{3}(100)$ & pNP-GlcNAc $(50+50)^{6}$ & 2:1 & 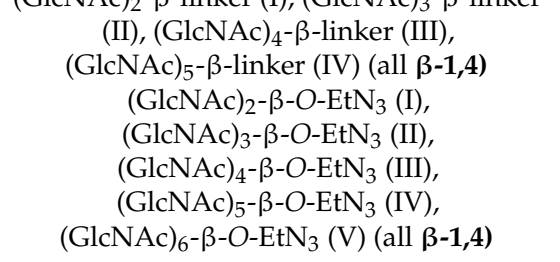 & 5.0 & 35 & 8 & $\begin{array}{l}21.5 \text { (I), } 18 \text { (II), } 11.1 \\
\text { (III), } 2.4(\mathrm{IV}), 3.4(\mathrm{~V})^{2}\end{array}$ & [98] \\
\hline $\begin{array}{c}\text { ThHex } \\
\text { (Trichoderma } \\
\text { harzianum } \\
\text { CCF 2687) }\end{array}$ & UDP-GlcNAc (32) & pNP-GalNAc (208) & 1:5 & GalNAc- $\beta-1,4-G l c N A c-\alpha$-UDP & 6.5 & 30 & 8 & $22^{2,11}$ & [100] \\
\hline
\end{tabular}


Table 5. Trans-glycosylation reactions catalyzed by bacterial GH20 enzymes using pNP-activated carbohydrate donor substrates. New bonds are highlighted in bold.

\begin{tabular}{|c|c|c|c|c|c|c|c|c|c|}
\hline Enzyme (Organism) & $\begin{array}{l}\text { Acceptor } \\
(c[\mathrm{mM}])\end{array}$ & $\begin{array}{l}\text { Donor } \\
(c[\mathrm{mM}])\end{array}$ & $\begin{array}{l}\text { A:D } \\
\text { Ratio }\end{array}$ & Product(s) & $\mathrm{pH}$ & $T\left[{ }^{\circ} \mathrm{C}\right]$ & $\begin{array}{c}t \\
{[\mathrm{~h}]}\end{array}$ & $\begin{array}{l}\text { Yield } \\
{[\%]^{1}}\end{array}$ & Ref. \\
\hline $\begin{array}{c}\text { AuLnb (Aureobacterium sp. } \\
\text { L-101) }\end{array}$ & Lac (182) & pNP-LNB (18) & $10: 1$ & LNT $(\beta-\mathbf{1}, \mathbf{3})$ & 5.5 & 40 & 5 & $3.7^{2}$ & [69] \\
\hline \multirow[t]{4}{*}{ BbhI (B. bifidum JCM 1254) } & Lac (400) & pNP-GlcNAc (20) & $20: 1$ & LNT II $(\beta-\mathbf{1}, \mathbf{3})$ & 5.8 & 55 & 1.5 & $46^{3,7}$ & {$[101]$} \\
\hline & Lac (40) & pNP-GlcNAc (10) & $4: 1$ & LNT II $(\beta-1,3)$ & 7.0 & 37 & 0.6 & $16^{3}$ & {$[102]$} \\
\hline & Lac $(400)$ & pNP-GlcNAc (20) & $20: 1$ & LNT II $(\beta-1,3)$ & 5.8 & 55 & 1.5 & $45^{2,7}$ & {$[103]$} \\
\hline & Lac $(400)$ & pNP-GalNAc (10) & $40: 1$ & GalNAc- $\beta-1,3-L a c$ & 5.8 & 45 & 4 & $55^{2,7}$ & [103] \\
\hline a) $\mathrm{R} 577 \mathrm{~K}$ & Lac (40) & pNP-GlcNAc (10) & $4: 1$ & LNT II $(\beta-1,3)$ & 7.0 & 37 & 0.6 & $36^{3}$ & [102] \\
\hline b) $\mathrm{H} 603 \mathrm{~F}$ & Lac (40) & pNP-GlcNAc (10) & $4: 1$ & LNT II $(\beta-1,3)$ & 7.0 & 37 & n.a. & n.a. ${ }^{3}$ & {$[102]$} \\
\hline c) $\mathrm{D} 606 \mathrm{~N}$ & $\operatorname{Lac}(40)$ & pNP-GlcNAc (10) & $4: 1$ & LNT II $(\beta-1,3)$ & 7.0 & 37 & n.a. & $9^{3}$ & {$[102]$} \\
\hline \multirow[t]{2}{*}{ d) $\mathrm{D} 746 \mathrm{~A}$} & Lac (400) & pNP-GlcNAc (20) & $20: 1$ & LNT II $(\beta-1,3)$ & 5.8 & 55 & 8 & $40^{3,4,7}$ & [101] \\
\hline & Lac (400) & pNP-GlcNAc (20) & $20: 1$ & LNT II $(\beta-1,3)$ & 5.8 & 37 & n.a. & $58^{3,7}$ & [104] \\
\hline e) $\mathrm{D} 746 \mathrm{C} / \mathrm{G} / \mathrm{S} / \mathrm{V}$ & Lac (400) & pNP-GlcNAc (20) & $20: 1$ & LNT II $(\beta-1,3)$ & 5.8 & 37 & n.a. & $62-73^{3,7}$ & [104] \\
\hline \multirow[t]{2}{*}{ f) D746E } & Lac (400) & pNP-GlcNAc (20) & $20: 1$ & LNT II $(\beta-1,3)$ & 5.8 & 55 & 1 & $71^{3,7}$ & [101] \\
\hline & Lac (400) & pNP-GlcNAc (20) & $20: 1$ & LNT II $(\beta-1,3)$ & 5.8 & 37 & n.a. & $63^{3,7}$ & [104] \\
\hline g) D746I & Lac (400) & pNP-GlcNAc (20) & $20: 1$ & LNT II $(\beta-1,3)$ & 5.8 & 37 & n.a. & $46^{3,7}$ & [104] \\
\hline \multirow[t]{2}{*}{ h) D746Q } & Lac (400) & pNP-GlcNAc (20) & $20: 1$ & LNT II $(\beta-1,3)$ & 5.8 & 55 & 6 & $3^{3,4,7}$ & [101] \\
\hline & Lac (400) & pNP-GlcNAc (20) & $20: 1$ & LNT II $(\beta-1,3)$ & 5.8 & 37 & n.a. & 0 & [104] \\
\hline i) $\mathrm{D} 746 \mathrm{~T}$ & Lac (400) & pNP-GlcNAc (20) & $20: 1$ & LNT II $(\beta-1,3)$ & 5.8 & 37 & 3 & $85^{3,4,7}$ & [104] \\
\hline j) $\mathrm{D} 746 \mathrm{~F} / \mathrm{H} / \mathrm{K} / \mathrm{L} / \mathrm{M} / \mathrm{N} / \mathrm{P} / \mathrm{R} / \mathrm{W} / \mathrm{Y}$ & Lac (400) & pNP-GlcNAc (20) & $20: 1$ & LNT II $(\beta-1,3)$ & 5.8 & 37 & n.a. & 0 & [104] \\
\hline k) W801H & Lac (40) & pNP-GlcNAc (10) & $4: 1$ & LNT II $(\beta-1,3)$ & 7.0 & 37 & n.a. & $17^{3}$ & [102] \\
\hline 1) $W 805 \mathrm{~A} / \mathrm{G} / \mathrm{N} / \mathrm{S} / \mathrm{T} / \mathrm{V}$ & Lac (400) & pNP-GlcNAc (20) & $20: 1$ & LNT II $(\beta-1,3)$ & 5.8 & 37 & n.a. & $45-48^{3,7}$ & [104] \\
\hline m) W805C/I/K/L/P & Lac (400) & pNP-GlcNAc (20) & $20: 1$ & LNT II $(\beta-\mathbf{1}, \mathbf{3})$ & 5.8 & 37 & n.a. & $60-69^{3,7}$ & [104] \\
\hline n) $W 805 \mathrm{D} / \mathrm{E} / \mathrm{F} / \mathrm{H} / \mathrm{M} / \mathrm{Q} / \mathrm{Y}$ & Lac (400) & pNP-GlcNAc (20) & $20: 1$ & LNT II $(\beta-1,3)$ & 5.8 & 37 & n.a. & $16-40^{3,7}$ & [104] \\
\hline o) W805R & Lac (400) & pNP-GlcNAc (20) & $20: 1$ & LNT II $(\beta-1,3)$ & 5.8 & 37 & 3 & $82^{3,7}$ & [104] \\
\hline p) $Y 827 F$ & Lac (400) & pNP-GlcNAc (20) & $20: 1$ & LNT II $(\beta-1,3)$ & 5.8 & 55 & 1 & $53^{3,7}$ & [101] \\
\hline q) $\mathrm{W} 882 \mathrm{H}$ & Lac (40) & pNP-GlcNAc (10) & $4: 1$ & LNT II $(\beta-1,3)$ & 7.0 & 37 & 1.3 & $66^{3}$ & [102] \\
\hline r) $\mathrm{D} 884 \mathrm{~N}$ & Lac (40) & pNP-GlcNAc (10) & $4: 1$ & LNT II $(\beta-1,3)$ & 7.0 & 37 & n.a. & $12^{3}$ & [102] \\
\hline s) $\mathrm{RMe}^{10}$ & $\mathrm{Lac}(400)$ & pNP-GlcNAc (20) & $20: 1$ & LNT II $(\beta-1,3)$ & 5.8 & 37 & 6 & $75^{3,7}$ & [104] \\
\hline
\end{tabular}


Table 5. Cont.

\begin{tabular}{|c|c|c|c|c|c|c|c|c|c|}
\hline Enzyme (Organism) & $\begin{array}{l}\text { Acceptor } \\
\text { (c }[\mathrm{mM}])\end{array}$ & $\begin{array}{c}\text { Donor } \\
(c[\mathrm{mM}])\end{array}$ & $\begin{array}{l}\text { A:D } \\
\text { Ratio }\end{array}$ & Product(s) & $\mathrm{pH}$ & $T\left[{ }^{\circ} \mathrm{C}\right]$ & $\begin{array}{c}t \\
{[\mathrm{~h}]}\end{array}$ & $\begin{array}{l}\text { Yield } \\
{[\%]^{1}}\end{array}$ & Ref. \\
\hline t) $\mathrm{RM} 34^{11}$ & Lac (400) & pNP-GlcNAc (20) & $20: 1$ & LNT II $(\beta-1,3)$ & 5.8 & 37 & 6 & $69^{3,7}$ & {$[104$} \\
\hline u) RMf125 12 & Lac (400) & pNP-GlcNAc (20) & $20: 1$ & LNT II $(\beta-1,3)$ & 5.8 & 37 & 6 & $81^{3,7}$ & [104 \\
\hline \multirow[t]{2}{*}{ LnbB (B. bifidum JCM 1254) } & Lac (500) & pNP-LNB (5) & $100: 1$ & $\operatorname{LNT}(\beta-1,3)$ & 4.5 & 40 & n.a. & n.d. ${ }^{3}$ & [51] \\
\hline & Lac (600) & pNP-LNB (20) & $30: 1$ & LNT $(\beta-1,3)$ & 5.8 & 37 & 0.2 & $8^{3,8}$ & {$[105$} \\
\hline a) $\mathrm{D} 320 \mathrm{E}$ & Lac (600) & pNP-LNB (20) & $30: 1$ & LNT $(\beta-1,3)$ & 5.8 & 37 & 1 & $12^{3,8}$ & {$[105$} \\
\hline b) $\mathrm{D} 320 \mathrm{~A}$ & Lac (600) & pNP-LNB (20) & $30: 1$ & LNT $(\beta-1,3)$ & 5.8 & 37 & 27 & $10^{3,8}$ & {$[105$} \\
\hline c) $\mathrm{Y} 419 \mathrm{~F}$ & Lac (600) & pNP-LNB (20) & $30: 1$ & LNT $(\beta-1,3)$ & 5.8 & 37 & 9 & $13^{3,8}$ & {$[105$} \\
\hline SpHexE314A (S. plicatus) & $\begin{array}{c}\text { pNP-3/4/6-S-GlcNAc, } \\
\text { pNP-3/4-S-GalNAc, } \\
\text { pNP-4-S-ManNAc, } \\
\text { pNP-4-S-Man (5 each) }\end{array}$ & $\begin{array}{c}\text { pNP-GlcNAc, } \\
\text { pNP-GalNAc }(30+30)^{5}\end{array}$ & $1: 6$ & Different thioglycosides $(\beta-S)^{6}$ & 7 & 37 & $<1$ & $>999^{3,9}$ & [24] \\
\hline
\end{tabular}

${ }^{1}$ donor based; ${ }^{2}$ isolated yield(s); ${ }^{3}$ not isolated; ${ }^{4}$ no product hydrolysis; ${ }^{5}$ donor added stepwise; ${ }^{6}$ thioglycoligase reaction; ${ }^{7} 20 \%(v / v)$ DMSO added; ${ }^{8} 15 \%(v / v)$ DMSO added $;{ }^{9} 5 \%(v / v)$ DMSO and 10 mM DTT added; ${ }^{10}$ Rme4 = K452M, N500S, I510N, N553S, W805R; ${ }^{11}$ RM34 = N500I, M547K, Y594F, N681S, K685R, N702Y, I716A, D746G, S860I; ${ }^{12}$ RMf125 = I485F, D746V, I762T, E787A, S889G. 
Table 6. Trans-glycosylation reactions catalyzed by mammalian, plant or mollusc GH20 $\beta$ - $N$-acetylhexosaminidases using pNP-activated carbohydrate donor substrates. New bonds are highlighted in bold.

\begin{tabular}{|c|c|c|c|c|c|c|c|c|c|}
\hline $\begin{array}{c}\text { Enzyme } \\
\text { (Organism) }\end{array}$ & $\begin{array}{l}\text { Acceptor } \\
(c[\mathrm{mM}])\end{array}$ & $\begin{array}{l}\text { Donor } \\
(c[\mathrm{mM}])\end{array}$ & $\begin{array}{l}\text { A:D } \\
\text { Ratio }\end{array}$ & Product(s) & $\mathrm{pH}$ & $\begin{array}{c}T \\
{\left[{ }^{\circ} \mathrm{C}\right]}\end{array}$ & $t[\mathrm{~h}]$ & $\begin{array}{l}\text { Yield } \\
\text { [\% }^{1}\end{array}$ & Ref. \\
\hline BtHex (Bos taurus) & $\begin{array}{c}\text { 2-amino-6-benzyl-2- } \\
\text { deoxy-Glc- } \beta \text {-S-Et (99/112) }\end{array}$ & $\begin{array}{l}\text { pNP-GlcNAc (52), } \\
\text { pNP-GalNAc (60) }\end{array}$ & $2: 1$ & $\begin{array}{l}\text { GlcNAc- } \beta-1,3-2 \text {-amino-6-benzyl-2-deoxy-Glc- } \beta \text {-S-Et (I), } \\
\text { GalNAc- } \beta-\mathbf{1}, \mathbf{3}-2 \text {-amino-6-benzyl-2-deoxy-Glc- } \beta \text {-S-Et (II) }\end{array}$ & $\begin{array}{l}6.8 \\
6.3\end{array}$ & 30 & $\begin{array}{l}50 \\
42\end{array}$ & 3.0 (I), 4.1 (II) ${ }^{2}$ & {$[53]$} \\
\hline $\begin{array}{l}\text { CeHex (Canavalia } \\
\text { ensiformis) }\end{array}$ & $\begin{array}{l}\text { Man- } \alpha-O-M e(579) \\
\text { Gal- } \beta-O-M e(579)\end{array}$ & pNP-GlcNAc (62) & 9:1 & $\begin{array}{l}\text { GlcNAc- } \beta-1,3-M a n-\alpha-O-M e(I) \\
\text { GlcNAc- } \beta-1,3-G a l-\beta-O-M e(I I)\end{array}$ & 6.5 & r.t. & 65 & $\begin{array}{l}16.5(\mathrm{I}), 14.0 \\
\quad(\mathrm{II}), 3\end{array}$ & [106] \\
\hline \multirow[t]{2}{*}{$\begin{array}{l}\text { CgHex (Chamelea } \\
\text { gallina) }\end{array}$} & $\begin{array}{l}\text { Gal- } \alpha-O-M e(515) \\
\text { Man- } \alpha-O-M e(515)\end{array}$ & $\begin{array}{l}\text { pNP-GlcNAc } \\
(50 / 100)\end{array}$ & $\begin{array}{c}10: 1 \\
5: 1\end{array}$ & $\begin{array}{l}\text { GlcNAc- } \beta-1,6-\text { Man- } \alpha-O-M e \\
\text { (I), GlcNAc- } \beta-1,6-\text { Gal- } \alpha-O-M e(I I)\end{array}$ & 5.8 & r.t. & 65 & 8 (I), $5.6(\mathrm{II})^{2,3}$ & [73] \\
\hline & Gal- $\beta-O-M e(515 / 644)$ & $\begin{array}{l}\text { pNP-GlcNAc (50), } \\
\text { pNP-GalNAc (73) }\end{array}$ & $\begin{array}{l}10: 1 \\
9: 1 \\
26: 1\end{array}$ & $\begin{array}{l}\text { GlcNAc- } \beta-\mathbf{1}, 3-G a l-\beta-O-M e(I), \\
\text { GlcNAc- } \beta-1,6-G a l-\beta-O-M e(I I), \\
\text { GalNAc- } \beta-\mathbf{1}, 3-G a l-\beta-O-M e(I I I)\end{array}$ & 8.5 & 37 & $\begin{array}{r}80 \\
120\end{array}$ & $\begin{array}{l}8.8(\mathrm{I}+\mathrm{II}), 6.2 \\
\text { (III) }^{2}\end{array}$ & [73] \\
\hline $\begin{array}{l}\text { VrHex }(\text { Vigna } \\
\quad \text { radiata })\end{array}$ & GlcNAc- $\beta-N-A c$ (138) & pNP-GlcNAc (14) & 10:1 & GlcNAc- $\beta-1,6-G l c N A c-\beta-N-A c$ & 6.5 & 37 & 8 & $12^{2,4}$ & [49] \\
\hline
\end{tabular}

${ }^{1}$ donor based; ${ }^{2}$ isolated yield(s); ${ }^{3} \%$ (v/v) DMF added; ${ }^{4}$ isolated as peracetylated derivate(s).

Table 7. Trans-glycosylation reactions catalyzed by GH20 $\beta-N$-acetylhexosaminidases using other activated carbohydrate donor substrates. New bonds are highlighted in bold.

\begin{tabular}{|c|c|c|c|c|c|c|c|c|c|}
\hline $\begin{array}{c}\text { Enzyme } \\
\text { (Organism) }\end{array}$ & $\begin{array}{l}\text { Acceptor } \\
(c[\mathrm{mM}])\end{array}$ & $\begin{array}{l}\text { Donor } \\
(c[\mathrm{mM}])\end{array}$ & $\begin{array}{l}\text { A:D } \\
\text { Ratio }\end{array}$ & Product(s) & $\mathrm{pH}$ & $\begin{array}{c}T \\
{\left[{ }^{\circ} \mathrm{C}\right]} \\
\end{array}$ & $t[\mathrm{~h}]$ & $\begin{array}{l}\text { Yield } \\
{[\%]^{1}}\end{array}$ & Ref. \\
\hline \multirow[t]{2}{*}{ AoHex $(\text { A. oryzae })^{2}$} & $\begin{array}{c}\text { pNP-Glc (100), pNP-Gal } \\
(100)\end{array}$ & GlcNAc- $\beta-O-N P y(50)$ & 2:1 & $\begin{array}{l}\text { GlcNAc- } \beta-1,4-G l c-\beta-O-p N P(I) \\
\text { GlcNAc- } \beta-1,4-G a l-\beta-O-p N P \text { (II) }\end{array}$ & 6.0 & 25 & 0.75 & $8.6(\mathrm{I}), 6.1$ (II) ${ }^{3}$ & [72] \\
\hline & $\begin{array}{c}\text { Glc, Gal, Man, } \\
\text { 2-deoxy-Glc, GlcNAc, } \\
\text { GlcNPr, GlcNFo (1100 } \\
\text { each), maltose, Lac (570 } \\
\text { each) }\end{array}$ & GlcNAc- $\beta-O-P h(33)$ & $\begin{array}{l}33: 1 \\
17: 1\end{array}$ & 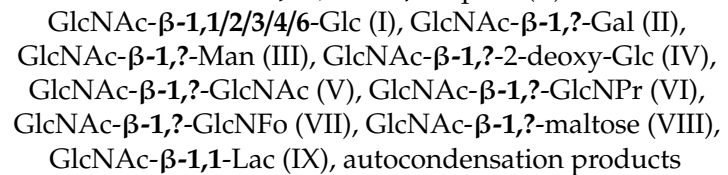 & 4.5 & 37 & n.a. & $\begin{array}{c}36 \text { (I), } 38 \text { (II), } 3 \\
\text { (III), } 10 \text { (IV), } \\
18 \text { (V), } 11 \text { (VI), } \\
22 \text { (VII), } 24 \\
\text { (VIII), } 31 \text { (IX) } 5\end{array}$ & [71] \\
\hline $\mathrm{CgHex}(C$. gallina $)$ & Gal- $\alpha-O-M e(257)$ & GalNAc- $\alpha-O-o N P(10)$ & $26: 1$ & GlcNAc- $\alpha-1,3-G a l-\alpha-O-M e$ & 5.8 & 37 & 120 & $6.3^{3}$ & [73] \\
\hline \multirow[t]{2}{*}{$\begin{array}{l}\text { TfHex (T. flavus } \\
\text { CCF 2686) }\end{array}$} & GlcNAc (306) & $\begin{array}{c}\text { 4-deoxy-GlcNAc- } \beta-O-P h \\
\text { (75) }\end{array}$ & $4: 1$ & $\begin{array}{c}\text { 4-deoxy-GlcNAc- } \beta-\mathbf{1}, \mathbf{4}-G l c N A c(\mathrm{I}) \\
\text { 4-deoxy-GlcNAc- } \beta-\mathbf{1}, \mathbf{6}-G l c N A c \text { (II), } \\
\text { 4-deoxy-GlcNAc- } \beta-\mathbf{- 1 , 6 - 4 - d e o x y - G l c N A c - \beta - O - P h ~ ( I I I ) ~}\end{array}$ & 5.0 & 35 & 5.5 & $\begin{array}{l}7 \text { (I), } 6 \text { (III), } \\
14(\mathrm{IIII})^{3}\end{array}$ & [65] \\
\hline & GalNAc- $\beta-N_{3}(400)$ & GlcNAc- $\beta-\mathrm{N}_{3}(100)$ & $4: 1$ & GlcNAc- $\beta-1,6-$-GalNAc- $\beta-N_{3}$ & 5.0 & 35 & 3.5 & $22^{3}$ & [66] \\
\hline
\end{tabular}

${ }^{1}$ donor based $;{ }^{2}$ purified from $\beta$-galactosidase preparation (grade XI, Sigma-Aldrich); ${ }^{3}$ isolated yield(s); ${ }^{4}$ purified from Taka-diastase (Sankyo); ${ }^{5}$ products not isolated. 


\subsection{3. $N, N^{\prime}$-Diacetylchitobiose and Chitooligomers as Donor}

Potentially more sustainable alternatives to the synthetic donors include the fully $N$-acetylated chitooligomers $\left((\mathrm{GlcNAc})_{\mathrm{n}}\right)$ and specifically $N, N^{\prime}$-diacetylchitobiose (Figure 3: (GlcNAc) $)_{2}$; Tables 2 and 8 ). These donor substrates are derived from the natural biopolymer chitin and the byproduct of the enzyme catalyzed trans-glycosylation reaction, GlcNAc, is completely biocompatible. Already 30 years ago, it was shown that the hexosaminidase from Nocardia orientalis (NoHex; now known as Amycolatopsis orientalis) is able to synthesize (GlcNAc) ${ }_{3}$ and GlcNAc- $\beta-1,6-G l c N A c$ from $(G l c N A c)_{2}$ in an auto-condensation reaction (Table 2) [60]. The first example utilizing (GlcNAc) $3-4$ followed three years later in a study of the hexosaminidase from Vibrio sp. (VsHex, Table 2), although the exact products were not identified [67]. Bacterial as well as fungal enzymes, as shown for AoHex, can utilize $(\mathrm{GlcNAc})_{\mathrm{n}}$ as donor $[55,57]$. However, the high price for specific chitooligomers and (GlcNAc) 2 $\left(3,150,000 € / \mathrm{kg}(1,337,000 € / \mathrm{mol})\right.$ for $(\mathrm{GlcNAc})_{2}$ at Carbosynth) [107], i.e., even more expensive than UDP-GlcNAc, currently prevents their use as substrates in any industrial process. An alternative process may involve development of safe, cost-efficient extraction of chitooligomers from shellfish waste as outlined previously [8].

\subsubsection{Oxazoline-Derivates as Activated Donors}

Recently, the oxazoline derivative of GlcNAc (Figure 3: Glc-oxa) has been demonstrated to work as donor molecule for GH20-catalyzed synthesis of the HMO-precursor molecule lacto-N-triose II (LNT II, Figure 1) using a mutant of the enzyme Hex1 [108], which was previously discovered in a metagenomic library [109]. Glc-oxa is the natural intermediate of a GH20 reaction and can easily be synthesized using high temperature and alkali [110]. However, oxazolines are unstable at acidic conditions and less useful, eg, in fungal hexosaminidase reactions having acidic pH optima [64]. Nevertheless, the ability of bacterial hexosaminidases with neutral or alkaline $\mathrm{pH}$ optima to utilize Glc-oxa as donor for trans-glycosylation reactions as demonstrated (Table 9) $[24,101,105,108,111]$ together with its comparably good price per mole $(1,710,000 € / \mathrm{kg}(347,000 € / \mathrm{mol})$ for Glc-oxa at Carbosynth [112]) may increase the popularity of Glc-oxa as donor molecule for such enzymatic synthesis reactions in the future. The fact that there are no byproducts from trans-glycosylation reactions using Glc-oxa and the option of conducting Glc-oxa synthesis under mild conditions $[113,114]$ with enzymatic trans-glycosylation in a cascade fashion [108] might lead to industrialization of GH20-catalyzed trans-glycosylation with Glc-oxa donors.

\subsection{Acceptor:Donor Ratio}

Another general strategy to increase trans-glycosylation yields is to increase the acceptor:donor ratio (A:D ratio). This approach has proven successful for many trans-glycosylation reactions involving different enzymes as reviewed in [42]. The rationale of this strategy is the direct dependency of the ratio between trans-glycosylation rate and hydrolysis rate on the acceptor concentration [42]. The method has also been widely applied for GH20-catalyzed trans-glycosylation reactions (Tables 1 and 3-10). For carbohydrate acceptor substrates, the A:D ratio is somewhat limited by substrate solubility, but A:D ratios of up to 100:1 have been reported (Tables 3 and 5) [51,83]. Higher ratios of up to 910:1 have been achieved with non-carbohydrate acceptors like alcohols (Table 10) [71], which are usually highly soluble. However, apart from the auto-condensation reactions, for which the A:D ratio is always considered 1:1 (Table 2), a few examples of GH20 trans-glycosylation reactions using an A:D ratio of 1:1 also exist (Tables 1, 3, 4, 9 and 10) [19,23,24,46,52,53,64,88,90,101,115,116]. Most noteworthy in this context is the recently reported synthesis of LNT II where an isolated yield of $86 \%(281 \mathrm{~g} / \mathrm{L})$ was achieved in a reaction using Lac and Glc-oxa in a ratio of 1:1 at a concentration of $600 \mathrm{mM}$ each (Table 9) [100]. 
Table 8. Trans-glycosylation reactions catalyzed by GH20 $\beta$-N-acetylhexosaminidases using chitobiose donor substrates. New bonds are highlighted in bold.

\begin{tabular}{|c|c|c|c|c|c|c|c|c|c|}
\hline $\begin{array}{c}\text { Enzyme } \\
\text { (Organism) }\end{array}$ & $\begin{array}{l}\text { Acceptor } \\
(c[\mathrm{mM}])\end{array}$ & $\begin{array}{l}\text { Donor } \\
(c[\mathrm{mM}])\end{array}$ & $\begin{array}{l}\text { A:D } \\
\text { Ratio }\end{array}$ & Product(s) & $\mathrm{pH}$ & $T\left[{ }^{\circ} \mathrm{C}\right]$ & $t[\mathrm{~h}]$ & $\begin{array}{l}\text { Yield } \\
\text { [\% }^{1}\end{array}$ & Ref. \\
\hline \multirow[t]{2}{*}{$\begin{array}{c}\text { Hex1 } \\
\text { (metagenomic) }\end{array}$} & Lac $(500)$ & $\begin{array}{c}(\text { GlcNAc })_{2} \\
\quad(100)\end{array}$ & $5: 1$ & LNT II $(\beta-1,3)(\mathrm{I})$, other regioisomers $(\beta-1, ?)$ (II) & 8.0 & 25 & 2 & 2 (I), 0.1 (II) ${ }^{2}$ & [109] \\
\hline & Lac $(500)$ & $\begin{array}{c}(\text { GlcNAc })_{2} \\
\quad(100)\end{array}$ & $5: 1$ & LNT II $(\beta-1,3)(\mathrm{I})$, other regioisomers $(\beta-1, ?)$ (II) & 7.0 & 25 & 8.5 & $5.7(\mathrm{I}+\mathrm{II})^{2}$ & [117] \\
\hline a) GTDDA $^{8}$ & Lac (500) & $\begin{array}{l}(\mathrm{GlcNAc})_{2} \\
\quad(100)\end{array}$ & $5: 1$ & LNT II $(\beta-1,3)(\mathrm{I})$, other regioisomers $(\beta-1, ?)$ (II) & 7.0 & 25 & 24 & $23.3(\mathrm{I}+\mathrm{II})^{2}$ & [117] \\
\hline b) GTEPG $^{8}$ & Lac $(500)$ & $\begin{array}{l}(\mathrm{GlcNAc})_{2} \\
\quad(100)\end{array}$ & $5: 1$ & LNT II $(\beta-1,3)(\mathrm{I})$, other regioisomers $(\beta-1, ?)$ (II) & 7.0 & 25 & 47 & $29.5(\mathrm{I}+\mathrm{II})^{2}$ & {$[117]$} \\
\hline \multirow{5}{*}{$\begin{array}{l}\text { Hex2 } \\
\text { (metagenomic) } \\
\text { NoHex }(N . \\
\text { orientalis } \\
\left.\text { IFO12806 }{ }^{3}\right)\end{array}$} & Lac $(500)$ & $\begin{array}{c}(\text { GlcNAc })_{2} \\
\quad(100)\end{array}$ & $5: 1$ & LNT II $(\beta-1,3)(\mathrm{I})$, other regioisomers $(\beta-1, ?)$ (II) & 6.0 & 25 & 2 & 8.3 (I), 9.1 (II) ${ }^{2}$ & [109] \\
\hline & $\begin{array}{l}\text { pNP-LacNAc } \\
(155)\end{array}$ & $\begin{array}{l}(\mathrm{GlcNAc})_{2} \\
\quad(730)\end{array}$ & $1: 5$ & $\begin{array}{c}\text { GlcNAc-LacNAc- } \beta-O-p N P(\text { three regiosomers: } \\
\qquad-\mathbf{1 , 3} \text { and } \boldsymbol{\beta}-\mathbf{1 , 6})\end{array}$ & 5.0 & 40 & 12 & $2.9-7.4^{4,5}$ & [118] \\
\hline & $\begin{array}{l}\text { Lac- } \beta-O-M e \\
\quad(737)\end{array}$ & $\begin{array}{l}(\mathrm{GlcNAc})_{2} \\
\quad(324)\end{array}$ & $2: 1$ & $\begin{array}{c}\text { LNT II- } \beta-O-M e(\beta-1,3)(\mathrm{I}) \\
\text { GlcNAc- } \beta-1,6-\text { Lac- } \beta-O-M e(I I) \\
\text { Gal- } \beta-1,4-(\text { GlcNAc- } \beta-1,6) \text { Glc- } \beta-O-M e(I I I)\end{array}$ & 5.0 & 40 & 20 & $17(\mathrm{I}+\mathrm{II}+\mathrm{III})^{4}$ & [119] \\
\hline & pNP-Lac (168) & $\begin{array}{l}(\mathrm{GlcNAc})_{2} \\
\quad(317)\end{array}$ & $1: 2$ & $\begin{array}{c}\text { LNT II- } \beta-O-p N P(\beta-1,3)(\mathrm{I}) \\
\text { GlcNAc- } \beta-1,6-\text { Lac- } \beta-O-p N P \text { (II), } \\
\text { Gal- } \beta-1,4-(\text { GlcNAc- } \beta-1,6) \text { Glc- } \beta-O-p N P \text { (III) }\end{array}$ & 5.0 & 40 & 12 & $2.4(\mathrm{I}+\mathrm{II}+\mathrm{III})^{4,6}$ & [119] \\
\hline & $\begin{array}{l}\text { Gal- } \beta-1,3- \\
\text { GalNAc- } \alpha-O- \\
\operatorname{pNP}(23)\end{array}$ & $\begin{array}{c}(\mathrm{GlcNAc})_{2} \\
\quad(239)\end{array}$ & $1: 10$ & $\begin{array}{l}\text { Gal- } \beta-1,3-(G l c N A c-\beta-1,6-) \text { GalNAc- } \alpha-O-p N P(I), \\
\text { GlcNAc- } \beta-1,6-G a l-\beta-1,3-G a l N A c-\alpha-O-p N P(I I), \\
\text { GlcNAc- } \beta-1,3-G a l-\beta-1,3-G a l N A c-\alpha-O-p N P(I I I), \\
\text { Gal- } \beta-1,3-(G 1 c N A c-\beta-1,6-) \text { GalNAc- } \beta-O-p N P(I V), \\
\text { GlcNAc- } \beta-1,6-G a l-\beta-1,3-G a l N A c-\beta-O-p N P(V), \\
\text { GlcNAc- } \beta-1,3-G a l-\beta-1,3-G a l N A c-\beta-O-p N P(V I),\end{array}$ & 5.0 & 40 & 12 & $\begin{array}{c}14(\mathrm{I}+\mathrm{II}+\mathrm{III}), 8 \\
(\mathrm{IV}+\mathrm{V}+\mathrm{VI})^{7}\end{array}$ & [120] \\
\hline
\end{tabular}

$\alpha$-cyclodextrin influenced regioselectivity; ${ }^{8}$ specified loop sequence inserted in between W354 and R355 of Hex1. 
Table 9. Trans-glycosylation reactions catalyzed by GH20 enzymes using oxazoline-activated carbohydrate donor substrates. New bonds are highlighted in bold.

\begin{tabular}{|c|c|c|c|c|c|c|c|c|c|}
\hline $\begin{array}{c}\text { Enzyme } \\
\text { (Organism) }\end{array}$ & $\begin{array}{l}\text { Acceptor } \\
(c[\mathrm{mM}])\end{array}$ & $\begin{array}{l}\text { Donor } \\
(c[\mathrm{mM}])\end{array}$ & $\begin{array}{l}\text { A:D } \\
\text { Ratio }\end{array}$ & Product(s) & $\mathrm{pH}$ & $T\left[{ }^{\circ} \mathrm{C}\right]$ & $t[\mathrm{~h}]$ & $\begin{array}{l}\text { Yield } \\
{[\%]^{1}}\end{array}$ & Ref. \\
\hline $\begin{array}{l}\text { BbhI (B. bifidum } \\
\text { JCM1254) }\end{array}$ & Lac (600) & Glc-oxa (60) & $10: 1$ & LNT II $(\beta-1,3)$ & 7.5 & 37 & 2 & $58^{2}$ & [101] \\
\hline \multirow[t]{2}{*}{ a) D746E } & Lac (600) & Glc-oxa (60) & 10:1 & LNT II $(\boldsymbol{\beta}-\mathbf{1}, \mathbf{3})$ & 7.5 & 37 & 0.5 & $85^{2}$ & {$[101]$} \\
\hline & Lac (600) & Glc-oxa (600) & $1: 1$ & LNT II $(\beta-1,3)$ & 7.5 & 37 & 0.5 & $86^{3}$ & [101] \\
\hline b) $\mathrm{D} 746 \mathrm{~A}$ & Lac (600) & Glc-oxa (60) & $10: 1$ & LNT II $(\boldsymbol{\beta}-\mathbf{1}, \mathbf{3})$ & 7.5 & 37 & 5 & $80^{2,4}$ & [101] \\
\hline c) $\mathrm{D} 746 \mathrm{Q}$ & Lac (600) & Glc-oxa (60) & $10: 1$ & LNT II $(\beta-1,3)$ & 7.5 & 37 & 8 & $16^{2,4}$ & [101] \\
\hline d) $Y 827 \mathrm{~F}$ & Lac (600) & Glc-oxa (60) & $10: 1$ & LNT II $(\beta-1,3)$ & 7.5 & 37 & 2.5 & $80^{2}$ & [101] \\
\hline $\begin{array}{l}\text { Hex1GTEPG }^{9} \\
\text { (metagenomic) }\end{array}$ & Lac $(500)$ & Glc-oxa (100) & $5: 1$ & $\begin{array}{c}\text { LNT II (I) }(\beta-1,3) \text {, three } \\
\text { unidentified regioisomers }(\beta-1, ?) \\
\text { (II) }\end{array}$ & 8.0 & 25 & 6.5 & $>25(\mathrm{I}+\mathrm{II})^{2}$ & [108] \\
\hline $\begin{array}{l}\text { LnbB (B. bifidum } \\
\text { JCM 1254) }\end{array}$ & Lac (600) & $\begin{array}{l}\text { Gal- } \beta-1,3-\text { Glc-oxa } \\
\text { (LNB-oxa) (12) }\end{array}$ & $50: 1$ & $\operatorname{LNT}(\beta-1,3)$ & 7.5 & 37 & 0.1 & $67^{2}$ & [105] \\
\hline a) D320E & Lac (600) & LNB-oxa (12) & $50: 1$ & LNT $(\beta-1,3)$ & 7.5 & 37 & 1 & $29^{2,4}$ & [105] \\
\hline b) D320A & Lac (600) & LNB-oxa (12) & $50: 1$ & LNT $(\beta-1,3)$ & 7.5 & 37 & 21 & $13^{2,4}$ & [105] \\
\hline c) $\mathrm{Y} 419 \mathrm{~F}$ & Lac (600) & LNB-oxa (12) & $50: 1$ & LNT $(\beta-1,3)$ & 7.5 & 37 & 2.5 & $37^{2}$ & [105] \\
\hline $\begin{array}{c}\text { PhNah20A } \\
(\text { Paraglaciecola } \\
\left.\text { hydrolytica } 566^{\mathrm{T}}\right)\end{array}$ & Lac (200) & Glc-oxa (100) & $2: 1$ & $\begin{array}{c}\text { Lac- } \beta-\mathbf{1}, \mathbf{1}-\boldsymbol{\beta}-\text { GlcNAc }(\mathrm{I}), \text { LNT II } \\
(\boldsymbol{\beta}-\mathbf{1}, \mathbf{3})(\mathrm{II}), \text { unidentified } \\
\text { regioisomer }(\boldsymbol{\beta}-\mathbf{1}, \mathbf{?})(\mathrm{III})^{7}\end{array}$ & 8.0 & 37 & 2 & $3.8(\mathrm{I}+\mathrm{II}+\mathrm{III})$ & {$[111]$} \\
\hline SpHex (S. plicatus) & Lac (200) & Glc-oxa (100) & $2: 1$ & $\begin{array}{c}\text { LNT II }(\beta-1,3), \text { unidentified } \\
\text { regioisomer }(\beta-1, ?)\end{array}$ & 8.0 & 37 & 1 & n.a. ${ }^{2,8}$ & [111] \\
\hline a) $\mathrm{E} 314 \mathrm{~A}$ & $\begin{array}{c}\text { 3/4/6-S-GlcNAc- } \beta-O-p N P, \\
\text { 3/4-S-GalNAc- } \beta-O-p N P \\
\text { 4-S-ManNAc- } \beta-O-p N P \\
\text { 4-S-Man- } \beta \text {-O-pNP (5 each) }\end{array}$ & Glc-oxa (30) & $1: 6$ & Different thioglycosides $(\beta-S)^{5,6}$ & 7.0 & 37 & $<1$ & $>99^{2}$ & [24] \\
\hline
\end{tabular}

${ }^{1}$ donor based; ${ }^{2}$ not isolated; ${ }^{3}$ isolated yield; ${ }^{4}$ no product hydrolysis; ${ }^{5}$ thioglycoligase reaction; ${ }^{6} 5 \%(v / v)$ DMSO and 10 mM DTT added; ${ }^{7}$ probably Gal- $\beta-1,4-($ GlcNAc- $\beta$-1,2-)Glc or Gal- $\beta-1,4-($ GlcNAc- $\beta-1,3-)$ Glc; $^{8}$ only analyzed by TLC; ${ }^{9}$ specified loop sequence inserted in between W354 and R355 of Hex1. 
Table 10. Synthetic trans-glycosylation reactions using non-carbohydrate acceptors catalyzed by GH20 $\beta$ - $N$-acetyl hexosaminidases. New bonds are highlighted in bold.

\begin{tabular}{|c|c|c|c|c|c|c|c|c|c|}
\hline $\begin{array}{c}\text { Enzyme } \\
\text { (Organism) }\end{array}$ & $\begin{array}{l}\text { Acceptor } \\
(c[\mathrm{mM}])\end{array}$ & $\begin{array}{c}\text { Donor } \\
(c[\mathrm{mM}])\end{array}$ & $\begin{array}{l}\text { A:D } \\
\text { ratio }\end{array}$ & Product(s) & $\mathrm{pH}$ & $T\left[{ }^{\circ} \mathrm{C}\right]$ & $t[\mathrm{~h}]$ & $\begin{array}{l}\text { Yield } \\
{[\%]^{1}}\end{array}$ & Ref. \\
\hline $\begin{array}{l}\text { AnHex (A. niger } \\
\text { CCIM K2) }\end{array}$ & $t$-Boc-Ser, $t$-Boc-Thr (1130) & $\alpha$-GalNAc (1130) & $1: 1$ & $\begin{array}{l}\text { GalNAc- } \alpha-O-S e r(I), \\
\text { GalNAc- } \alpha-O \text {-Thr (II) }\end{array}$ & 4.8 & 35 & 168 & 7.4 (I), 3.6 (II) $)^{2,3}$ & [23] \\
\hline AoHex $(\text { A. oryzae })^{4}$ & $\begin{array}{l}\text { Elymoclavine (95), } \\
\text { Chanoclavine (95) }\end{array}$ & pNP-GalNAc (44) & 2:1 & $\begin{array}{l}\text { GalNAc- } \beta \text {-O-elymoclavine (I), } \\
\text { GalNAc- } \beta \text { - } O \text {-chanoclavine(II) }\end{array}$ & 4.5 & 33 & 60,28 & 15 (I), 4.8 (II) ${ }^{2,5}$ & [21] \\
\hline 4 & Ergometrine (95) & pNP-GalNAc (44) & $2: 1$ & GalNAc- $\beta$-O-ergometrine & 4.5 & 33 & 26 & $12^{2}$ & [21] \\
\hline 4 & $\begin{array}{l}\text { Elymoclavine (95), } \\
\text { Chanoclavine (95) }\end{array}$ & pNP-GlcNAc (44) & $2: 1$ & $\begin{array}{l}\text { GlcNAc- } \beta \text { - } O \text {-elymoclavine (I), } \\
\text { GlcNAc- } \beta \text { - } O \text {-chanoclavine (II) }\end{array}$ & 4.5 & 28 & 70,52 & $13.4(\mathrm{I}), 8.1(\mathrm{II})^{2}$ & [21] \\
\hline 4 & $\begin{array}{c}\text { Ergometrine/Ergometrinine } \\
\text { (95) }\end{array}$ & pNP-GlcNAc (44) & $2: 1$ & $\begin{array}{l}\text { GlcNAc- } \beta \text {-O-ergometrine, } \\
\text { GlcNAc- } \beta \text { - } O \text {-ergometrinine }\end{array}$ & 4.5 & 28 & 52 & $10.4^{2}$ & [21] \\
\hline 6 & Sphingosine (1670) & $\begin{array}{c}\text { GlcNAc (180), } \\
\text { Et-GlcNAc (321), } \\
\text { Pr-GlcNAc (683) }\end{array}$ & $1: 1$ & $\begin{array}{c}\text { GlcNAc- } \beta \text {-O-sphingosine (I), } \\
(\text { GlcNAc) })_{2}(\boldsymbol{\beta - 1 , 4 )} \text { (II), }(\text { GlcNAc) } \\
(\boldsymbol{\beta - 1 , 4 )} \text { (III) }\end{array}$ & 7.0 & 40 & 36 & $\begin{array}{l}44 \text { (I), } 14 \text { (II), } 8 \\
\text { (III) }{ }^{2}\end{array}$ & $\begin{array}{l}{[115} \\
116]\end{array}$ \\
\hline 4 & $\begin{array}{c}\text { 1,3,5-tris(hydroxyethyl) } \\
\text { cyanuric acid (Thca) (77), } \\
\text { 1,4-benzenedimethanol (Bm) } \\
\text { (145) }\end{array}$ & $\begin{array}{c}\text { pNP-GlcNAc }(58+2 \\
\times 29)^{8}\end{array}$ & 1:1, 3:1 & $\begin{array}{l}\text { GlcNAc- } \beta-O-T h c a(\mathrm{I}) \\
\text { GlcNAc- } \beta-O-B m \text { (II) }\end{array}$ & 5.0 & n.a. & $>120$ & 29 (I), 12 (II) ${ }^{2}$ & [19] \\
\hline 9 & $\begin{array}{l}\text { Several alcohols, ethylene } \\
\text { glycol, glycerol, erythritol, } \\
\text { three alditols (910 each) }\end{array}$ & Ph-GlcNAc (1) & 910:1 & $\begin{array}{l}\text { Alkyl-glycosides }(\beta-O)(\mathrm{I})^{10} \\
\text { autocondensation products }\end{array}$ & 4.5 & 37 & 0.6 & $27-86$ (I) ${ }^{11}$ & [71] \\
\hline \multirow[t]{2}{*}{$\begin{array}{l}\text { AoHex (A. oryzae } \\
\text { CCF 1066) }\end{array}$} & Thiamine (370) & $\begin{array}{c}\text { pNP-GlcNAc }(125+ \\
74+52)^{8}\end{array}$ & $3: 1$ & GlcNAc- $\beta$ - $O$-thiamine & 5.0 & 37 & 11 & $3.5^{2}$ & [22] \\
\hline & Pyridoxine (195) & $\begin{array}{c}\text { pNP-GlcNAc } \\
\times 9)^{8}\end{array}$ & $2: 1$ & $\begin{array}{l}\text { GlcNAc- } \beta-O-4 a-p y r i d o x i n e ~(I), \\
\text { GlcNAc- } \beta-O \text {-5a-pyridoxine (II) }\end{array}$ & 5.0 & 24 & 3 & $13.5(\mathrm{I}+\mathrm{II})^{2}$ & [121] \\
\hline $\begin{array}{l}\text { BbhI (B. bifidum } \\
\text { JCM1254) }\end{array}$ & $\begin{array}{c}\text { Different alcohols }(20 \%(v / v) \\
\text { each) }\end{array}$ & LNT (1.3) & n.a. & n.d. & 6.0 & 40 & n.a. & n.a. ${ }^{11}$ & [51] \\
\hline $\begin{array}{l}\text { NoHex (Nocardia } \\
\text { orientalis IFO12806 } \\
13 \text { ) }\end{array}$ & $\begin{array}{l}\text { 1,6-hexanediol (Hx) (50), } \\
\text { triethylene glycol (Doo) (51) }\end{array}$ & $(\mathrm{GlcNAc})_{4}(100)$ & $1: 2$ & $\begin{array}{c}\text { GlcNAc- } \beta-O-H x(\mathrm{I}) / \text { Doo } \\
\text { (II)-O- } \beta-G l c N A c \\
\text { GlcNAc- } \beta-O-H x(\mathrm{III}) / \text { Doo (IV), } \\
(\mathrm{GlcNAc})_{2}-\boldsymbol{\beta}-\mathrm{O}-\mathrm{Hx}(\mathrm{V}) / \text { Doo (VI) } \\
(\boldsymbol{\beta}-\mathbf{1}, \mathbf{4})\end{array}$ & 6.7 & 40 & 45 & $\begin{array}{c}10.5 \text { (I), } 22.6 \text { (III), } \\
1.4 \text { (V); } 4.7 \text { (II), } \\
69.0(\mathrm{IV}), 0.75 \\
\text { (VI) }^{2}\end{array}$ & [20] \\
\hline $\begin{array}{l}\text { PoHex (Penicillium } \\
\text { oxalicum) }\end{array}$ & $\begin{array}{l}\text { Cyclohexanol (Chx) (327), } \\
\text { coniferyl alcohol (Con) (200) }\end{array}$ & $\begin{array}{c}\text { pNP-GalNAc }(30+2 \\
\times 15)^{8}\end{array}$ & $11: 1,7: 1$ & $\begin{array}{c}\text { GalNAc- } \beta-O-C h x(\mathrm{I}), \\
\text { GalNAc- } \beta-1,6-\mathrm{C} \text { (II), } \\
\text { GalNAc- } \beta-O-C o n(\mathrm{III}), \\
\text { GalNAc- } \beta-\mathbf{1 , 6 - I I I ~ ( I V ) ~}{ }^{14}\end{array}$ & 5.0 & 35 & 7 & $\begin{array}{l}21 \text { (I), } 5 \text { (II), } 20 \\
\text { (III), } 3 \text { (IV) }{ }^{2}\end{array}$ & [92] \\
\hline
\end{tabular}


Table 10. Cont.

\begin{tabular}{|c|c|c|c|c|c|c|c|c|c|}
\hline $\begin{array}{c}\text { Enzyme } \\
\text { (Organism) }\end{array}$ & $\begin{array}{l}\text { Acceptor } \\
(c[\mathrm{mM}])\end{array}$ & $\begin{array}{l}\text { Donor } \\
(c[\mathrm{mM}])\end{array}$ & $\begin{array}{l}\text { A:D } \\
\text { ratio }\end{array}$ & Product(s) & $\mathrm{pH}$ & $T\left[{ }^{\circ} \mathrm{C}\right]$ & $t[\mathrm{~h}]$ & $\begin{array}{l}\text { Yield } \\
\text { [\% }^{1}\end{array}$ & Ref. \\
\hline \multirow{5}{*}{$\begin{array}{l}\text { PoHex (P. oxalicum } \\
\text { IFO 5748) }\end{array}$} & $t$-butanol & pNP-GalNAc (30) & n.a. & Not identified & 5.0 & 35 & 24 & $0.7^{11}$ & [92] \\
\hline & Myo-inositol (500) & $\begin{array}{c}\text { pNP-GalNAc }(30+2 \\
\times 30)^{8,19}\end{array}$ & $17: 1$ & $\begin{array}{l}\text { GalNAc- } \beta \text {-O-myo-inositol } \\
\text { regioisomers I and II }\end{array}$ & 5.0 & 35 & 7 & $3(\mathrm{I}+\mathrm{II})^{2}$ & [92] \\
\hline & Pyridine-3-aldoxime & pNP-GalNAc (30) ${ }^{19}$ & n.a. & Not identified & 5.0 & 35 & 24 & $<1^{11}$ & [92] \\
\hline & $\begin{array}{c}\text { Several alcohols }(22 \%(v / v) \\
\text { each) }\end{array}$ & $(\text { GlcNAc })_{3}(16)$ & $236: 1-88: 1$ & GlcNAc- $\beta-O$-alkyl & 4.5 & 37 & 1.5 & n.a. ${ }^{12}$ & {$[122]$} \\
\hline & $\begin{array}{c}\text { 6-Benzyloxyhexan-1-ol (Bhx) } \\
(955)(20 \%(v / v))\end{array}$ & $(\mathrm{GlcNAc})_{3}(16)$ & $60: 1$ & GlcNAc- $\beta-O-B h x$ & 4.5 & 37 & 1 & $32^{2}$ & {$[122]$} \\
\hline \multirow[t]{2}{*}{$\begin{array}{l}\text { SmHex (Serratia } \\
\text { marcescens YS-1) }\end{array}$} & $\begin{array}{l}\text { Several alcohols, diols and } \\
\text { one triol }(8 \%(v / v) \text { each })\end{array}$ & $(\text { GlcNAc })_{2}(94)$ & $21: 1-8: 1$ & $\begin{array}{l}(\text { GlcNAc })_{3}(\beta-1,4)(\mathrm{I}) \\
\text { GlcNAc- } \beta-O-\text {-alkyl (II) }\end{array}$ & 6.0 & 40 & 24 & $\begin{array}{c}15.3-65.9(\mathrm{II}) \\
2.2-26.7 \text { (I) }\end{array}$ & [62] \\
\hline & $\begin{array}{c}\text { Several alditols }(8 \%(w / v) \\
\text { each) }\end{array}$ & $(\mathrm{GlcNAc})_{2}(94)$ & $6: 1-3: 1$ & $\begin{array}{c}(\operatorname{GlcNAc})_{3}(\beta-1, \mathbf{4})(\mathrm{I}) \\
\text { GlcNAc- } \beta-O \text {-aldityl (II) }\end{array}$ & 6.0 & 40 & 24 & $\begin{array}{l}1.62-11.0(\mathrm{II}) \\
8.18-10.4(\mathrm{I})\end{array}$ & [62] \\
\hline \multirow[t]{4}{*}{$\begin{array}{l}\text { SpHexE314A } \\
\text { (Streptomyces } \\
\text { plicatus) }\end{array}$} & $\begin{array}{l}\text { 2,4-dinitrophenol (Dnp), } \\
\text { pNP, phenol (20 each), } \\
\text { 2-chlorophenol (Cp) (7) }\end{array}$ & Glc-oxa (5) 20 & $4: 1,1: 1$ & 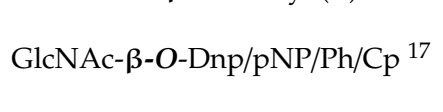 & 8.0 & 37 & $<1$ & $>99^{11}$ & [24] \\
\hline & Et-Cys & pNP-GlcNAc & n.a. & GlcNAc- $\beta$-S-Cys ${ }^{18}$ & n.a. & n.a. & n.a. & n.a. & [24] \\
\hline & $\begin{array}{c}\text { Synuclein model peptides: } \\
\text { VVCGV (0.002), AGCIA } \\
(0.002)\end{array}$ & pNP-GlcNAc (10) ${ }^{21}$ & $1: 5000$ & $\begin{array}{l}\text { GlcNAc- } \beta \text {-S-peptide conjugates } \\
\quad(\text { VVCGV (I), AGCIA (II)) })^{18}\end{array}$ & 7.0 & 37 & $<1$ & 44 (I), 30 (II) ${ }^{11}$ & [24] \\
\hline & $\begin{array}{l}\text { Tau protein }(244-441) \text { C } 301 S \\
\text { C322S S400C (0.16) }\end{array}$ & pNP-GlcNAc (6.5) & $1: 50$ & GlcNAc- $\beta$-S-Tau conjugate ${ }^{18}$ & 7.0 & 37 & 3 & $44^{11}$ & [24] \\
\hline
\end{tabular}

${ }^{1}$ donor based; ${ }^{2}$ isolated yield(s); ${ }^{3}$ isolated as deprotected conjugates; ${ }^{4}$ purified from $\beta$-galactosidase preparation (grade XI, Sigma-Aldrich); ${ }^{5} 2 \%$ diglycoside observed; ${ }^{6}$ enzyme source not specified; ${ }^{7}$ carried out in a plasticized glass phase: $10 \% \mathrm{H}_{2} \mathrm{O}, 5 \% \mathrm{EtOH}, 5 \% n$-PrOH; ${ }^{8}$ donor added stepwise; ${ }^{9}$ isolated from Taka-diastase (Sankyo); ${ }^{10} t$-butanol gave no product; ${ }^{11}$ not isolated; ${ }^{12}$ only detected by TLC; ${ }^{13}$ or Amycolatopsis orientalis IFO12806T; ${ }^{14}$ only aliphatic OH of coniferyl alcohol glycosylated; ${ }^{15}$ 1,3-butanediol and 1,2,4-butanetriol gave no product, but enhanced chitotriose formation; ${ }^{16}$ no product formation with dulcitol; ${ }^{17}$ Phenyl- and 2-chlorophenylglycosides were cleaved overnight, the other two were stable; ${ }^{18}$ thioglycoligase reaction; ${ }^{19} 30 \%(v / v)$ MeCN added; ${ }^{20}$ 5\% (v/v) DMSO added; ${ }^{21} 10 \mathrm{mM}$ DTT added. 
A few examples of successful reactions with A:D ratios $<1$ (as low as 1:10) have been reported (Tables 4,5 and 8 ) $[20,24,61,85,88,100,118-120]$ using carbohydrates as acceptors. The most extreme A:D ratio of 1:5000 was used in a thioglycoligase reaction carried out with a mutant of the hexosaminidase from Streptomyces plicatus, which was able to S-glycosylate small peptides and proteins [24]. Such a low A:D ratio could only be achieved because of the different type of reaction.

\section{3. $\mathrm{pH}$ and Temperature Modifying Trans-Glycosylation Activity}

In contrast to other trans-glycosylases [123], there are no specific reports of increased trans-glycosylation activity for GH20s by modifying the $\mathrm{pH}$. However, two cases of independent studies using varying conditions for the same enzyme in similar reactions exist. Three independent studies investigated the auto-condensation of pNP-GlcNAc catalyzed by AoHex at different $\mathrm{pH}$ and temperature conditions $[53,54,56]$, resulting in varying regioselectivity (Table 2$)$. Whereas the reaction at neutral $\mathrm{pH}(\mathrm{pH} 7.0)$ and slightly elevated temperature $\left(50^{\circ} \mathrm{C}\right)$ led to the $\beta$-1,3-linked product only [53], the main product of reactions at slightly acidic $\mathrm{pH}(\mathrm{pH} 5.5-6.0)$ and moderate temperatures $\left(35-37^{\circ} \mathrm{C}\right)$ was the $\beta-1,4$-linked product with the $\beta-1,6$-linked dimer as side product $[54,56]$. The higher trans-glycosylation yields obtained in the study using slightly acidic $\mathrm{pH}$ [56] are probably due to the higher substrate concentration and solvent addition.

Four independent studies have reported synthesis of LNT II from pNP-GlcNAc and Lac (Figure 4) using the enzyme BbhI from Bifidobacterium bifidum (Table 5) [101-104]. Two of the studies used identical conditions for the reaction $\left(\mathrm{pH} 5.8,55^{\circ} \mathrm{C}, \mathrm{A}: \mathrm{D}=20: 1,20 \%(v / v) \mathrm{DMSO}\right)$ and reported similar yields: Both, Chen et al. [103] and Schmölzer et al. [101] thus reported a yield of approximately $45 \%$. In another study, the synthesis of LNT II by BbhI was carried out at neutral $\mathrm{pH}\left(\mathrm{pH} \mathrm{7.0)}\right.$ and $37^{\circ} \mathrm{C}$, with an A:D ratio of 4:1 and without DMSO, leading to a yield of $16 \%$ (based on HPLC analysis using an internal standard) after $0.6 \mathrm{~h} \mathrm{[102].} \mathrm{In} \mathrm{the} \mathrm{other} \mathrm{two} \mathrm{studies,} \mathrm{the} \mathrm{D746A} \mathrm{and} \mathrm{D746E} \mathrm{mutants} \mathrm{of}$ BbhI, which have been used for LNT II synthesis at $\mathrm{pH} 5.8$ and at two different temperatures of 37 and $55^{\circ} \mathrm{C}[101,104]$. For the mutant D746A, the reaction at the lower temperature $\left(37^{\circ} \mathrm{C}\right)$ resulted in a higher yield of $58 \%$ [104] compared to $40 \%$ in the other study, where the reaction has been performed at $55{ }^{\circ} \mathrm{C}$ [101]. In the case of D746E the opposite trend was observed. The reaction at $37{ }^{\circ} \mathrm{C}$ gave a slightly lower yield of $63 \%$ [104] compared to 71\% [101]. Overall, the addition of DMSO and a lower $\mathrm{pH}$ seem to favor trans-glycosylation activity for this specific reaction.

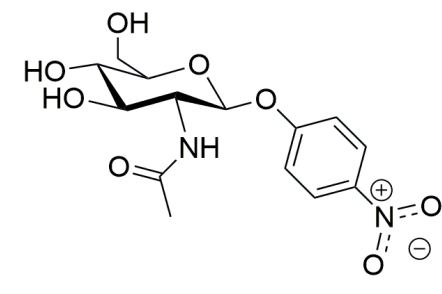

pNP-GICNAC

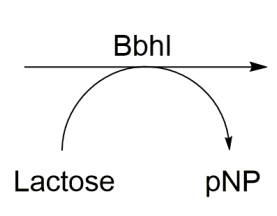

Lactose pNP

Figure 4. BbhI catalyzed trans-glycosylation reaction for synthesis of LNT II using pNP-GlcNAc as donor substrate.

\subsection{Additives Increasing Trans-Glycosylation}

Low water activity $\left(a_{\mathrm{w}}\right)$ in a solvent system favors synthetic reactions and certain enzymes are known to operate well in such non-conventional systems [124-128]. Addition of co-solvents, water-miscible solutes or salts can alter $a_{\mathrm{w}}$, leading to an increased synthetic action by hydrolases. However, whereas other hydrolytic enzymes such as proteases, esterases, or lipases can cope with even non-aqueous systems $\left(a_{\mathrm{w}}<0.01\right)$, it appears that glycosidases require at least $a_{\mathrm{w}} \geq 0.9$ to be active [129]. Nevertheless, lowering of $a_{\mathrm{w}}$ has been applied for many GHs to increase their trans-glycosylation efficiency [129-136], including GH20 hexosaminidases [21,24,46,54,56,61,62,73,87,88,90-92,95-97,100, 101,103-106,115,116,118-120]. 


\subsubsection{Co-Solvents}

In general, the most commonly added co-solvent in glycosidase-catalyzed trans-glycosylation reactions is dimethylsulfoxide (DMSO) as it is often required to dissolve the pNP-activated donor substrate. However, there are only a few studies, in which DMSO $(5 \%-20 \%(v / v))$ has been present during GH20-catalyzed trans-glycosylation (Tables 5, 9 and 10) [24,101,103-105]. Especially for the fungal enzymes it seems to be more common to add acetonitrile (MeCN, $5 \%-45 \%(v / v))$ as co-solvent to increase yields (Tables $2-4$ and Table 10) $[54,56,61,88,90-92,95-97]$. Other reported co-solvents are $\mathrm{N}, \mathrm{N}$-dimethylformamide (DMF, 5\% $(v / v))[73,106]$, dioxane $(20 \%(v / v))$ [56], 1,3-butanediol and 1,2,4-butanetriol $(8 \%(v / v))$ [109]. The most extreme case reported in literature is the use of a so-called plasticized glass phase using a total solvent content of only $20 \%$ ( $10 \%$ water, $5 \%$ ethanol, $5 \% n$-propanol) for synthesis of GlcNAc-sphingosine by AoHex $[115,116]$. As a consequence of the reaction system, very high substrate concentrations were achieved and an isolated yield of $44 \%$ was reported (Table 10 ).

\subsubsection{Salts}

Other additives to modulate $a_{\mathrm{W}}$ include inorganic, soluble salts. The concentration-dependent effect of $\left(\mathrm{NH}_{4}\right)_{2} \mathrm{SO}_{4}(93-1170 \mathrm{mM})$ on trans-glycosylation catalyzed by $\mathrm{AoHex}$ and $\mathrm{PbHex}$ (from Penicillium brasilianum) resulted in up to seven times higher yields (Tables 1, 2 and 4) [46,56,91]. Addition of $\mathrm{LiCl}(0.5 \mathrm{M})$ had similarly positive effects on yields [46]. Analogously, addition of $\mathrm{MgSO}_{4}$ $(20 \%(w / v))$ led to increased GalNAcase activity and decreased GlcNAcase activity of PoHex (from Penicillium oxalicum), which was also applied in trans-glycosylative synthesis of disaccharides [87].

\subsubsection{Cyclodextrins}

Addition of cyclodextrins is an alternative to co-solvents and salts to improve GH20 trans-glycosylation $[100,118-120]$. The addition of equimolar amounts of $\beta$-cyclodextrin to the acceptor pNP-Lac, which form an inclusion complex shielding the pNP moiety, was reported to not only lead to an increased yield by a factor of two, but also to favor formation of GlcNAc- $\beta-1,6-G a l-\beta-1,4-G l c$ over Gal- $\beta-1,4-($ GlcNAc- $\beta-1,6)$ Glc (Table 8) [119]. Similar trends were observed in follow-up studies including use of $\alpha$-cyclodextrin $[118,120]$. Later, others have directly compared the use of $\alpha-, \beta-$, and $\gamma$-hydroxypropyl- $\beta$-cyclodextrin as well as heptakis-(2,6-di-O-methyl)- $\beta$-cyclodextrin for increasing solubility of pNP-GlcNAc and pNP-GalNAc. The latter cyclodextrin had a positive effect on the kinetics for synthesis of GalNAc- $\beta-1,4-G l c N A c-\alpha-O-U D P$ (increased $V_{\max } 1.6$-fold, increased $K_{\mathrm{i}}$ 5.7-fold) [100].

\section{Increased Trans-Glycosylation Activity by Enzyme Engineering}

As an alternative to reaction engineering, trans-glycosylation yields can be increased by enzyme engineering. In contrast to the amount of available data for natural GH20 trans-glycosylases, there are only a few studies on engineering these enzymes. Nevertheless, based on the described mutations it is possible to deduce some general mutation guidelines for $\mathrm{GH} 20 \beta-\mathrm{N}$-acetylhexosaminidase engineering to increase their trans-glycosylation activity. For an overview of hexosaminidase enzyme engineering efforts to improve other protein characteristics (e.g., thermal stability) please refer to the review by Slámová and Bojarová [137].

\subsection{Mutation of the Water-Stabilizing Tyr}

The first engineering study on a GH20 enzyme was carried out on TfHex from Talaromyces flavus only five years ago [64]. In this work, the authors were inspired by a previous mutational study on a GH85 endo- $\beta-N$-acetylglucosaminidase, which revealed that mutation of the water stabilizing conserved Tyr residue in the active site to Phe led to increased trans-glycosylation activity and diminished hydrolytic activity [34]. Mutating Y470 in TfHex (Figure 5A) in a similar manner to Phe led to one of the highest yields (41\%) reported for GH20 $\beta-N$-acetylhexosaminidase catalyzed auto-condensation reactions (Table 2) [64]. Introduction of the hetero-aromatic His residue in this 
position also increased the trans-glycosylation yield, though to a somewhat lower degree (Table 2). Interestingly, both mutations also shifted the product spectrum towards longer chito-oligosaccharides $\left((\mathrm{GlcNAc})_{3}\right.$ and $\left.(\mathrm{GlcNAc})_{4}\right)$. The Y $470 \mathrm{~N}$ mutation, which was inspired by the natural presence of Asn in the same position of closely related GH84 $\beta-N$-acetylglucosaminidases, even led to synthesis of insoluble oligomers ((GlcNAc) 7 and longer, Table 2$)$. However, transferring a similar mutation (Tyr to Phe) to the bacterial enzymes BbhI and LnbB (both from Bifidobacterium bifidum) turned out to be less beneficial than in TfHex (Table 5) [101,105]. In the case of BbhI, the yield of the trans-glycosylation product was increased only to a minor extent when using the pNP-activated donor substrate due to the persistence of secondary hydrolysis. However, when using Glc-oxa as donor at least the synthesis of LNT II by BbhI-Y827F showed a 1.4-fold higher yield (80\%) compared to the wild-type (WT) (Table 9) [101]. In contrast, the LnbB-Y419F mutant showed an opposite trend: Whereas the formation of LNT from Lac and LNB-oxa by LnbB-Y419F resulted in a 45\% lower yield of the desired product compared to the WT (Table 9), the use of pNP-LNB as donor led to a 1.6-fold higher yield (Table 5) [105]. In summary, mutating the water-stabilizing Tyr to a Phe residue can be a successful strategy to increase trans-glycosylation activity in GH20 enzymes depending on the substrates and enzymes used.

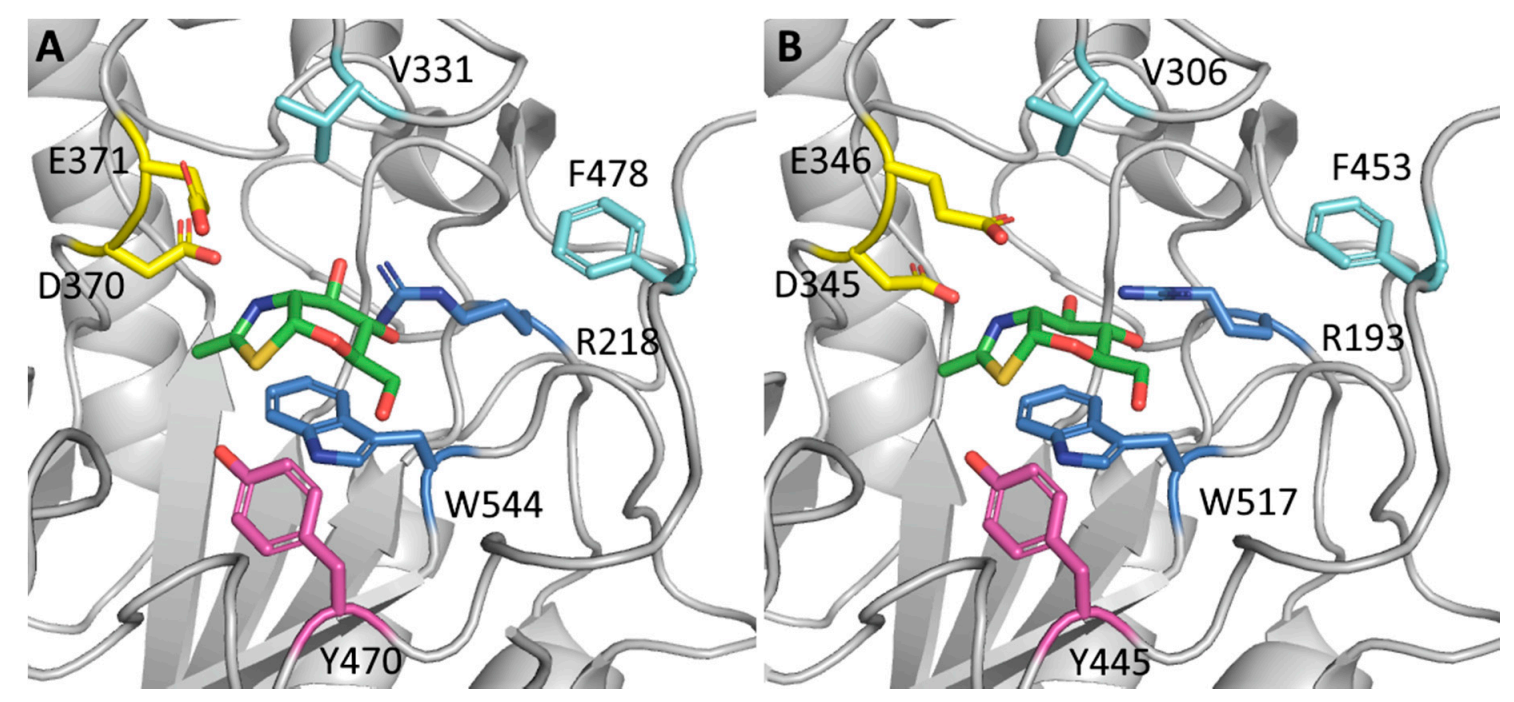

Figure 5. Active sites of two fungal $\beta-N$-acetylhexosaminidases: (A) homology model of TfHex from Talaromyces flavus with the inhibitor Glc-thiazoline docked (created with YASARA [138]); (B) crystal structure of AoHex from Aspergillus oryzae (PDB: 5OAR [139]) with the inhibitor Glc-thiazoline bound. The catalytic Asp-Glu pair is highlighted in yellow, the water-stabilizing Tyr residue is highlighted in purple, bound or docked ligands are highlighted in green, conserved residues (Arg and Trp) are highlighted in dark blue, and residues involved in aglycone binding are highlighted in light blue (Phe and Val).

\subsection{Mutation of the Aglycone Binding Site}

Another possibility to increase trans-glycosylation activity of fungal enzymes was presented at the 13th Carbohydrate Biotechnology Meeting in Toulouse, France, in 2019 by researchers from the Křen group: Mutation of the aglycone binding residues V306 and F453 in AoHex (Figure 5B) to Trp led to an increased trans-glycosylation activity and reduced hydrolytic activity [140]. Due to the close relatedness (78\% similarity) the same mutation should be possible in TfHex (Figure 5A) and other fungal $\beta$ - $N$-hexosaminidases. However, it seems that this strategy is not applicable to bacterial GH20 enzymes since these have a slightly different aglycone binding site topology, as discussed below.

\subsection{Mutation of the Catalytic Asp-Glu Pair}

A general and popular strategy to create so called glycosynthases was introduced by Stephen Withers and colleagues over 20 years ago. They demonstrated that a crippled CAZyme, in which 
the catalytic nucleophile was mutated to a non-functional Ala residue, is still able to catalyze trans-glycosylation when using a properly activated donor molecule [141]. Due to their relatively simple way of preparation, the glycosyl fluorides became popular as donor molecules for this approach. However, the fluoride approach is not applicable in industrial applications, and was moreover never followed for GH20 enzymes because of the significantly different reaction mechanism. While Glc-oxa should in theory be a properly activated donor molecule, its low stability at low $\mathrm{pH}$ hinders the creation of GH20 glycosynthases from fungal $\beta-N$-acetylhexosaminidases since these require a low $\mathrm{pH}$ for optimal activity (Tables 3 and 4). The discovery of bacterial GH20 enzymes with pH optimum $\geq 7.0$ (e.g., Hex1, Table 8) [109] paved the way for the glycosynthase approach in GH20 by mutating the catalytic Asp. The power of such a GH20 glycosynthase for LNT II synthesis (Figure 6) from Lac and Glc-oxa (A:D $=1: 1$, concentration of both $=600 \mathrm{mM}$ ) was recently convincingly demonstrated using the BbhI-D746E mutant, which resulted in $86 \%$ isolated yield $(281 \mathrm{~g} / \mathrm{L})$ in a $30 \mathrm{~min}$ reaction at $\mathrm{pH} 7.5$ (Table 9) [101]. This result was a 1.5-fold yield increase compared to the WT (58\%; Table 9). Alternative mutations of the same residue (D746A and D746Q) led to real glycosynthases with completely abolished hydrolytic activity, but also to significantly slower enzymes, which was reflected in the lower yield after a longer reaction time (Table 9). Surprisingly, transfer of these mutations to LnbB was not as successful. Both mutants (D320E and D320A, Table 9) were significantly slower in synthesis of LNT from Lac and LNB-oxa (Figure 6) leading to less than 50\% of the wild-type yield in more than ten times longer reactions [105]. Finally, a similar 1.5-fold increase in yield compared to the WT was obtained with BbhI-D746E using a pNP-donor molecule, but the obtained yield was much lower (18\%; Table 5) [101]. Recently, a new mutational study on BbhI combining directed evolution and site saturation mutagenesis (SSM) showed that the BbhI-D746T led to an almost doubled yield of LNT II when using Lac and pNP-GlcNAc as substrates. The maximum reported yield was 85\% (Table 5) [104]. Furthermore, we can conclude from the SSM of position D746 that introduction of any residue bigger than Glu lead to complete inactivation of the enzyme, probably due to steric hindrance of substrate binding, (Table 5). Similarly, introduction of the two amino acid amides Asn and Gln in this position also lead to enzyme inactivation, although varying results were obtained in two independent studies on BbhI-D746Q (Table 5).

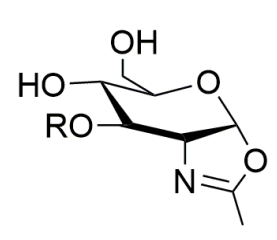

Glc-oxa: $\mathrm{R}=\mathrm{H}$

LNB-oxa: $\mathrm{R}=\mathrm{Gal}$

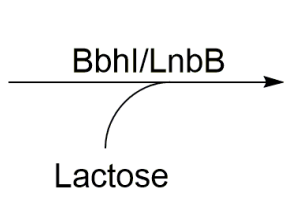

Lactose

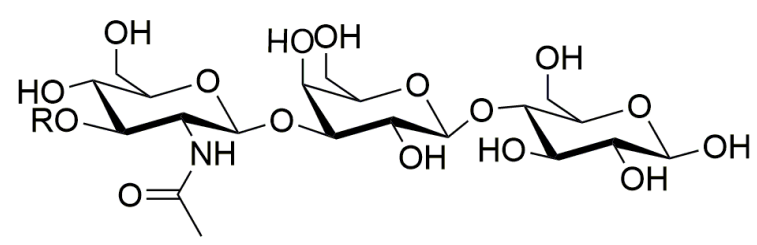

LNT II: $\mathrm{R}=\mathrm{H}$

LNT: $\mathrm{R}=\mathrm{Gal}$

Figure 6. BbhI and LnbB catalyzed trans-glycosylation reaction for synthesis of LNT II and LNT, respectively, using Glc-oxa as donor substrate.

Mutation of the catalytic Glu residue in $S p H e x$ to a non-functional Ala as recently described by Tegl et al. enabled a thioglycoligase reaction (Tables 5, 9 and 10) [24]. Normally, the catalytic Glu residue is required for protonation of the glycosidic bond, which in turn leads to release of the leaving group. Thus, a mutation in this position would be expected to lead to enzyme inactivation. However, the Withers group demonstrated almost 20 years ago that the activity of such a mutated GH can actually be rescued by the use of nucleophiles with a low $\mathrm{p} K_{\mathrm{a}}$ value such as thiols, which leads to the formation of thioglycosides [142]. The SpHex-E314A mutant was not only able to catalyze synthesis of thioglycosides from pNP-GlcNAc and Glc-oxa as donor substrates and the respective thio-sugars as acceptors, but could also catalyze the transfer of a GlcNAc moiety to the free amino acid Cys as well as to Cys-containing peptides and proteins leading to GlcNAc-peptide/protein conjugates [24]. However, such a mutant is not expected to synthesize conventional $O$-glycosidic bonds due to the rather high 
$\mathrm{p} K_{\mathrm{a}}$ values found in carbohydrates. Product formation of $O$-glycosides was only observed when using the low $\mathrm{p} K_{\mathrm{a}}$ nucleophiles $\mathrm{pNP}$ and 2,4-dinitrophenol [24].

\subsection{Mutation of Other Conserved Active Site Residues}

Another generic approach to increase trans-glycosylation activity of CAZymes is the mutation of other conserved residues in the active site to structurally related residues (e.g., from Tyr to Phe), which was first described in 2014 for a GH1 $\beta$-glycosidase [143] and has been transferred to many other GH families since then [144-146]. Recently, six conserved positions were identified from an alignment of $585 \mathrm{GH} 20$ sequences and their effect on the trans-glycosylation activity of BbhI was studied [102]. The two mutants R577K and W288H (Figure 7A) led to a doubled and quadrupled yield of LNT II, respectively, in reactions with Lac and pNP-GlcNAc as substrates (Table 5) [102]. These residues are present in both fungal and bacterial GH20 $\beta$-N-acetylhexosaminidases (residues highlighted in dark blue in Figures 5 and 7). Thus, the corresponding residues could be targeted in other GH20s exhibiting some natural trans-glycosylation activity, as the latter is a requirement for this engineering strategy [102].

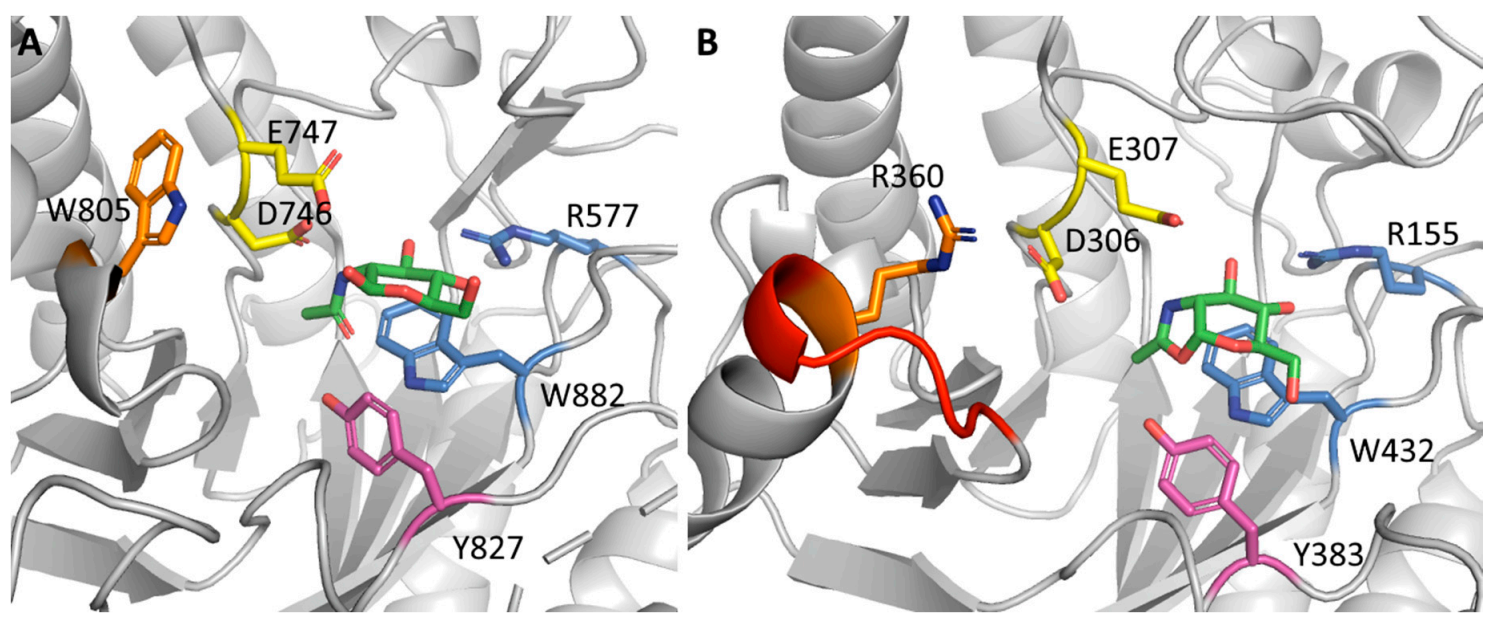

Figure 7. Active sites models of two bacterial $\beta-N$-acetylhexosaminidases: (A) homology model of BbhI from Bifidobacterium bifidum (created with YASARA [138]) with the mutated W805 [104] highlighted in orange and GlcNAc bound in the active site; (B) homology model of Hex1GTEPG [117] with the inserted loop highlighted in red and the newly positioned R360 in orange; the reaction intermediate Glc-oxa was docked into the structure [117]. The catalytic Asp-Glu pair is highlighted in yellow, the water-stabilizing Tyr residue is highlighted in purple, bound or docked ligands are highlighted in green, and conserved residues (Arg and Trp) are highlighted in dark blue.

\subsection{A Non-Conserved Loop Close to the Active Site as Hotspot for Beneficial Mutation?}

Lastly, we would like to highlight a potential hotspot for mutations that drive trans-glycosylation activity in GH20 enzymes. We previously demonstrated that introduction of a specific loop, which was identified in related GH20 sequences from pathogens, into Hex1 led to a $>5$-fold increased trans-glycosylation product yield in reactions with Lac as acceptor and (GlcNAc) $)_{2}$ as donor (Figure 7B, Table 8) [117]. Additionally, in a recent mutational study on BbhI, the mutant W805R (Figure 7A) was identified as the one with superior trans-glycosylation activity [104].

Interestingly, when comparing the homology models of BbhI and the Hex1-GTEPG loop mutant (Figure 7), we noticed that the mutated W805 in BbhI and the newly positioned R360 in Hex1-GTEPG are in a similar position with respect to the active site. Therefore, we dare to speculate that an Arg residue in this position is beneficial for trans-glycosylation reactions using GH20 $\beta$-N-acetylhexosaminidases. Such an Arg residue might be responsible for modulating the water network or binding water in general, which leads to lowered availability of water for hydrolysis, as proposed for GH33 trans-sialidases [147]. 
However, in case of Hex1 the inserted loop sequence itself also seems to have an important role with respect to trans-glycosylation activity, since not all alternative loop sequences of the same length were as beneficial as others [117]. Only the additional loops carrying a negative charge in the middle of the five amino acid sequence (GTEPG and GTDDA) led to an increased trans-glycosylation yield. Other tested loops carrying a positive charge (SFRTP) or a negative charge in a different position (DFVTP) led to a decreased trans-glycosylation activity [117].

\section{Conclusions}

GH20 $\beta$ - $N$-acetylhexosaminidases are gaining significant attention for production of functional molecules, especially biomimetic human milk oligosaccharides, via their ability to catalyze enzymatic transfer of GlcNAc- and to a lesser extent GalNAc- via reverse hydrolysis or trans-glycosylation. Careful assessment of the available literature data showed that trans-glycosylation is more favorable than reverse hydrolysis with regard to efficiency and product yields. Indeed, a significant body of data have been reported on trans-glycosylation reactions promoted by microbially derived GH20 $\beta-N$-acetylhexosaminidases. Notably, a large amount of data have been reported for reactions catalyzed by the AoHex enzyme and the many different mutants of this enzyme derived from Aspergillus oryzae, and more recently also for other GH20 enzymes, in particular from Bifidobacterium bifidum. Surprisingly, it is not possible to discern any clear trends with respect to $\mathrm{pH}$ and temperature on GH20 trans-glycosylation reactions, but reaction engineering involving various types of donor activation, high A:D ratio, high substrate concentration in general, lowering of $a_{w}$ by addition of co-solvents, salts or addition of cyclodextrins can lead to increased yields or altered regioselectivity. The distinct catalytic mechanism of the GH20 $\beta-N$-acetylhexosaminidases, involving substrate-assisted catalysis in which the 2-acetamido group acts as an intramolecular nucleophile leading to formation of an oxazolinium ion intermediate, has proven uniquely useful as a blueprint for using oxazoline-conjugated substrates for trans-glycosylation. Although chitin, e.g., from shrimp or crab waste streams, may seem an obvious substrate for sustainable $\beta-N$-acetylhexosaminidase catalyzed trans-glycosylation processes, we anticipate that oxazoline-conjugated donor substrates have more potential for industrial development of these reactions. Insight into the details of the active site topology and the function of the different amino acids in both the fungal and bacterial GH20 enzymes indicate that trans-glycosylation activity may be promoted by the following protein engineering steps: Mutation of the aglycone binding site to large hydrophobic residues, mutation of the water-stabilizing Tyr as well as other conserved residues, notably Trp and Arg. Efficient glycosynthases using oxazoline substrates can be created by mutation of the catalytic Asp. Recent work suggested that introduction or repositioning of an Arg residue near the active site, e.g., by loop engineering, may be a hotspot for creating beneficial mutations for trans-glycosylation. Based on this foundation, we anticipate the development of novel protein engineering and reaction optimization strategies allowing further technological advances to promote exploration of GH20 enzymes for synthesis of distinct functional carbohydrates and glycan conjugates.

Author Contributions: Conceptualization, J.M., A.S.M., and B.Z.; literature search, J.M.; writing and original draft preparation, J.M.; writing and editing, J.M., M.V., A.S.M., B.Z.; visualization, J.M.; supervision, A.S.M. and B.Z.; project administration, B.Z.; funding acquisition, B.Z. and A.S.M. All authors have read and agree to the published version of the manuscript.

Funding: This work was supported by The Technical University of Denmark; The Innovation Fund Denmark Grand Solutions Grant no. 9068-00006B; and the Novo Nordisk Foundation Grant no. NNF-17OC0027742.

Conflicts of Interest: The authors declare no conflicts of interest.

\section{Abbreviations}

For amino acids and nucleotides the standard one and three letter abbreviations were used. For other non-standard abbreviations the reader is referred to the following list:Ac-acetyl, CAZy-Carbohydrate active enzyme, DMF-N,N-dimethylformamide, DMSO—dimethylsulfoxide, Doo-triethylene glycol, Et—ethyl, $\mathrm{EtN}_{3}$-azidoethyl, EtOH - ethanol, Gal-galactose, GalNAc- $\beta-N$-acetylgalactosamine, GH-Glycoside hydrolase, Glc-glucose, GlcANAc- $\beta-N$-acetylglucurosaminic acid, GlcNAc- $\beta-N$-acetylglucosamine, 
GlcNFo- $\beta-N$-formylglucosamine, GlcNGl- $\beta-N$-glycolylglucosamine, GlcNPr- $\beta$ - $N$-propionylglucosamine, GT-glycosyltransferease, Hx-1,6-hexanediol, Lac-lactose, LacNAc-Gal- $\beta$-1,4-GlcNAc, LNB-Lacto- $N$-biose (Gal- $\beta$-1,3-GlcNAc), LNT-Lacto- $N$-tetraose (Gal- $\beta-1,3-G l c N A c-\beta-1,3-G a l-\beta-1,4-G l c)$, LNnT-Lacto- $N$-neotetraose (Gal- $\beta$-1,4-GlcNAc- $\beta-1,3-G a l-\beta-1,4-G l c)$, LNT II-lacto- $N$-triose II (GlcNAc $\beta$-1,3-Gal- $\beta$-1,4-Glc), Man-mannose, ManNAc- $\beta$ - $N$-acetylmannosamine, Me-methyl, MeCN-acetonitrile, $\mathrm{N}_{3}$-azido, NPy-3-nitro-2-pyridyl, oNP—ortho-nitro phenyl, oxa-oxazoline, $\mathrm{Ph}$ - phenyl, pNP—para-nitro phenyl, $\mathrm{Pr}$ - propyl, $\mathrm{PrOH}$ - propanol, $\mathrm{SSM}$ - site saturation mutagenesis, $t$-Boc—tert-butyloxycarbonyl, $\mathrm{SO}_{3}$ - sulfo.

\section{References}

1. Hackman, R.H.; Goldberg, M. Light-scattering and infrared-spectrophotometric studies of chitin and chitin derivatives. Carbohydr. Res. 1974, 38, 35-45. [CrossRef]

2. Gooday, G. The Ecology of Chitin Degradation. In Advances in Microbial Ecology; Marshall, K., Ed.; Springer: Boston, MA, USA, 1990; pp. 387-430.

3. Slámová, K.; Bojarová, P.; Petrásková, L.; Křen, V. $\beta-N$-Acetylhexosaminidase: What's in a name...? Biotechnol. Adv. 2010, 28, 682-693. [CrossRef] [PubMed]

4. Liu, T.; Duan, Y.; Yang, Q. Revisiting glycoside hydrolase family $20 \beta$-N-acetyl-D-hexosaminidases: Crystal structures, physiological substrates and specific inhibitors. Biotechnol. Adv. 2018, 36, 1127-1138. [CrossRef] [PubMed]

5. Wendland, J.; Schaub, Y.; Walther, A. N-acetylglucosamine utilization by Saccharomyces cerevisiae based on expression of Candida albicans NAG genes. Appl. Environ. Microbiol. 2009, 75, 5840-5845. [CrossRef] [PubMed]

6. Chen, J.K.; Shen, C.R.; Liu, C.L. N-acetylglucosamine: Production and applications. Mar. Drugs 2010, 8, 2493-2516. [CrossRef] [PubMed]

7. Faijes, M.; Castejón-Vilatersana, M.; Val-Cid, C.; Planas, A. Enzymatic and cell factory approaches to the production of human milk oligosaccharides. Biotechnol. Adv. 2019, 37, 667-697. [CrossRef]

8. Zeuner, B.; Teze, D.; Muschiol, J.; Meyer, A.S. Synthesis of Human Milk Oligosaccharides: Protein Engineering Strategies for Improved Enzymatic Transglycosylation. Molecules 2019, 24, 2033. [CrossRef]

9. Bode, L. Human milk oligosaccharides: Every baby needs a sugar mama. Glycobiology 2012, 22, 1147-1162. [CrossRef]

10. Bode, L. The functional biology of human milk oligosaccharides. Early Hum. Dev. 2015, 91, 619-622. [CrossRef]

11. Bych, K.; Mikš, M.H.; Johanson, T.; Hederos, M.J.; Vigsnæs, L.K.; Becker, P. Production of HMOs using microbial hosts-From cell engineering to large scale production. Curr. Opin. Biotechnol. 2019, 56, 130-137. [CrossRef]

12. Frahn, J.; Edgar, J.; Jones, A.; Cockrum, P.; Anderton, N.; Culvenor, C. Structure of the corynetoxins, metabolites of Corynebacterium rathayi responsible for toxicity of annual ryegrass (Lolium rigidum) pastures. Aust. J. Chem. 1984, 37, 165-182. [CrossRef]

13. Wright, D. The Orthosomycins, A New Family of Antibiotics. Tetrahedron 1979, 35, 1207-1237. [CrossRef]

14. Ngo, D.-N.; Kim, M.-M.; Kim, S.-K. Chitin oligosaccharides inhibit oxidative stress in live cells. Carbohydr. Polym. 2008, 74, 228-234. [CrossRef]

15. Ngo, D.-N.; Lee, S.-H.; Kim, M.-M.; Kim, S.-K. Production of chitin oligosaccharides with different molecular weights and their antioxidant effect in RAW 264.7 cells. J. Funct. Foods 2009, 1, 188-198. [CrossRef]

16. Wang, Z.; Zheng, L.; Yang, S.; Niu, R.; Chu, E.; Lin, X. N-Acetylchitooligosaccharide is a potent angiogenic inhibitor both in vivo and in vitro. Biochem. Biophys. Res. Commun. 2007, 357, 26-31. [CrossRef]

17. Latxague, L.; Gaubert, A.; Barthélémy, P. Recent Advances in the Chemistry of Glycoconjugate Amphiphiles. Molecules 2018, 23, 89. [CrossRef]

18. Micheletto, Y.M.S.; da Silveira, N.P.; Barboza, D.M.; dos Santos, M.C.; de Lima, V.R.; Giacomelli, F.C.; Martinez, J.C.V.; Frizon, T.E.A.; Bó, A.G.D. Investigation of self-association between new glycosurfactant $\mathrm{N}$-acetyl- $\beta$-D-glucosaminyl-PEG-docosanate and soybean phosphatidylcholine into vesicles. Colloids Surf. A Physicochem. Eng. Asp. 2015, 467, 166-172. [CrossRef]

19. Kieburg, C.; Lindhorst, T.K.; Křen, V. Enzymatic Glycosylation of Branched Symmetrical Non-Carbohydrate Polyols. J. Carbohydr. Chem. 1998, 17, 1239-1247. [CrossRef] 
20. Misawa, Y.; Akimoto, T.; Amarume, S.; Murata, T.; Usui, T. Enzymatic synthesis of spacer-linked divalent glycosides carrying $\mathrm{N}$-acetylglucosamine and $\mathrm{N}$-acetyllactosamine: Analysis of cross-linking activities with WGA. J. Biochem. 2008, 143, 21-30. [CrossRef]

21. Křen, V.; Ščigelová, M.; Přikrylová, V.; Havliček, V.; Sedmera, P. Enzymatic Synthesis of $\beta$-N-Acetylhexosaminides of Ergot Alkaloids. Biocatalysis 1994, 10, 181-193. [CrossRef]

22. Křen, V.; Huňkova, Z.; Halada, P.; Suzuki, Y. Transglycosylation of thiamin by fungal $\beta-N$-acetylhexosaminidases. Biosci. Biotechnol. Biochem. 1998, 62, 2415-2417. [CrossRef] [PubMed]

23. Weignerová, L.; Pelantová, H.; Manglová, D.; Michálková, K.; Křen, V. Condensation reactions catalyzed by $\alpha-N$-acetylgalactosaminidase from Aspergillus niger yielding $\alpha$ - $N$-acetylgalactosaminides. Biocatal. Biotransform. 2010, 28, 150-155. [CrossRef]

24. Tegl, G.; Hanson, J.; Chen, H.M.; Kwan, D.H.; Santana, A.G.; Withers, S.G. Facile Formation of $\beta$-thioGlcNAc Linkages to Thiol-Containing Sugars, Peptides, and Proteins using a Mutant GH20 Hexosaminidase. Angew. Chem. Int. Ed. Engl. 2019, 58, 1632-1637. [CrossRef] [PubMed]

25. Lombard, V.; Golaconda Ramulu, H.; Drula, E.; Coutinho, P.M.; Henrissat, B. The carbohydrate-active enzymes database (CAZy) in 2013. Nucleic Acids Res. 2014, 42, D490-D495. [CrossRef]

26. Drouillard, S.; Armand, S.; Davies, G.J.; Vorgias, C.E.; Henrissat, B. Serratia marcescens chitobiase is a retaining glycosidase utilizing substrate Acetamido Group Participation. Biochem. J. 1997, 949, 945-949. [CrossRef]

27. Mega, T.; Ikenaka, T.; Matsushima, Y. Studies on N-Acetyl- $\beta$-D-glucosaminidase of Aspergillus oryzae I. Purification and Characterization of N-Acetyl- $\beta$-D-glucosaminidase. J. Biochem. 1970, 68, $109-117$.

28. Jones, C.S.; Kosman, D.J. Purification, Properties, Kinetics, and Mechanism of $\beta$-N-Acetylglucosaminidase from Aspergillus niger. J. Biol. Chem. 1980, 255, 11861-11869.

29. Knapp, S.; Vocadlo, D.; Gao, Z.; Kirk, B.; Lou, J.; Withers, S.G. NAG-thiazoline, An N-Acetyl- $\beta$-hexosaminidase Inhibitor That Implicates Acetamido Participation. J. Am. Chem. Soc. 1996, 118, 6804-6805. [CrossRef]

30. Tews, I.; Perrakis, A.; Oppenheim, A.; Dauter, Z.; Wilson, K.S.; Vorgias, C.E. Bacterial chitobiase structure provides insight into catalytic mechanism and the basis of Tay-Sachs disease. Nat. Struct. Biol. 1996, 3, 638-648. [CrossRef]

31. Koshland, D.E., Jr. Stereochemistry and the Mechanism of Enzymatic Reactions. Biol. Rev. 1953, $28,416-436$. [CrossRef]

32. Teze, D.; Coines, J.; Raich, L.; Kalichuk, V.; Solleux, C.; Tellier, C.; André-Miral, C.; Svensson, B.; Rovira, C. A single point mutation converts GH84 O-GlcNAc hydrolases into phosphorylases. Experimental and theoretical evidence. J. Am. Chem. Soc. 2020, 142, 2120-2124. [CrossRef] [PubMed]

33. Coines, J.; Alfonso-Prieto, M.; Biarnés, X.; Planas, A.; Rovira, C. Oxazoline or Oxazolinium Ion? The Protonation State and Conformation of the Reaction Intermediate of Chitinase Enzymes Revisited. Chem. A Eur. J. 2018, 24, 19258-19265. [CrossRef] [PubMed]

34. Umekawa, M.; Huang, W.; Li, B.; Fujita, K.; Ashida, H.; Wang, L.-X.; Yamamoto, K. Mutants of Mucor hiemalis Endo- $\beta$ - $N$-acetylglucosaminidase Show Enhanced Transglycosylation and Glycosynthase-like Activities. J. Biol. Chem. 2008, 283, 4469-4479. [CrossRef] [PubMed]

35. Bissaro, B.; Monsan, P.; Fauré, R.; O’Donohue, M.J. Glycosynthesis in a waterworld: New insight into the molecular basis of transglycosylation in retaining glycoside hydrolases. Biochem. J. 2015, 467, 17-35. [CrossRef]

36. Bojarová, P.; Křen, V. Glycosidases: A key to tailored carbohydrates. Trends Biotechnol. 2009, 27, $199-209$. [CrossRef]

37. Bojarovà, P.; Křen, V. Glycosidases in carbohydrate synthesis: When organic chemistry falls short. CHIMIA Int. J. Chem. 2011, 65, 65-70. [CrossRef]

38. Bojarová, P.; Bruthans, J.; Křen, V. $\beta$-N-Acetylhexosaminidases-The wizards of glycosylation. Appl. Microbiol. Biotechnol. 2019, 103, 7869-7881. [CrossRef]

39. Vocadlo, D.J.; Withers, S.G. Detailed Comparative Analysis of the Catalytic Mechanisms of $\beta$-N-Acetylglucosaminidases from Families 3 and 20 of Glycoside Hydrolases. Biochemistry 2005, 44, 12809-12818. [CrossRef]

40. Sakurama, H.; Kiyohara, M.; Wada, J.; Honda, Y.; Yamaguchi, M.; Fukiya, S.; Yokota, A.; Ashida, H.; Kumagai, H.; Kitaoka, M.; et al. Lacto-N-biosidase Encoded by a Novel Gene of Bifidobacterium longum Subspecies longum Shows Unique Substrate Specificity and Requires a Designated Chaperone for Its Active Expression. J. Biol. Chem. 2013, 288, 25194-25206. [CrossRef] 
41. Biosynth Carbosynth, UDP-GlcNAc Disodium Salt. Available online: https://www.carbosynth. com/carbosynth/website.nsf/(w-productdisplay)/218264098DDF79EC802573EE0073054C (accessed on 31 January 2020).

42. Zeuner, B.; Jers, C.; Mikkelsen, J.D.; Meyer, A.S. Methods for improving enzymatic trans-glycosylation for synthesis of human milk oligosaccharide biomimetics. J. Agric. Food Chem. 2014, 62, 9615-9631. [CrossRef]

43. Kasche, V. Mechanism and yields in enzyme catalysed equilibrium and kinetically controlled synthesis of $\beta$-lactam antibiotics, peptides and other condensation products. Enzym. Microb. Technol. 1986, 8, 4-16. [CrossRef]

44. Ajisaka, K.; Nishida, H.; Fujimoto, H. The synthesis of oligosaccharides by the reversed hydrolysis reaction of $\beta$-glucosidase at high substrate concentration and at high temperature. Biotechnol. Lett. 1987, 9, 243-248. [CrossRef]

45. Fujimoto, H.; Isomura, M.; Miyazaki, T.; Matsuo, I.; Walton, R.; Sakakibara, T.; Ajisaka, K. Enzymatic syntheses of GlcNAc $\beta 1-2 \mathrm{Man}$ and Gal $\beta 1-4 \mathrm{GlcNAc} \beta 1-2 \mathrm{Man}$ as components of complex type sugar chains. Glycoconj. J. 1997, 14, 75-80. [CrossRef] [PubMed]

46. Rajnochová, E.; Dvořáková, J.; Huňková, Z.; Křen, V. Reverse hydrolysis catalysed by $\beta-N$-acetylhexosaminidase from Aspergillus oryzae. Biotechnol. Lett. 1997, 19, 869-872. [CrossRef]

47. Zhuravleva, N.V.; Luk'yanov, P.A.; Pivkin, M.V. N-Acetyl- $\beta$-D-hexosaminidase Secreted by the Marine Fungus Phoma glomerata. Appl. Biochem. Microbiol. 2004, 40, 448-453. [CrossRef]

48. Mega, T.; Ikenaka, T.; Matsushima, Y. Studies on N-Acetyl- $\beta$-D-glucosaminidase of Aspergillus oryzae III. Transglycosylation by the Enzyme and Preparation of Crystalline Phenyl $N, N^{\prime}$-Diacetyl- $\beta$-chitobioside. J. Biochem. 1972, 72, 1197-1203. [CrossRef]

49. Lakshmanan, T.; Loganathan, D. Enzymatic synthesis of $N$-glycoprotein linkage region disaccharide mimetics using $\beta$-N-acetylhexosaminidases from Aspergillus oryzae and Vigna radiata. Tetrahedron Asymmetry 2005, 16, 255-260. [CrossRef]

50. Matsuo, I.; Kim, S.; Yamamoto, Y.; Ajisaka, K.; Maruyama, J.I.; Nakajima, H.; Kitamoto, K. Cloning and overexpression of $\beta-N$-acetylglucosaminidase encoding gene nagA from Aspergillus oryzae and enzyme-catalyzed synthesis of human milk oligosaccharide. Biosci. Biotechnol. Biochem. 2003, 67, 646-650. [CrossRef]

51. Wada, J.; Ando, T.; Kiyohara, M.; Ashida, H.; Kitaoka, M.; Yamaguchi, M.; Kumagai, H.; Katayama, T.; Yamamoto, K. Bifidobacterium bifidum lacto-N-biosidase, a critical enzyme for the degradation of human milk oligosaccharides with a type 1 structure. Appl. Environ. Microbiol. 2008, 74, 3996-4004. [CrossRef]

52. Rauvolfová, J.; Weignerová, L.; Kuzma, M.; Přikrylová, V.; Macková, M.; Pišvejcová, A.; Křen, V. Enzymatic synthesis of $\mathrm{N}$-acetylglucosaminobioses by reverse hydrolysis: Characterisation and application of the library of fungal $\beta$-N-acetylhexosaminidases. J. Mol. Catal. B Enzym. 2004, 29, 259-264. [CrossRef]

53. Nilsson, K.G.I.; Eliasson, A.; Pan, H.; Rohman, M. Synthesis of disaccharide derivatives employing $\beta$-N-acetyl-D-hexosaminidase, $\beta$-D-galactosidase and $\beta$-D-glucuronidase. Biotechnol. Lett. 1999, 21, 11-15. [CrossRef]

54. Zeng, X.; Sun, Y.; Ye, H.; Liu, J.; Uzawa, H. Synthesis of p-nitrophenyl sulfated disaccharides with $\beta$-D-(6-sulfo)-GlcNAc units using $\beta$-N-acetylhexosaminidase from Aspergillus oryzae in a transglycosylation reaction. Biotechnol. Lett. 2007, 29, 1105-1110. [CrossRef] [PubMed]

55. Singh, S.; Packwood, J.; Samuel, C.J.; Critchley, P.; Crout, D.H.G. Glycosidase-catalysed oligosaccharide synthesis: Preparation of $N$-acetylchitooligosaccharides using the $\beta$ - $N$-acetylhexosaminidase of Aspergillus oryzae. Carbohydr. Res. 1995, 279, 293-305. [CrossRef]

56. Kubisch, J.; Weignerová, L.; Kötter, S.; Lindhorst, T.K.; Sedmera, P.; Křen, V. Enzymatic synthesis of p-nitrophenyl $\beta$-chitobioside. J. Carbohydr. Chem. 1999, 18, 975-984. [CrossRef]

57. Singh, S.; Gallagher, R.; Derrick, P.J.; Crout, D.H.G. Glycosidase-catalysed oligosaccharide synthesis: Preparation of the $N$-acetylchitooligosaccharides penta- $N$-acetylchitopentaose and hexa- $N$-acetylchitohexaose using the $\beta-N$-acetylhexosaminidase of Aspergillus oryzae. Tetrahedron Asymmetry 1995, 6, 2803-2810. [CrossRef]

58. Dvořáková, J.; Schmidt, D.; Huňková, Z.; Thiem, J.; Křen, V. Enzymatic rearrangement of chitine hydrolysates with $\beta$-N-acetylhexosaminidase from Aspergillus oryzae. J. Mol. Catal. B Enzym. 2001, 11, 225-232. [CrossRef] 
59. Tsujibo, H.; Kondo, N.; Tanaka, K.; Miyamoto, K.; Baba, N.; Inamori, Y. Molecular Analysis of the Gene Encoding a Novel Transglycosylative Enzyme from Alteromonas sp. Strain O-7 and Its Physiological Role in the Chitinolytic System. J. Bacteriol. 1999, 181, 5461-5466. [CrossRef]

60. Nanjo, F.; Ishikawa, M.; Katsumi, R.; Sakai, K. Purification, Properties, and Transglycosylation Reaction of $\beta$-N-Acetylhexosaminidase from Nocardia orientalis. Agric. Biol. Chem. 1990, 54, 899-906. [CrossRef]

61. Fialová, P.; Weignerová, L.; Rauvolfová, J.; Přikrylová, V.; Pišvejcová, A.; Ettrich, R.; Kuzma, M.; Sedmera, P.; Křen, V. Hydrolytic and transglycosylation reactions of $N$-acyl modified substrates catalysed by $\beta-N$-acetylhexosaminidases. Tetrahedron 2004, 60, 693-701. [CrossRef]

62. Kurakake, M.; Goto, T.; Ashiki, K.; Suenaga, Y.; Komaki, T. Synthesis of new glycosides by transglycosylation of $\mathrm{N}$-acetylhexosaminidase from Serratia marcescens YS-1. J. Agric. Food Chem. 2003, 51, 1701-1705. [CrossRef]

63. Bojarová, P.; Slámová, K.; Křenek, K.; Gažák, R.; Kulik, N.; Ettrich, R.; Pelantová, H.; Kuzma, M.; Riva, S.; Adámek, D.; et al. Charged hexosaminides as new substrates for $\beta$ - $N$-acetylhexosaminidase-catalyzed synthesis of immunomodulatory disaccharides. Adv. Synth. Catal. 2011, 353, 2409-2420. [CrossRef]

64. Slámová, K.; Krejzová, J.; Marhol, P.; Kalachova, L.; Kulik, N.; Pelantová, H.; Cvačka, J.; Křen, V. Synthesis of Derivatized Chitooligomers using Transglycosidases Engineered from the Fungal GH20 $\beta$-N-Acetylhexosaminidase. Adv. Synth. Catal. 2015, 357, 1941-1950. [CrossRef]

65. Slámová, K.; Gažák, R.; Bojarová, P.; Kulik, N.; Ettrich, R.; Pelantová, H.; Sedmera, P.; Křen, V. 4-deoxy-substrates for $\beta-N$-acetylhexosaminidases: How to make use of their loose specificity. Glycobiology 2010, 20, 1002-1009. [CrossRef] [PubMed]

66. Fialová, P.; Carmona, A.T.; Robina, I.; Ettrich, R.; Sedmera, P.; Přikrylová, V.; Petrásková-Hušáková, L.; Křen, V. Glycosyl azide-A novel substrate for enzymatic transglycosylations. Tetrahedron Lett. 2005, 46, 8715-8718. [CrossRef]

67. Takahashi, M.; Mashiyama, T.; Suzuki, T. Purification and some characteristics of $\beta$ - $N$-acetylglucosaminidase Produced by Vibrio sp. J. Ferment. Bioeng. 1993, 76, 356-360. [CrossRef]

68. Biosynth Carbosynth, 4-Nitrophenyl 2-Acetamido-2-Deoxy- $\beta$-D-Glucopyranoside. Available online: https:// www.carbosynth.com/carbosynth/website.nsf/(w-productdisplay)/1FFC21817D596F5C802570E5006E19C1 (accessed on 31 January 2020).

69. Murata, T.; Inukai, T.; Suzuki, M.; Yamagishi, M.; Usui, T. Facile enzymatic conversion of lactose into lacto- $N$-tetraose and lacto-N-neotetraose. Glycoconj. J. 1999, 16, 189-195. [CrossRef]

70. National Center for Biotechnology Information (PubChem Database), 4-Nitrophenol (CID = 980). Available online: https://pubchem.ncbi.nlm.nih.gov/compound/980 (accessed on 31 January 2020).

71. Mega, T.; Ikenaka, T.; Matsushima, Y. Studies on N-Acetyl- $\beta$-D-glucosaminidase of Aspergillus oryzae IV. Acceptor Specificity and Quantitative Representation of the Transglycosylation Reaction. J. Biochem. 1972, 72, 1391-1396. [CrossRef]

72. Yasukochi, T.; Inaba, C.; Fukase, K.; Kusumoto, S. Nitropyridyl glycosides: New glycosyl donors for enzymatic transglycosylation. Tetrahedron Lett. 1999, 40, 6585-6589. [CrossRef]

73. Nilsson, K.G.I. Enzymic synthesis of HexNAc-containing disaccharide glycosides. Carbohydr. Res. 1990, 204, 79-83. [CrossRef]

74. National Center for Biotechnology Information (PubChem Database), 2-Hydroxy-3-Nitropyridine (CID = 22793). Available online: https://pubchem.ncbi.nlm.nih.gov/compound/2-Hydroxy-3-nitropyridine (accessed on 31 January 2020).

75. National Center for Biotechnology Information (PubChem Database), 2-Nitrophenol (CID = 6947). Available online: https://pubchem.ncbi.nlm.nih.gov/compound/2-Nitrophenol (accessed on 31 January 2020).

76. National Center for Biotechnology Information (PubChem Database), Phenol (CID = 996). Available online: https://pubchem.ncbi.nlm.nih.gov/compound/Phenol (accessed on 31 January 2020).

77. National Center for Biotechnology Information (PubChem Database), Sodium Azide (CID = 33557). Available online: https://pubchem.ncbi.nlm.nih.gov/compound/Sodium-azide (accessed on 31 January 2020).

78. Ogata, M.; Zeng, X.; Usui, T.; Uzawa, H. Substrate specificity of $N$-acetylhexosaminidase from Aspergillus oryzae to artificial glycosyl acceptors having various substituents at the reducing ends. Carbohydr. Res. 2007, 342, 23-30. [CrossRef]

79. Uzawa, H.; Zeng, X.; Minoura, N. Synthesis of $6^{\prime}$-sulfodisaccharides by $\beta$ - $N$-acetylhexosaminidase-catalyzed transglycosylation. Chem. Commun. 2003, 100-101. [CrossRef] [PubMed] 
80. Crout, D.H.G.; Howarth, O.W.; Singh, S.; Swoboda, B.E.P.; Critchley, P.; Gibson, W.T. Biotransformations in carbohydrate synthesis. $N$-Acetylgalactosaminyl and $N$-acetylglucosaminyl transfer onto methyl $\alpha$ - and $\beta$-glucosides catalysed by the $\beta-N$-acetylhexosaminidase from Aspergillus oryzae. J. Chem. Soc. Chem. Commun. 1991, 1550-1551.

81. Crout, D.H.G.; Singh, S.; Swoboda, B.E.P.; Critchley, P.; Gibson, W.T. Biotransformations in carbohydrates synthesis. $N$-Acetylgalactosaminyl transfer on to methyl $N$-acetyl- $\beta$-D-glucosaminide (methyl 2-acetamido-2-deoxy- $\beta$-D-glucopyranoside) and methyl $N$-acetyl- $\alpha$-D-glucosaminide (methyl 2-acetamido-2-deoxy- $\alpha$-D-glucopyranoside). J. Chem. Soc. Chem. Commun. 1992, 53, 704-705. [CrossRef]

82. Singh, S.; Packwood, J.; Crout, D.H.G. Kinetic control of regioselectivity in glycosidase-catalysed disaccharide synthesis: Preparation of 2-acetamido-4-O-(2-acetamido2-deoxy- $\beta$-D-glucopyranosyl)-2-deoxy-D-glucopyranose ( $N, N^{\prime}$-diacetylchitobiose) and 2-acetamido-6-O(2-acetamido-2-deoxy- $\beta$-D-glucopyranosyl)-2-deoxy-D-glucopyranose. J. Chem. Soc. Chem. Commun. 1994, 2227-2228.

83. Křen, V.; Rajnochová, E.; Huňková, Z.; Dvořáková, J.; Sedmera, P. Unusual nonreducing sugar GlcNAc $\beta(1 \leftrightarrow 1)$ Man $\beta$ formation by $\beta-N$-acetylhexosaminidase from Aspergillus oryzae. Tetrahedron Lett. 1998, 39, 9777-9780. [CrossRef]

84. Singh, S.; Scigelova, M.; Vic, G.; Crout, D.H.G. Glycosidase-catalysed oligosaccharide synthesis of di-, triand tetra-saccharides using the $N$-acetylhexosaminidase from Aspergillus oryzae and the $\beta$-galactosidase from Bacillus circulans. J. Chem. Soc. Perkin Trans. 1 1996, 1921-1926. [CrossRef]

85. Drozdová, A.; Bojarová, P.; Křenek, K.; Weignerová, L.; Henßen, B.; Elling, L.; Christensen, H.; Jensen, H.H.; Pelantová, H.; Kuzma, M.; et al. Enzymatic synthesis of dimeric glycomimetic ligands of NK cell activation receptors. Carbohydr. Res. 2011, 346, 1599-1609. [CrossRef]

86. Hušáková, L.; Herkommerová-Rajnochová, E.; Semeňuk, T.; Kuzma, M.; Rauvolfová, J.; Přikrylová, V.; Ettrich, R.; Plíhal, O.; Bezouška, K.; Křen, V. Enzymatic Discrimination of 2-Acetamido-2-deoxy-D-mannopyranose-Containing Disaccharides Using $\beta$ - $N$-Acetylhexosaminidases. Adv. Synth. Catal. 2003, 345, 735-742. [CrossRef]

87. Weignerová, L.; Vavrušková, P.; Pišvejcová, A.; Thiem, J.; Křen, V. Fungal $\beta$-N-acetylhexosaminidases with high $\beta$-N-acetylgalactosaminidase activity and their use for synthesis of $\beta$-GalNAc-containing oligosaccharides. Carbohydr. Res. 2003, 338, 1003-1008. [CrossRef]

88. Bojarová, P.; Křenek, K.; Kuzma, M.; Petrásková, L.; Bezouška, K.; Namdjou, D.J.; Elling, L.; Křen, V. N-Acetylhexosamine triad in one molecule: Chemoenzymatic introduction of 2-acetamido-2-deoxy- $\beta$-D-galactopyranosyluronic acid residue into a complex oligosaccharide. J. Mol. Catal. B Enzym. 2008, 50, 69-73. [CrossRef]

89. Rauvolfová, J.; Kuzma, M.; Weignerová, L.; Fialová, P.; Přikrylová, V.; Pišvejcová, A.; Macková, M.; Křen, V. $\beta-N$-Acetylhexosaminidase-catalysed synthesis of non-reducing oligosaccharides. J. Mol. Catal. B Enzym. 2004, 29, 233-239. [CrossRef]

90. Aboitiz, N.; Cañada, F.J.; Hušáková, L.; Kuzma, M.; Křen, V.; Jiménez-Barbero, J. Enzymatic synthesis of complex glycosaminotrioses and study of their molecular recognition by herein domains. Org. Biomol. Chem. 2004, 2, 1987-1994. [CrossRef] [PubMed]

91. Hušáková, L.; Riva, S.; Casali, M.; Nicotra, S.; Kuzma, M.; Huňková, Z.; Křen, V. Enzymatic glycosylation using 6-O-acylated sugar donors and acceptors: $\beta-N$-acetylhexosaminidase-catalysed synthesis of 6-O,N,N'-triacetylchitobiose and 6'-O,N,N'-triacetylchitobiose. Carbohydr. Res. 2001, 331,143-148. [CrossRef]

92. Nekvasilová, P.; Andreasová, I.; Petrásková, L.; Pelantová, H.; Křen, V.; Bojarová, P. A novel enzymatic tool for transferring GalNAc moiety onto challenging acceptors. Biochim. Biophys. Acta Proteins Proteom. 2020, 1868, 140319. [CrossRef] [PubMed]

93. Fialová, P.; Namdjou, D.J.; Ettrich, R.; Přikrylová, V.; Rauvolfová, J.; Křenek, K.; Kuzma, M.; Elling, L.; Bezouška, K.; Křen, V. Combined application of galactose oxidase and $\beta-N$-acetylhexosaminidase in the synthesis of complex immunoactive N-acetyl-D-galactosaminides. Adv. Synth. Catal. 2005, 347, 997-1006. [CrossRef]

94. Garcia-Oliva, C.; Hoyos, P.; Petrásková, L.; Kulik, N.; Pelantová, H.; Cabanillas, A.H.; Rumbero, Á.; Křen, V.; Hernáiz, M.J.; Bojarová, P. Acceptor Specificity of $\beta$-N-Acetylhexosaminidase from Talaromyces flavus: A Rational Explanation. Int. J. Mol. Sci. 2019, 20, 6181. [CrossRef] 
95. Bojarová, P.; Kulik, N.; Hovorková, M.; Slámová, K.; Pelantová, H.; Křen, V. The $\beta$-N-acetylhexosaminidase in the synthesis of bioactive glycans: Protein and reaction engineering. Molecules 2019, 24, 599. [CrossRef]

96. Bojarová, P.; Kulik, N.; Slámová, K.; Hubálek, M.; Kotik, M.; Cvačka, J.; Pelantová, H.; Křen, V. Selective $\beta-N$-acetylhexosaminidase from Aspergillus versicolor-A tool for producing bioactive carbohydrates. Appl. Microbiol. Biotechnol. 2019, 103, 1737-1753. [CrossRef]

97. Bojarová, P.; Tavares, M.R.; Laaf, D.; Bumba, L.; Petrásková, L.; Konefał, R.; Bláhová, M.; Pelantová, H.; Elling, L.; Etrych, T.; et al. Biocompatible glyconanomaterials based on HPMA-copolymer for specific targeting of galectin-3. J. Nanobiotechnol. 2018, 16, 1-16.

98. Laaf, D.; Bojarová, P.; Mikulová, B.; Pelantová, H.; Křen, V.; Elling, L. Two-Step Enzymatic Synthesis of $\beta$-D-N-Acetylgalactosamine-( $\rightarrow 4$ )-D-N-acetylglucosamine (LacdiNAc) Chitooligomers for Deciphering Galectin Binding Behavior. Adv. Synth. Catal. 2017, 359, 2101-2108. [CrossRef]

99. Bojarová, P.; Chytil, P.; Mikulová, B.; Bumba, L.; Konefał, R.; Pelantová, H.; Krejzová, J.; Slámová, K.; Petrásková, L.; Kotrchová, L.; et al. Glycan-decorated HPMA copolymers as high-affinity lectin ligands. Polym. Chem. 2017, 8, 2647-2658. [CrossRef]

100. Nieder, V.; Kutzer, M.; Kren, V.; Gallego, R.G.; Kamerling, J.P.; Elling, L. Screening and characterization of $\beta-N$-acetylhexosaminidases for the synthesis of nucleotide-activated disaccharides. Enzym. Microb. Technol. 2004, 34, 407-414. [CrossRef]

101. Schmölzer, K.; Weingarten, M.; Baldenius, K.; Nidetzky, B. Glycosynthase Principle Transformed into Biocatalytic Process Technology: Lacto- $N$-triose II Production with Engineered exo-Hexosaminidase. ACS Catal. 2019, 9, 5503-5514. [CrossRef]

102. Teze, D.; Zhao, J.; Wiemann, M.; Ara, K.Z.G.; Lupo, R.; Rønne, M.; Carlström, G.; Duus, J.Ø.; Sanejouand, Y.-H.; O’Donohue, M.J.; et al. Rational Enzyme Design Without Structural Knowledge: A Sequence-Based Approach for Efficient Generation of Glycosylation Catalysts. ChemRxiv 2020. preprint. [CrossRef]

103. Chen, X.; Xu, L.; Jin, L.; Sun, B.; Gu, G.; Lu, L.; Xiao, M. Efficient and Regioselective Synthesis of $\beta$-GalNAc/GlcNAc-Lactose by a Bifunctional Transglycosylating $\beta$ - $N$-Acetylhexosaminidase from Bifidobacterium bifidum. Appl. Environ. Microbiol. 2016, 82, 5642-5652. [CrossRef] [PubMed]

104. Chen, X.; Jin, L.; Jiang, X.; Guo, L.; Gu, G.; Xu, L.; Lu, L.; Wang, F.; Xiao, M. Converting a $\beta$ - $N$-acetylhexosaminidase into two trans- $\beta-N$-acetylhexosaminidases by domain-targeted mutagenesis. Appl. Microbiol. Biotechnol. 2020, 104, 661-673. [CrossRef]

105. Schmölzer, K.; Weingarten, M.; Baldenius, K.; Nidetzky, B. Lacto-N-tetraose synthesis by wild-type and glycosynthase variants of the $\beta$-N-hexosaminidase from Bifidobacterium bifidum. Org. Biomol. Chem. 2019, 17, 5661-5665. [CrossRef]

106. Nilsson, K.G.I. Enzymic synthesis of di- and tri-saccharide glycosides, using glycosidases and $\beta$-D-galactoside 3- $\alpha$-sialyl-transferase. Carbohydr. Res. 1989, 188, 9-17. [CrossRef]

107. Biosynth Carbosynth, N,N'-Diacetylchitobiose. Available online: https://www.carbosynth.com/carbosynth/ website.nsf/(w-productdisplay)/9D1175C2DBD500A680256A6B0035991A (accessed on 31 January 2020).

108. Muschiol, J.; Meyer, A.S. A chemo-enzymatic approach for the synthesis of human milk oligosaccharide backbone structures. Z. Naturforsch. C J. Biosci. 2019, 74, 85-89. [CrossRef]

109. Nyffenegger, C.; Nordvang, R.T.; Zeuner, B.; Łężyk, M.; Difilippo, E.; Logtenberg, M.J.; Schols, H.A.; Meyer, A.S.; Mikkelsen, J.D. Backbone structures in human milk oligosaccharides: Trans-glycosylation by metagenomic $\beta-N$-acetylhexosaminidases. Appl. Microbiol. Biotechnol. 2015, 99, 7997-8009. [CrossRef]

110. White, T. 93. Studies in the amino-sugars. Part II. The action of dilute alkali solution on $N$-acylglucosamines. J. Chem. Soc. 1940, 428-437. [CrossRef]

111. Visnapuu, T.; Teze, D.; Kjeldsen, C.; Lie, A.; Duus, J.Ø.; André-Miral, C.; Pedersen, L.H.; Stougaard, P.; Svensson, B. Identification and Characterization of a $\beta$ - $N$-Acetylhexosaminidase with a Biosynthetic Activity from the Marine Bacterium Paraglaciecola hydrolytica S66T. Int. J. Mol. Sci. 2020, 21, 417. [CrossRef] [PubMed]

112. Biosynth Carbosynth, 2-Methyl-(1,2-dideoxy-a-D-glucopyrano)-[2,1-d]-2-Oxazoline. Available online: https:// www.carbosynth.com/carbosynth/website.nsf/(w-productdisplay)/A52FF54BFDD9F3508025775B0041E6FF (accessed on 31 January 2020).

113. Noguchi, M.; Tanaka, T.; Gyakushi, H.; Kobayashi, A.; Shoda, S.I. Efficient synthesis of sugar oxazolines from unprotected $\mathrm{N}$-acetyl-2-amino sugars by using chloroformamidinium reagent in water. J. Org. Chem. 2009, 74, 2210-2212. [CrossRef] [PubMed] 
114. Noguchi, M.; Fujieda, T.; Huang, W.C.; Ishihara, M.; Kobayashi, A.; Shoda, S.I. A practical one-step synthesis of 1,2-oxazoline derivatives from unprotected sugars and its application to chemoenzymatic $\beta-N$-acetylglucosaminidation of disialo-oligosaccharide. Helv. Chim. Acta 2012, 95, 1928-1936. [CrossRef]

115. Gill, I.; Valivety, R. Monosaccharide-Alkyl Glycoside Glass Phases: Plasticization with Hydrophilic and Hydrophobic Molecules. Angew. Chem. Int. Ed. Engl. 2000, 39, 3801-3804. [CrossRef]

116. Gill, I.; Valivety, R. Enzymatic Glycosylation in Plasticized Glass Phases: A Novel and Efficient Route to O-Glycosides. Angew. Chem. Int. Ed. Engl. 2000, 39, 3804-3808. [CrossRef]

117. Jamek, S.B.; Muschiol, J.; Holck, J.; Zeuner, B.; Busk, P.K.; Mikkelsen, J.D.; Meyer, A.S. Loop Protein Engineering for Improved Transglycosylation Activity of a $\beta$-N-Acetylhexosaminidase. ChemBioChem 2018, 19, 1858-1865. [CrossRef]

118. Murata, T.; Tashiro, A.; Itoh, T.; Usui, T. Enzymic synthesis of $3^{\prime}-O-$ and $6^{\prime}-\mathrm{O}-\mathrm{N}$-acetylglucosaminyl- $\mathrm{N}$-acetyllactosaminide glycosides catalyzed by $\beta$ - $N$-acetyl-D-hexosaminidase from Nocardia orientalis. Biochim. Biophys. Acta Gen. Subj. 1997, 1335, 326-334. [CrossRef]

119. Matahira, Y.; Tashiro, A.; Sato, T.; Kawagishi, H.; Usui, T. Enzymic synthesis of lacto-N-triose II and its positional analogues. Glycoconj. J. 1995, 12, 664-671. [CrossRef]

120. Murata, T.; Itoh, T.; Usui, T. Enzymatic synthesis of $\beta$-D-Gal-(1- >3)-[ $\beta$-D-GlcNAc- (1$>6$ )]- $\alpha$-D-GalNAc-OC6H4NO2-p as a carbohydrate unit of mucin-type 2 core. Glycoconj. J. 1998, 15, 575-582. [CrossRef]

121. Weignerová, L.; Suzuki, Y.; Huňková, Z.; Sedmera, P.; Havlíček, V.; Marek, R.; Křen, V. Pyridoxine as a Substrate for Screening Synthetic Potential of Glycosidases. Collect. Czechoslov. Chem. Commun. 1999, 64, 1325-1334. [CrossRef]

122. Kadowaki, S.; Saskiawan, I.; Watanabe, J.; Yamamoto, K.; Bunno, M.; Ichihara, Y.; Kumagai, H. Transglycosylation activity of $\beta-N$-acetylhexosaminidase from Penicillium oxalicum and its application to synthesis of a drug carrier. J. Ferment. Bioeng. 1997, 83, 341-345. [CrossRef]

123. Lundemo, P.; Karlsson, E.N.; Adlercreutz, P. Eliminating hydrolytic activity without affecting the transglycosylation of a GH1 $\beta$-glucosidase. Appl. Microbiol. Biotechnol. 2017, 101, 1121-1131. [CrossRef] [PubMed]

124. Lugaro, G.; Carrea, G.; Cremonesi, P.; Casellato, M.; Antonini, E. The Oxidation of Steroid Hormones by Fungal Laccase in Emulsion of Water and Organic Solvents. Arch. Biochem. Biophys. 1973, 159, 1-6. [CrossRef]

125. Bourquelot, E.; Bridel, M. Action de l'emulsine sur la gentiopicrine, en milieu alcoolique. J. Pharm. Chim. 1911, 4, 385-390.

126. Zaks, A.; Klibanov, A.M. Enzymatic Catalysis in Organic Media at $100{ }^{\circ}$ C. Science 1984, 224, $1249-1251$. [CrossRef] [PubMed]

127. Bornscheuer, U.; Schapöhler, S.; Scheper, T.; Schügerl, K. Influences of reaction conditions on the enantioselective transesterification using Pseudomonas cepacia lipase. Tetrahedron Asymmetry 1991, 2, 1011-1014. [CrossRef]

128. Cremonesi, P.; Casellato, M.M. Enzymic reactions in heterogeneous phase, preparation of $17 \alpha, 20 \beta$, 21-trihydroxypregn-4[14 C] en-3, 11-dione. J. Label. Compd. 1973, 9, 521-528. [CrossRef]

129. Bridiau, N.; Issaoui, N.; Maugard, T. The effects of organic solvents on the efficiency and regioselectivity of $N$-acetyl-lactosamine synthesis, using the $\beta$-galactosidase from Bacillus circulans in hydro-organic media. Biotechnol. Prog. 2010, 26, 1278-1289. [CrossRef]

130. Hansson, T.; Andersson, M.; Wehtje, E.; Adlercreutz, P. Influence of water activity on the competition between $\beta$-glycosidase-catalysed transglycosylation and hydrolysis in aqueous hexanol. Enzym. Microb. Technol. 2001, 29, 527-534. [CrossRef]

131. Lang, M.; Kamrat, T.; Nidetzky, B. Influence of ionic liquid cosolvent on transgalactosylation reactions catalyzed by thermostable $\beta$-glycosylhydrolase CelB from Pyrococcus furiosus. Biotechnol. Bioeng. 2006, 95, 1093-1100. [CrossRef] [PubMed]

132. Zeuner, B.; Riisager, A.; Mikkelsen, J.D.; Meyer, A.S. Improvement of trans-sialylation versus hydrolysis activity of an engineered sialidase from Trypanosoma rangeli by use of co-solvents. Biotechnol. Lett. 2014, 36, 1315-1320. [CrossRef] [PubMed]

133. Bayón, C.; Cortés, Á.; Berenguer, J.; Hernáiz, M.J. Highly efficient enzymatic synthesis of Gal $\beta-(1 \rightarrow$ 3)-GalNAc and Gal $\beta-(1 \rightarrow 3)-G l c N A c$ in ionic liquids. Tetrahedron 2013, 69, 4973-4978. [CrossRef] 
134. Kaftzik, N.; Wasserscheid, P.; Kragl, U. Use of Ionic Liquids to Increase the Yield and Enzyme Stability in the $\beta$-Galactosidase Catalysed Synthesis of N-Acetyllactosamine. Org. Process Res. Dev. 2002, 6, 553-557. [CrossRef]

135. Giacomini, C.; Irazoqui, G.; Gonzalez, P.; Batista-Viera, F.; Brena, B.M. Enzymatic synthesis of galactosyl-xylose by Aspergillus oryzae ß-galactosidase. J. Mol. Catal. B Enzym. 2002, 19-20, 159-165. [CrossRef]

136. Bayón, C.; Cortés, Á.; Aires-Trapote, A.; Civera, C.; Hernáiz, M.J. Highly efficient and regioselective enzymatic synthesis of $\beta-(1 \rightarrow 3)$ galactosides in biosolvents. RSC Adv. 2013, 3, 12155-12163. [CrossRef]

137. Slámová, K.; Bojarová, P. Engineered N-acetylhexosamine-active enzymes in glycoscience. Biochim. Biophys. Acta Gen. Subj. 2017, 1861, 2070-2087. [CrossRef]

138. Krieger, E.; Vriend, G. YASARA View-Molecular graphics for all devices-From smartphones to workstations. Bioinformatics 2014, 30, 2981-2982. [CrossRef]

139. Škerlová, J.; Bláha, J.; Pachl, P.; Hofbauerová, K.; Kukačka, Z.; Man, P.; Pompach, P.; Novák, P.; Otwinowski, Z.; Brynda, J.; et al. Crystal structure of native $\beta-N$-acetylhexosaminidase isolated from Aspergillus oryzae sheds light onto its substrate specificity, high stability, and regulation by propeptide. FEBS J. 2018, 285, 580-598. [CrossRef]

140. Straková, Z.; Slámová, K.; Kulik, N.; Křen, V. Transglycosidases engineered from the $\beta$-N-acetylhexosaminidase from Aspergillus oryzae (CBM13 Program Book, p. 149). Available online: https://cbm13.sciencesconf.org/data/pages/CBM13_Abstract_Book.pdf (accessed on 28 March 2020).

141. Mackenzie, L.F.; Wang, Q.; Warren, R.A.J.; Withers, S.G. Glycosynthases: Mutant Glycosidases for Oligosaccharide Synthesis. J. Am. Chem. Soc. 1998, 120, 5583-5584. [CrossRef]

142. Jahn, M.; Marles, J.; Warren, R.A.J.; Withers, S.G. Thioglycoligases: Mutant Glycosidases for Thioglycoside Synthesis. Angew. Chem. Int. Ed. Engl. 2003, 42, 352-354. [CrossRef] [PubMed]

143. Teze, D.; Hendrickx, J.; Czjzek, M.; Ropartz, D.; Sanejouand, Y.-H.; Tran, V.; Tellier, C.; Dion, M. Semi-rational approach for converting a GH1 $\beta$-glycosidase into a $\beta$-transglycosidase. Prot. Eng. Des. Sel. 2014, 27, 13-19. [CrossRef] [PubMed]

144. Teze, D.; Daligault, F.; Ferrières, V.; Sanejouand, Y.H.; Tellier, C. Semi-rational approach for converting a GH36 $\alpha$-glycosidase into an $\alpha$-transglycosidase. Glycobiology 2015, 25, 420-427. [CrossRef] [PubMed]

145. Bissaro, B.; Durand, J.; Biarnés, X.; Planas, A.; Monsan, P.; O’Donohue, M.J.; Fauré, R. Molecular Design of Non-Leloir Furanose-Transferring Enzymes from an $\alpha$-L-Arabinofuranosidase: A Rationale for the Engineering of Evolved Transglycosylases. ACS Catal. 2015, 5, 4598-4611. [CrossRef]

146. Yang, J.; Wang, Q.; Zhou, Y.; Li, J.; Gao, R.; Guo, Z. Engineering T. naphthophila ß-glucosidase for enhanced synthesis of galactooligosaccharides by site-directed mutagenesis. Biochem. Eng. J. 2017, 127, 1-8. [CrossRef]

147. Jers, C.; Michalak, M.; Larsen, D.M.; Kepp, K.P.; Li, H.; Guo, Y.; Kirpekar, F.; Meyer, A.S.; Mikkelsen, J.D. Rational Design of a New Trypanosoma rangeli Trans-Sialidase for Efficient Sialylation of Glycans. PLoS ONE 2014, 9, e83902. [CrossRef]

(C) 2020 by the authors. Licensee MDPI, Basel, Switzerland. This article is an open access article distributed under the terms and conditions of the Creative Commons Attribution (CC BY) license (http://creativecommons.org/licenses/by/4.0/). 